Prepared in cooperation with the University of Illinois, Prairie Research Institute, Illinois State Water Survey, NADP Program Office

\title{
External Quality-Assurance Project Report for the National Atmospheric Deposition Program/National Trends Network and Mercury Deposition Network, 2009-2010
}

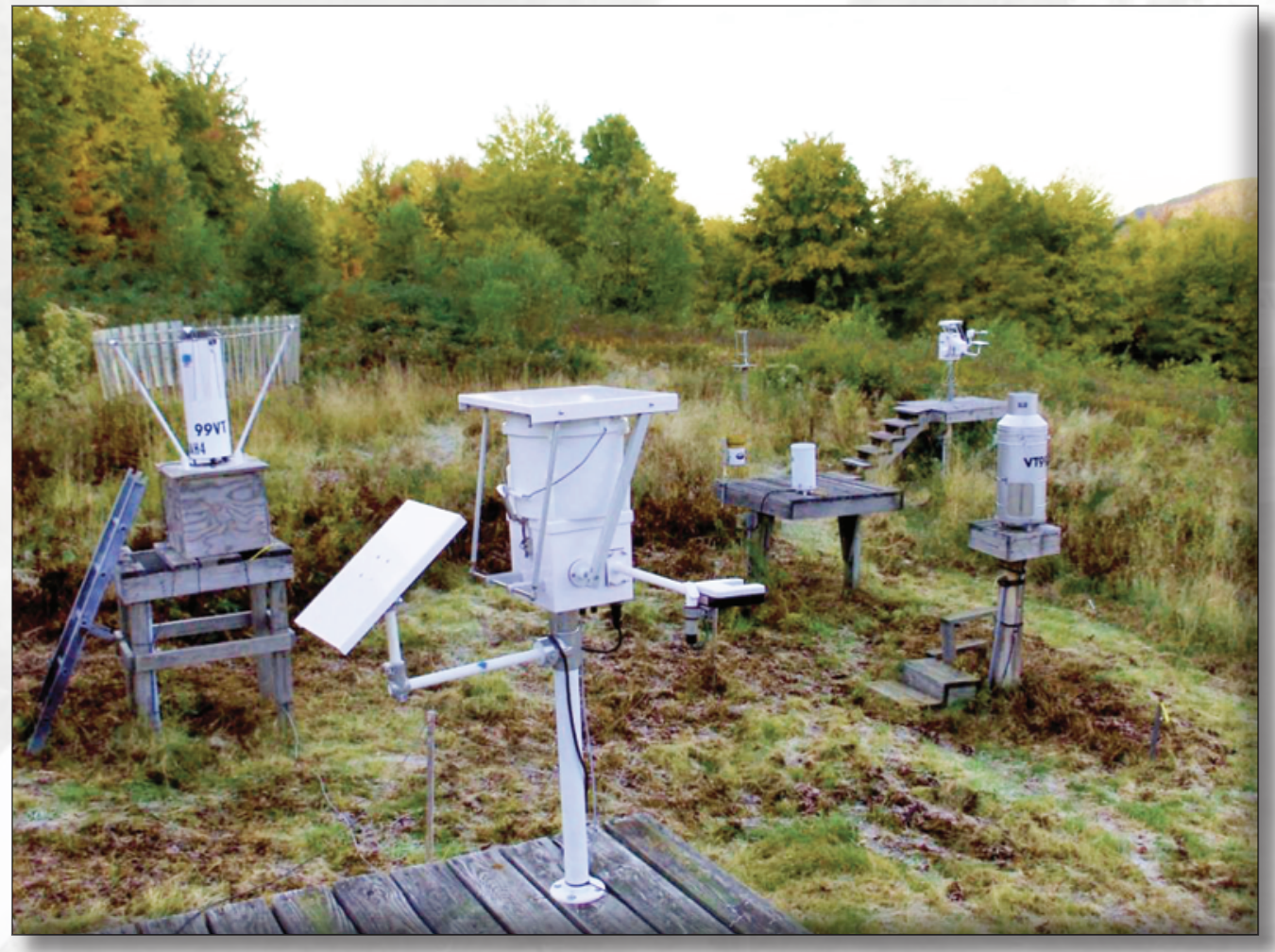

Scientific Investigations Report 2013-5147 



\section{External Quality-Assurance Project Report for the National Atmospheric Deposition Program/National Trends Network and Mercury Deposition Network, 2009-2010}

By Gregory A. Wetherbee, RoseAnn Martin, Mark F. Rhodes, and Tanya A. Chesney

Prepared in cooperation with the

University of Illinois, Prairie Research Institute, Illinois State Water Survey, NADP Program Office

Scientific Investigations Report 2013-5147 


\title{
U.S. Department of the Interior SALLY JEWELL, Secretary
}

\section{U.S. Geological Survey Suzette M. Kimball, Acting Director}

\author{
U.S. Geological Survey, Reston, Virginia: 2014
}

For more information on the USGS - the Federal source for science about the Earth, its natural and living resources, natural hazards, and the environment, visit http://www.usgs.gov or call 1-888-ASK-USGS.

For an overview of USGS information products, including maps, imagery, and publications, visit http://www.usgs.gov/pubprod

To order this and other USGS information products, visit http://store.usgs.gov

Any use of trade, firm, or product names is for descriptive purposes only and does not imply endorsement by the U.S. Government.

Although this information product, for the most part, is in the public domain, it also may contain copyrighted materials as noted in the text. Permission to reproduce copyrighted items must be secured from the copyright owner.

Suggested citation:

Wetherbee, G.A., Martin, RoseAnn, Rhodes, M.F., and Chesney, T.A., 2014, External quality-assurance project report for the National Atmospheric Deposition Program/National Trends Network and Mercury Deposition Network, 2009-2010: U.S. Geological Survey Scientific Investigations Report 2013-5147, 53 p.,

http://dx.doi.org/31.3133/sir20135147.

ISSN 2328-0328 (online) 


\section{Contents}

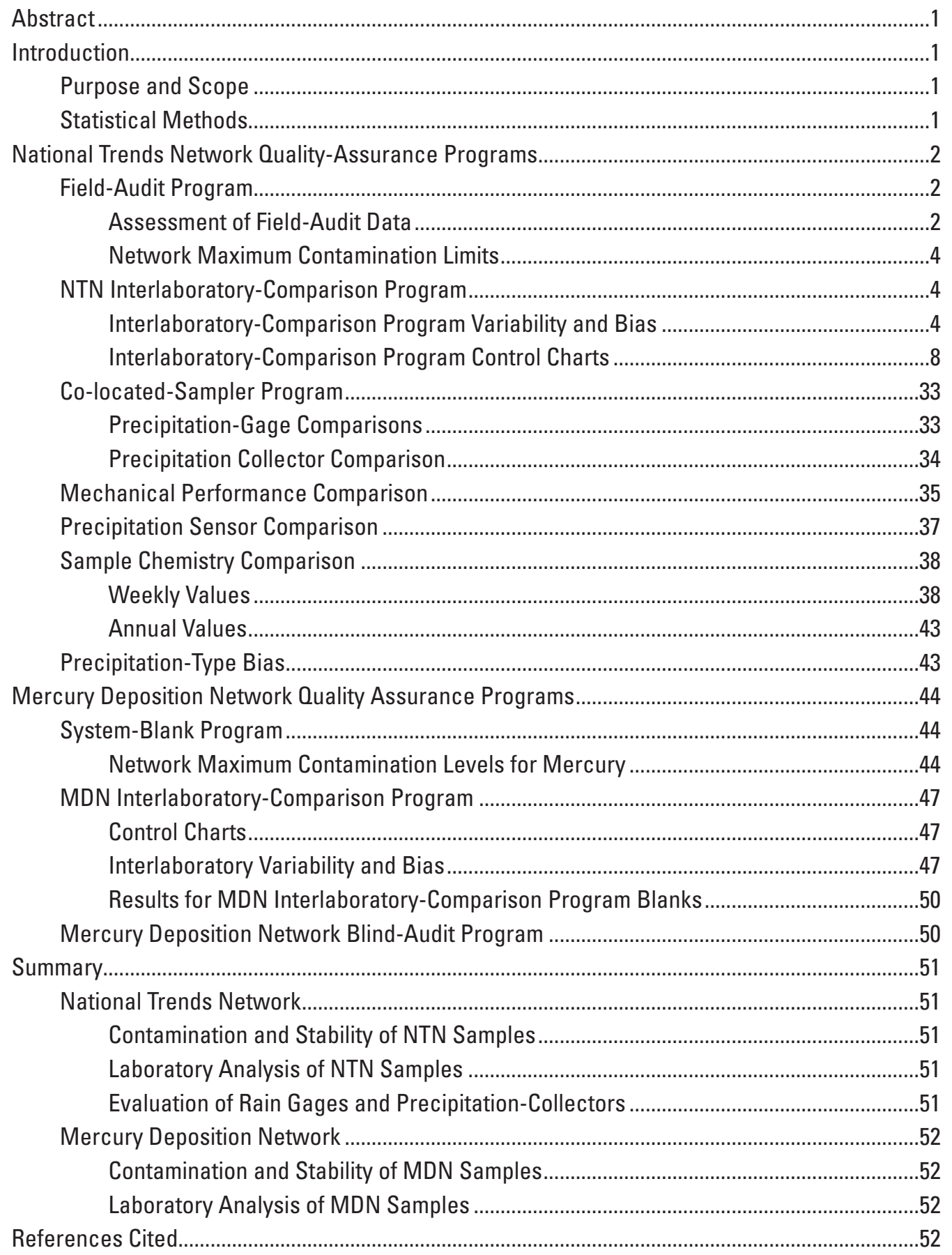




\section{Figures}

1. Three-year moving maximum contamination limits for National Trends Network analytes, 1997-2010. (NADP/NTN, National Atmospheric Deposition Program/National Trends Network)

2. Three-year moving maximum loss of ammonium, nitrate, and hydrogen ion from weekly National Trends Network samples 1997-2010. (NADP/NTN, National Atmospheric Deposition Program/National Trends Network; MDN, Mercury Deposition Network)

3. A, Difference between the measured calcium concentration values and the median calcium concentration value calculated by solution for all participating laboratories in the interlaboratory-comparison program during 2009-10 for MACTEC, Inc., Environment Canada Science and Technology Branch (ECST), Asia Center for Air and Precipitation Research (ACAP), and Central Analytical Laboratory (CAL) laboratories. $B$, Difference between the measured calcium concentration values and the median calcium concentration value calculated by solution for all participating laboratories in the interlaboratory-comparison program during 2009-10 for Norwegian Institute for Air Research (NILU), Shephard Analytical (SA), Ontario Ministry of Environment and Energy (MOEE), and New York

State Department of Environmental Conservation (NYSDEC) laboratories

4. A, Difference between the measured magnesium concentration values and the median magnesium concentration value calculated by solution for all participating laboratories in the interlaboratory-comparison program during 2009-10 for MACTEC, Inc., Environment Canada Science and Technology Branch (ECST), Asia Center for Air and Precipitation Research (ACAP), and Central Analytical Laboratory (CAL) laboratories. $B$, Difference between the measured magnesium concentration values and the median magnesium concentration value calculated by solution for all participating laboratories in the interlaboratory-comparison program during 2009-10 for Norwegian Institute for Air Research (NILU), Shephard Analytical (SA), Ontario Ministry of Environment and Energy (MOEE), and New York State Department of Environmental Conservation (NYSDEC) laboratories

5. A, Difference between the measured sodium concentration values and the median sodium concentration value calculated by solution for all participating laboratories in the interlaboratory-comparison program during 2009-10 for MACTEC, Inc., Environment Canada Science and Technology Branch (ECST), Asia Center for Air and Precipitation Research (ACAP), and Central Analytical Laboratory (CAL) laboratories. $B$, Difference between the measured sodium concentration values and the median sodium concentration value calculated by solution for all participating laboratories in the interlaboratory-comparison program during 2009-10 for Norwegian Institute for Air Research (NILU), Shephard Analytical (SA), Ontario Ministry of Environment and Energy (MOEE), and New York State Department of Environmental Conservation (NYSDEC) laboratories.. 
6. A, Difference between the measured potassium concentration values and the median potassium concentration value calculated by solution for all participating laboratories in the interlaboratory-comparison program during 2009-10 for MACTEC, Inc., Environment Canada Science and Technology Branch (ECST), Asia Center for Air and Precipitation Research (ACAP), and Central Analytical Laboratory (CAL) laboratories. $B$, Difference between the measured potassium concentration values and the median potassium concentration value calculated by solution for all participating laboratories in the interlaboratory-comparison program during 2009-10 for Norwegian Institute for Air Research (NILU), Shephard Analytical (SA), Ontario Ministry of Environment and Energy (MOEE), and New York State Department of Environmental Conservation (NYSDEC) laboratories.

7. A, Difference between the measured ammonium concentration values and the median ammonium concentration value calculated by solution for all participating laboratories in the interlaboratory-comparison program during 2009-10 for MACTEC, Inc., Environment Canada Science and Technology Branch (ECST), Asia Center for Air and Precipitation Research (ACAP), and Central Analytical Laboratory (CAL) laboratories. $B$, Difference between the measured ammonium concentration values and the median ammonium concentration value calculated by solution for all participating laboratories in the interlaboratory-comparison program during 2009-10 for Norwegian Institute for Air Research (NILU), Shephard Analytical (SA), Ontario Ministry of Environment and Energy (MOEE), and New York State Department of Environmental Conservation (NYSDEC) laboratories.

8. A, Difference between the measured chloride concentration values and the median chloride concentration value calculated by solution for all participating laboratories in the interlaboratory-comparison program during 2009-10 for MACTEC, Inc., Environment Canada Science and Technology Branch (ECST), Asia Center for Air and Precipitation Research (ACAP), and Central Analytical Laboratory (CAL) laboratories. $B$, Difference between the measured chloride concentration values and the median chloride concentration value calculated by solution for all participating laboratories in the interlaboratory-comparison program during 2009-10 for Norwegian Institute for Air Research (NILU), Shephard Analytical (SA), Ontario Ministry of Environment and Energy (MOEE), and New York State Department of Environmental Conservation (NYSDEC) laboratories

9. A, Difference between the measured nitrate concentration values and the median nitrate concentration value calculated by solution for all participating laboratories in the interlaboratory-comparison program during 2009-10 for MACTEC, Inc., Environment Canada Science and Technology Branch (ECST), Asia Center for Air and Precipitation Research (ACAP), and Central Analytical Laboratory (CAL) laboratories. $B$, Difference between the measured nitrate concentration values and the median nitrate concentration value calculated by solution for all participating laboratories in the interlaboratory-comparison program during 2009-10 for Norwegian Institute for Air Research (NILU), Shephard Analytical (SA), Ontario Ministry of Environment and Energy (MOEE), and New York State Department of Environmental Conservation (NYSDEC) laboratories. 
10. A, Difference between the measured sulfate concentration values and the median sulfate concentration value calculated by solution for all participating laboratories in the interlaboratory-comparison program during 2009-10 for MACTEC, Inc., Environment Canada Science and Technology Branch (ECST), Asia Center for Air and Precipitation Research (ACAP), and Central Analytical Laboratory (CAL) laboratories. $B$, Difference between the measured sulfate concentration values and the median sulfate concentration value calculated by solution for all participating laboratories in the interlaboratory-comparison program during 2009-10 for Norwegian Institute for Air Research (NILU), Shephard Analytical (SA), Ontario Ministry of Environment and Energy (MOEE), and New York State Department of Environmental Conservation (NYSDEC) laboratories..

11. A, Difference between the measured hydrogen-ion concentration values and the median hydrogen-ion concentration value calculated by solution for all participating laboratories in the interlaboratory-comparison program during 2009-10 for MACTEC, Inc., Environment Canada Science and Technology Branch (ECST), Asia Center for Air and Precipitation Research (ACAP), and Central Analytical Laboratory (CAL) laboratories. $B$, Difference between the measured hydrogen-ion concentration values and the median hydrogen-ion concentration value calculated by solution for all participating laboratories in the interlaboratory-comparison program during 2009-10 for Norwegian Institute for Air Research (NILU), Shephard Analytical (SA), Ontario Ministry of Environment and Energy (MOEE), and New York State Department of Environmental Conservation (NYSDEC) laboratories.

12. A, Difference between the measured specific conductance values and the median specific conductance value calculated by solution for all participating laboratories in the interlaboratory-comparison program during 2009-10 for MACTEC, Inc., Environment Canada Science and Technology Branch (ECST), Asia Center for Air and Precipitation Research (ACAP), and Central Analytical Laboratory (CAL) laboratories. $B$, Difference between the measured specific conductance values and the median specific conductance value calculated by solution for all participating laboratories in the interlaboratory-comparison program during 2009-10 for Norwegian Institute for Air Research (NILU), Shephard Analytical (SA), Ontario Ministry of Environment and Energy (MOEE), and New York State Department of Environmental Conservation (NYSDEC) laboratories..

13. Co-located N-CON collector 96IL and ACM collector IL11 (background-left) at Bondville, Illinois (top) and N-CON collector 99VT at Underhill, Vermont (bottom) ........32

14. Cumulative weekly precipitation depth during water years 2005-10 for paired ETI Noah-IV precipitation gages at co-located National Trends Network sites VT99 and 99VT, Underhill, Vermont.

15. Daily precipitation depth measurements for paired ETI Noah-IV precipitation gages at co-located National Trends Network sites C098/C089, Loch Vale, Colorado and VT99/99VT, Underhill, Vermont, water year 2010

16. Daily precipitation depth measurements at co-located Mercury Deposition Network sites IN26 and 26IN, Harrison State Park, Indiana, water year 2010 [ACM, Aerochem Metrics; MDN, Mercury Deposition Network]...

17. AeroChem Metrics (ACM) and N-CON NTN (N-CON) precipitation catch at co-located sites IL11/96IL and VT99/99VT during 2008-10

18. Aerochem Metrics-minus-N-CON weekly $A$, concentration and $B$, hydrogen-ion and specific-conductance differences for paired samples from co-located sites IL11/96IL and VT99/99VT during water years 2009-10.

19. Aerochem Metrics-minus-N-CON weekly sample-volume differences for paired samples from co-located sites IL11/96IL and VT99/99VT during water years 2009-10...42 
20. Weekly catch efficiency for co-located Aerochem Metrics (ACM) and N-CON precipitation collectors at IL11/96IL and VT99/99VT, water years 2009-10.

21. Upper confidence limits on mercury contamination concentration percentiles determined by 2009-10 system blank samples. (ng/L, nanograms per liter).....

22. A, Control charts for participating laboratories within the USGS interlaboratory-comparison program, Mercury Deposition Network, 2009-10. $B$, Control charts for USGS interlaboratory-comparison program for the Mercury Deposition Network, 2009-10.

\section{Tables}

1. Paired bucket-sample minus bottle-sample concentration differences for 2009-10 field-audit program

2. Maximum analyte contamination limits in 90 percent of 2009-10 field-audit samples and 2009-10 concentration quartiles for the National Atmospheric Deposition Program/National Trends Network

3. Most probable values for synthetic precipitation solutions used in 2009-10 U.S. Geological Survey interlaboratory-comparison program...

4. Median values for interlaboratory-comparison program natural precipitation samples estimated by the Kaplan Meir method and calculated by omission of censored values.

5. Differences between reported concentrations and most probable values for synthetic wet-deposition samples in the 2009 interlaboratory-comparison program ......9

6. Comparison of differences between reported concentrations and most probable values for synthetic wet-deposition samples in the 2010 interlaboratorycomparison program

7. Number of analyte determinations greater than the method detection limits in deionized-water samples for each participating laboratory and method detection limits for participating laboratories during 2009-10.

8. Precipitation depths measured at co-located rain gages; National Atmospheric Deposition Program sites VT99/99VT, C098/C089, IL11, and IN26/26IN, during water years 2005-10

9. Sample types for co-located AeroChem Metrics (ACM) and N-CON NTN (N-CON) collectors at sites VT99/99VT and IL11/96IL during water years 2009-2010.

10. Comparison of event data for co-located ACM and N-CON NTN precipitation collectors at co-located sites VT99/99VT during water years 2009-10.1.

11. Median weekly ACM-minus-N-CON concentration differences and results of sign test for bias between Aerochem Metrics and N-CON precipitation collectors

12. Annual precipitation-weighted mean concentration and deposition differences for co-located AeroChem Metrics and N-CON collectors at NADP sites VT99/99VT and IL11/96IL for weeks where data are available for both collectors during water year 2009

13. Results of Wilcoxon Signed Ranks test for precipitation-type bias in wet-deposition solute concentrations, specific conductance, and sample volume with respect to precipitation collector type and presence of frozen versus liquid precipitation.

14. Three-year moving average 90-percent upper confidence limits reported as network maximum contamination levels for total mercury $(\mathrm{Hg})$ concentration and mass estimated by system-blank samples, 2004-10.

15. Most probable values for solutions used during 2009-10 for the U.S. Geological Survey Mercury Deposition Network interlaboratory-comparison program 
16. Differences between reported mercury concentrations and most probable values for 2009-10 Mercury Deposition Network interlaboratory-comparison program samples

17. Mercury Deposition Network interlaboratory comparison program results for blank samples, 2009-10

\section{Abbreviations}

ACAP

ACM

ACMstd

ACMDB

ACZ

ADORC

AlRMoN

ALET

CAL

CALNAT

CVAFS

D00s

ECST

ETI Noah-IV

$f$-psig

FRL

HAL

$\mathrm{HCl}$

$\mathrm{Hg}$

HPS

IQR

ISWS

IVL

LRL

MAD

MACTEC

MAE

MDL

MDN
Asia Center for Air and Precipitation Research

AeroChem Metrics

Standard AeroChem Metrics Collector

AeroChem Metrics Deep Bucket Collector

ACZ Laboratories, Inc.

Acid Deposition and Oxidant Research Center

Atmospheric Integrated Research Monitoring Network

Atlantic Laboratory of Environmental Testing

Central Analytical Laboratory, Illinois State Water Survey

Natural precipitation samples

Cold vapor atomic fluorescence spectroscopy

Data quality objectives

Environment Canada Science and Technology Branch

Environmental Technologies, Inc. Noah-IV rain gage

$f$-pseudosigma

Flett Research, Limited

Mercury Analytical Laboratory, Frontier Global Sciences, Inc.

Hydrochloric acid

Mercury

High Purity Standards, Inc.

Interquartile range

Illinois State Water Survey

IVL-Swedish Environmental Institute

Laboratory reporting level

Median absolute difference, in units of concentration or microsiemens per centimeter

MACTEC, Inc.

Median absolute error, in percent

Method detection limit

Mercury Deposition Network 


$\begin{array}{ll}\text { MeHg } & \text { Methylmercury } \\ \text { MOEE } & \text { Ontario Ministry of Environment and Energy } \\ \text { MPV } & \text { Most probable value } \\ \text { MRL } & \text { Minimum reporting level } \\ \text { MSC } & \text { Meteorological Service of Canada } \\ \text { NADP } & \text { National Atmospheric Deposition Program } \\ \text { N-CON } & \text { N-CON Systems, Inc. } \\ \text { NTN } & \text { National Trends Network } \\ \text { NILU } & \text { Norwegian Institute for Air Research } \\ \text { NIST } & \text { National Institute of Standards and Technology } \\ \text { NLS } & \text { Northern Lake Service, Inc. } \\ \text { NMCL } & \text { Network maximum contamination limit } \\ \text { NOS } & \text { Network Operations Subcommittee } \\ \text { NSA } & \text { North Shore Analytical, Inc. } \\ \text { NYSDEC } & \text { New York State Department of Environmental Conservation } \\ \text { PCQA } & \text { U.S. Geological Survey Precipitation Chemistry Quality Assurance Project } \\ \text { OA } & \text { Ouality assurance } \\ \text { SA } & \text { Shepard Analytical } \\ \text { UCL } & \text { Upper confidence limit } \\ \text { USEPA } & \text { U.S. Environmental Protection Agency } \\ \text { USGS } & \text { U.S. Geological Survey } \\ \text { VITO } & \text { Flemish Institute of Technological Research } \\ \text { WML } & \text { U.S. Geological Survey, Wisconsin Mercury Laboratory } \\ & \end{array}$





\title{
U.S. Geological Survey External Quality-Assurance Project Report for the National Atmospheric Deposition Program/National Trends Network and Mercury Deposition Network, 2009-2010
}

\author{
By Gregory A. Wetherbee, RoseAnn Martin, Mark F. Rhodes, and Tanya A. Chesney
}

\section{Abstract}

The U.S. Geological Survey operated six distinct programs to provide external quality-assurance monitoring for the National Atmospheric Deposition Program/National Trends Network (NTN) and Mercury Deposition Network (MDN) during 2009-2010. The field-audit program assessed the effects of onsite exposure, sample handling, and shipping on the chemistry of NTN samples; a system-blank program assessed the same effects for MDN. Two interlaboratorycomparison programs assessed the bias and variability of the chemical analysis data from the Central Analytical Laboratory (CAL) and Mercury (Hg) Analytical Laboratory (HAL). The blind-audit program was also implemented for the MDN to evaluate analytical bias in total $\mathrm{Hg}$ concentration data produced by the HAL. The co-located-sampler program was used to identify and quantify potential shifts in NADP data resulting from replacement of original network instrumentation with new electronic recording rain gages (E-gages) and precipitation collectors that use optical sensors.

The results indicate that NADP data continue to be of sufficient quality for the analysis of spatial distributions and time trends of chemical constituents in wet deposition across the United States. Results also suggest that retrofit of the NADP networks with the new precipitation collectors could cause -8 to +14 percent shifts in NADP annual precipitationweighted mean concentrations and total deposition values for ammonium, nitrate, sulfate, and hydrogen ion, and larger shifts ( +13 to +74 percent) for calcium, magnesium, sodium, potassium, and chloride. The prototype N-CON Systems bucket collector is more efficient in the catch of precipitation in winter than Aerochem Metrics Model 301 collector, especially for light snowfall.

\section{Introduction}

Scientific investigators receive long-term, high-quality atmospheric wet-deposition information from the National Atmospheric Deposition Program (NADP) (Nilles, 2001).
Research scientists use NADP data to study the effects of atmospheric deposition on human health and the environment. Periodic evaluation of the NADP external quality-assurance results are intended to help investigators discern between true environmental signals and the variability and bias introduced by sample collection, processing, and laboratory analysis.

\section{Purpose and Scope}

This report presents independent quality assurance (QA) results obtained for 2009-10 (study period) for the U.S. Geological Survey (USGS) Precipitation Chemistry External Quality Assurance Project (PCQA) for the National Atmospheric Deposition Program (NADP). The project is administered by the USGS, Branch of Quality Systems (BQS), located in Denver, Colorado (Colo.). The NADP incorporates three wet-deposition monitoring networks: (1) National Trends Network (NTN), (2) Mercury Deposition Network (MDN), and (3) Atmospheric Integrated Research Monitoring Network (AIRMoN). AIRMoN data are not specifically addressed herein, but AIRMoN uses NTN monitoring protocols to collect event-based samples. Detailed information on the USGS QA procedures and analytical methods for NTN and MDN is available in Latysh and Wetherbee (2005 and 2007). Statistical methods used to evaluate the QA results are introduced herein and described in more detail by Gordon (1999), and Wetherbee and others (2004, 2005b, 2006, 2009, and 2010).

Most of the PCQA programs are operated on a calendaryear basis, but the co-located sampler program is operated on a water year (October 1 through September 30 of following year) basis. Four-character codes are used to identify NTN and MDN sites. Location information for the sites is available on the NADP web site at http://nadp.sws.uiuc.edu/. The two alpha characters represent the State in which the site is located; for example, AZ03 is site number 03 in Arizona.

\section{Statistical Methods}

In this report, nonparametric rank-based statistical methods are used in place of traditional statistics and hypothesis 
testing. Hypothesis tests were used to test for statistically significant differences between the central locations of different groups and included the Wilcoxon signed-rank test (Hollander and Wolfe, 1999) and the sign test (Kanji, 1993). Statistical analysis was performed using SAS version 9.2 software (SAS Institute Inc., 2001). Statistical tests were evaluated at the 95-percent level unless otherwise noted.

Bias was quantified by relative and absolute differences and percent differences and tested for significance using the sign test (Kanji, 1993). These techniques are discussed in previous external quality assurance reports for this project (Wetherbee and others, 2010). Variability is quantified in this report by the $f$-pseudosigma, a nonparametric analogue of the standard deviation of a statistical sample. The $f$-pseudosigma was calculated as the interquartile range (IQR) divided by 1.349 (Hoaglin and others, 1983):

$$
f \text {-pseudosigma }=\frac{75 \text { th percentile }-25 \text { th percentile }}{1.349}
$$

The $f$-pseudosigma ratio ( $f$-psig ratio) was used to compare an entire dataset's variability to a subset's variability, which is defined in equation 2 :

$$
f \text {-psig ratio }=\left(\frac{f p s i g_{\text {subset }}}{f p s i g_{o}}\right),
$$

where:

$$
\begin{array}{ll}
f_{p s i g} & =f \text {-pseudosigma of subset, and } \\
f_{\text {sig }} & =\text { overall } f \text {-pseudosigma of entire dataset. }
\end{array}
$$

An $f$-psig ratio less than 1 indicates less variability in the subset than overall, and an $f$-psig ratio greater than 1 indicates higher variability in the subset than overall. The $f$-psig can be expressed as a percentage by multiplying by 100 .

Data variability is evaluated herein to quantify precipitation-sample stability and contamination levels. Maximum contamination levels were determined by calculation of upper confidence limits (UCL) on percentiles of concentration data. Hahn and Meeker (1991) describe a method for determining a distribution-free UCL for a percentile using the binomial distribution, which is appropriate for environmental data that are commonly skewed.

The overall variability of NADP measurements is evaluated using co-located precipitation collectors and rain gages, which use similar field instruments to generate paired, replicate measurements of the same parameters at the same time and place. Measurement of the variability of NADP results is useful for verification of trends in NADP data. Conversely, co-located dissimilar precipitation collectors and(or) rain gages provide paired measurements to evaluate instrument bias. Instrument bias is evaluated herein for identification of potential shifts in trends that could result from changes in collector and rain-gage performance. These methods are discussed in more detail in Wetherbee and others (2005b, 2006, 2009, and 2010).

\section{National Trends Network Quality- Assurance Programs}

\section{Field-Audit Program}

The field-audit program is intended to identify changes to chemical concentrations in NTN wet-deposition samples that result from field exposure of the sample-collection apparatus. Field-audit samples are equipment rinse samples. A complete description of the program is given by Latysh and Wetherbee (2005) and Wetherbee and others (2010). Field-audit samples of deionized water or synthetic precipitation solutions were shipped to 100 sites each year of this study. As a standard part of site operation, operators poured 75 percent of the field-audit samples into their deployed wet-side buckets after a dry week of their choosing (bucket sample). The retained 25 percent of the solution in the original bottle is called the bottle sample. The bucket sample remains in the bucket for 24 hours, is then poured into a standard NTN sample bottle, and is shipped along with its paired bottle sample to the Central Analytical Laboratory (CAL) where both samples are analyzed.

A site that either submitted a sample to the CAL or a postcard to the USGS during the year was considered to have participated. Field-audit participation was 75 percent during 2009 and 70 percent during 2010 . Of the 75 sites participating in 2009, 67 pairs of bucket and bottle samples were submitted for analysis. Eight participating sites had no dry weeks. Of the 70 sites participating in 2010, 70 pairs of samples were submitted for analysis.

Of the 137 analyzed field-audit paired samples, 18 were processed in buckets containing traces of rinse water from the CAL cleaning process. Previous assessments have revealed no bias in chemistry due to residual CAL rinse water (Wetherbee and others 2005b, 2006, 2009, 2010).

\section{Assessment of Field-Audit Data}

Contamination may be introduced to NADP samples by dissolution of materials residing on the bucket walls. Alternatively, instability and loss of dissolved constituents from the solution could occur by adsorption to the bucket walls or other chemical or biological processes. Contamination and sample stability are evaluated by statistical analysis of paired bucketminus-bottle concentration differences for field-audit samples.

Before determining paired bucket-minus-bottle sample concentration differences for the field-audit data, concentrations less than the method detection limit (MDL) were set equal to one-half the MDL for computation of contamination concentrations. Only minor differences resulted from the treatment of less-than MDL values, such as substituting values reported as less-than MDLs with zero, with one-half the MDL, or with the MDLs themselves. Therefore, all of the values less than the MDL were set equal to one-half the MDL, which is 
a convenient substitution for purposes of capturing reasonable estimates of bias and variability using the non-parametric methods described earlier (Gibbons and Coleman, 2001). Helsel (2012) shows how such substitution leads to bias in hypothesis tests and calculation of statistical quantities, but for the purposes of this report, the substitution has little to no effect because the percentage of censored values is typically less than 25 percent and thus has no effect on quantification of the median and interquartile range.

A summary of paired bucket-minus-bottle concentration differences for the 2009-10 field-audit program data is given in table 1. Of the 137 sample pairs obtained in 2009-10, 99 (72 percent) had lower ammonium concentrations in the bucket samples than in the corresponding bottle samples, indicating loss of ammonium due to sample field exposure.
Ammonium can be lost from the samples by conversion to ammonia, which can volatilize, or via consumption by microorganisms (Fishman and others, 1986). However, the $f$-pseudosigma of ammonium concentration differences range from $0.006-0.009$ milligrams per liter $(\mathrm{mg} / \mathrm{L})$, which is in the range of the laboratory MDLs of $0.003-0.010 \mathrm{mg} / \mathrm{L}$ for $2009-10$. Therefore, differences in this concentration range might not be practically important. Of the 137 field-audit sample pairs analyzed during the study period, 88 (64 percent) had lower hydrogen-ion concentrations in the bucket samples than in the corresponding bottle samples. Neutralization can occur in many ways, such as sorption of hydrogen ion to the bucket, introduction of calcium and magnesium in dust when the lid seal is breached or when the lid opens during periods with no precipitation, and other processes.

Table 1. Paired bucket-sample minus bottle-sample concentration differences for 2009-10 field-audit program.

[Differences in milligrams per liter except hydrogen ion, in microequivalents per liter; and specific conductance, in microsiemens per centimeter at 25 degrees Celsius; $f$-pseudosigma was calculated as the interquartile range (IQR) divided by 1.349$]$

\begin{tabular}{|c|c|c|c|c|c|c|}
\hline \multirow[b]{3}{*}{ Analyte } & \multicolumn{6}{|c|}{ Paired bucket-minus-bottle differences } \\
\hline & \multirow[b]{2}{*}{ Minimum } & \multirow[b]{2}{*}{ Maximum } & \multicolumn{3}{|c|}{ Quartiles } & \multirow[b]{2}{*}{$f$-pseudosigma } \\
\hline & & & 25th & Median & 75th & \\
\hline \multicolumn{7}{|c|}{2009} \\
\hline Calcium & -0.051 & 0.192 & 0.002 & 0.005 & 0.01 & 0.006 \\
\hline Magnesium & -0.015 & 0.02 & 0 & 0.001 & 0.002 & 0.001 \\
\hline Sodium & -0.042 & 0.086 & 0 & 0.001 & 0.004 & 0.003 \\
\hline Potassium & -0.007 & 0.098 & 0 & 0 & 0.001 & 0.001 \\
\hline Ammonium & -0.061 & 0.022 & -0.012 & -0.004 & 0 & 0.009 \\
\hline Chloride & -0.044 & 0.116 & 0.001 & 0.004 & 0.008 & 0.005 \\
\hline Nitrate & -0.337 & 0.134 & 0 & 0.002 & 0.014 & 0.010 \\
\hline Sulfate & -0.313 & 0.136 & -0.003 & 0.001 & 0.014 & 0.013 \\
\hline Hydrogen ion & -11.34 & 2.847 & -1.081 & -0.378 & 0.069 & 0.852 \\
\hline Specific conductance & -3.2 & 1 & -0.2 & -0.1 & 0.1 & 0.222 \\
\hline \multicolumn{7}{|c|}{2010} \\
\hline Calcium & -0.021 & 0.555 & 0.002 & 0.006 & 0.012 & 0.007 \\
\hline Magnesium & -0.026 & 0.117 & -0.001 & 0 & 0.001 & 0.001 \\
\hline Sodium & -0.006 & 0.085 & 0 & 0.001 & 0.003 & 0.002 \\
\hline Potassium & -0.052 & 0.235 & -0.001 & 0 & 0.002 & 0.002 \\
\hline Ammonium & -0.124 & 1.395 & -0.004 & 0 & 0.004 & 0.006 \\
\hline Chloride & -0.015 & 0.119 & 0 & 0.003 & 0.008 & 0.006 \\
\hline Nitrate & -1.377 & 1.326 & -0.003 & 0 & 0.016 & 0.014 \\
\hline Sulfate & -0.031 & 2.289 & -0.001 & 0.001 & 0.007 & 0.006 \\
\hline Hydrogen ion & -24.48 & 6.698 & -1.561 & -0.524 & 0 & 1.157 \\
\hline Specific conductance & -8.3 & 3 & -0.6 & -0.2 & 0 & 0.445 \\
\hline
\end{tabular}




\section{Network Maximum Contamination Limits}

Statistical upper confidence limits (UCLs) for contamination percentiles provide an estimate of the amount of contamination that is not likely to be exceeded in a large percentage of NTN samples. Each year, the 90-percent UCL for the 90th percentile of field-audit paired concentration differences is considered the annual Network Maximum Contamination Limit (NMCL) for the NTN. The NMCL can be defined in several ways. First, the NMCL is the maximum contamination expected in 90 percent of the samples with 90-percent confidence. A second way of stating this is: There is a

10 -percent chance that contamination in the top 10 percent of NTN samples has been underestimated at the NMCL. A third way to express this is that there is 90-percent confidence that the contamination would exceed the NMCLs in 10 percent of the NTN samples. Finally, the NMCLs can serve as practical limits of quantitation for the network.

Estimated NMCLs for NTN analytes are compared to quartile values for all 2009-10 NTN data in table 2 (Christopher M.B. Lehmann, Central Analytical Laboratory, University of Illinois, written commun., 2012). During 2009, the NMCL for calcium was greater than the 25th percentile of all 2009 NADP/ NTN calcium concentrations, which suggests that the lower 25 percent of all calcium data during 2009 cannot be distinguished from sample contamination, but the 2010 NMCL for calcium was much lower. Likewise, 2009 NMCLs for magnesium, sodium, potassium, ammonium, and chloride are greater than or equal to the 25th percentile of all 2009 NTN values, suggesting that the lower 25 percent of 2009 NTN data for these constituents cannot be distinguished from sample contamination. Similar results were obtained for these constituents in 2010.

Results in figure 1 indicate that the 3 -year (yr) moving NMCLs were lower during 2008-10 than the previous 10 years for calcium, sodium, chloride, nitrate, and sulfate, but they have remained consistent for magnesium, potassium, and ammonium. The UCLs were also calculated for field audit bottle-minus-bucket concentration differences, to evaluate loss of ammonium, nitrate, and hydrogen ion, which are considered less stable than other NTN analytes. UCLs for analyte loss in figure 2 indicate that downward trends in ammonium, nitrate, and hydrogen-ion loss between 2000-08 were reversed in 2009 for unknown reasons.

Field-audit results for 2009-10 indicate that percentages of NTN sample concentrations less than the Network Maximum Contamination Limits (NMCLs) decreased from 2006-08 levels for all analytes except ammonium and nitrate (fig. 1). During 2008-10, ammonium and nitrate losses from NTN samples were greater than 2006-08 losses by approximately 0.40 and $0.01 \mathrm{mg} / \mathrm{L}$, respectively. Hydrogen-ion loss also increased slightly during 2008-10 (fig. 2).

\section{NTN Interlaboratory-Comparison Program}

The two objectives of the interlaboratory-comparison program are (1) to estimate the variability and bias of data reported by CAL and other participating laboratories and (2) to help facilitate integration of data from various wet-deposition monitoring networks without any attempt to account for the different onsite protocols used by different monitoring networks. Eight laboratories participated in the interlaboratory-comparison program during the study period: (1) Asia Center for Air and Precipitation Research (ACAP) in Niigata-shi, Japan; (2) Illinois State Water Survey CAL in Champaign, Illinois (Ill.); (3) MACTEC, Inc., in Gainesville, Florida; (4) Ontario Ministry of Environment and Energy, Dorset Research Facility (MOEE) in Dorset, Ontario, Canada; (5) Environment Canada Science and Technology Branch (ECST, formerly MSC) in Downsview, Ontario, Canada; (6) Norwegian Institute for Air Research (NILU) in Kjeller, Norway; (7) New York State Department of Environmental Conservation (NYSDEC) in Albany, New York; and (8) Shepard Analytical (SA) in Simi Valley, California. Many of the major global atmospheric-deposition monitoring networks are united into this single program designed to measure laboratory-data quality, which aids in data comparison between monitoring networks worldwide.

Each of the eight participating laboratories received four samples from USGS-BQS every month for chemical analysis, except for SA, which only received one-half of the samples. (SA dropped out of the program in April 2010 because it closed.) The three types of samples used in the interlaboratorycomparison program included: (1) synthetic precipitation samples prepared by USGS-BQS which are traceable to National Institute of Standards and Technology (NIST) reference materials (NIST-traceable samples); (2) deionized-water samples prepared by USGS-BQS; and (3) natural wet-deposition samples collected at NTN sites, blended by CAL, and sent to USGS-BQS for shipping to the laboratories as blind samples (Latysh and Wetherbee, 2005). Synthetic precipitation samples used in the interlaboratory-comparison program were made from stock solutions prepared by RT Corporation, Laramie, Wyoming for 2009, and by High Purity Standards (HPS), Charleston, South Carolina for 2010. Natural samples are filtered through 0.45 -micrometer $(\mathrm{mm})$ filters, bottled in 60- and 125-milliliter (mL) polyethylene bottles, and shipped in chilled, insulated containers to USGS at the Denver Federal Center to enhance stability of nutrient analytes: ammonium, nitrate, and sulfate in the samples (Tchobanoglous and Schroeder, 1987; and Wilde and others, 1998).

Median concentrations of each analyte were computed from the data submitted by the eight laboratories, which were considered to be the most probable values (MPVs) for each solution. Table 3 lists MPVs for the synthetic precipitation solutions and the number of samples analyzed per solution.

\section{Interlaboratory-Comparison Program Variability and Bias}

Censored concentration values reported as less than MDL were not included in MPV computations throughout the history of this program. However, the substitution of values for censored data can cause biased estimation of statistical 
Table 2. Maximum analyte contamination limits in 90 percent of 2009-10 field-audit samples and 2009-10 concentration quartiles for the National Atmospheric Deposition Program/National Trends Network.

[NADP/NTN, National Atmospheric Deposition Program/National Trends Network; Q1, 25th percentile; Q3, 75th percentile; all units in milligrams per liter (mg/L) except hydrogen ion in microequivalents per liter; na, not applicable]

\begin{tabular}{|c|c|c|c|c|c|c|c|c|c|c|c|c|}
\hline \multirow[b]{2}{*}{ Analyte } & \multicolumn{2}{|c|}{$\begin{array}{l}\text { Method detection limit } \\
\text { (MDL) } \\
\text { (mg/L) }\end{array}$} & \multicolumn{2}{|c|}{$\begin{array}{l}\text { Number and percent } \\
\text { censored field-audit } \\
\text { values less than MDL }\end{array}$} & \multicolumn{2}{|c|}{$\begin{array}{l}\text { Network Maximum } \\
\text { Contamination Limit } \\
\text { (NMCL) }\end{array}$} & \multicolumn{3}{|c|}{2009 NADP/NTN quartile values² } & \multicolumn{3}{|c|}{2010 NADP/NTN quartile values ${ }^{2}$} \\
\hline & 2009 & 2010 & 2009 & 2010 & 2009 & 2010 & 01 & Median & $\mathbf{0 3}$ & 01 & Median & 03 \\
\hline Calcium & 0.006 & 0.006 & $1(1 \%)$ & 0 & 0.069 & 0.038 & 0.050 & 0.109 & 0.245 & 0.047 & 0.103 & 0.225 \\
\hline Magnesium & 0.001 & 0.001 & $1(2 \%)$ & 0 & 0.009 & 0.010 & 0.009 & 0.020 & 0.045 & 0.001 & 0.003 & 0.007 \\
\hline Sodium & 0.001 & 0.001 & $1(1 \%)$ & 0 & 0.022 & 0.020 & 0.017 & 0.047 & 0.149 & 0.017 & 0.045 & 0.134 \\
\hline Potassium & 0.001 & 0.001 & $1(1 \%)$ & 0 & 0.023 & 0.033 & 0.009 & 0.018 & 0.036 & 0.009 & 0.017 & 0.035 \\
\hline Ammonium & 0.003 & 0.010 & $5(7 \%)$ & $15(21 \%)$ & 0.010 & 0.018 & 0.010 & 0.030 & 0.068 & 0.082 & 0.195 & 0.389 \\
\hline Chloride & 0.003 & 0.003 & $1(1 \%)$ & 0 & 0.045 & 0.040 & 0.009 & 0.020 & 0.045 & 0.040 & 0.083 & 0.225 \\
\hline Nitrate & 0.005 & 0.005 & $1(1 \%)$ & 0 & 0.048 & 0.064 & 0.418 & 0.730 & 1.196 & 0.382 & 0.706 & 1.189 \\
\hline Sulfate & 0.004 & 0.004 & $1(1 \%)$ & 0 & 0.136 & 0.033 & 0.392 & 0.723 & 1.238 & 0.310 & 0.624 & 1.094 \\
\hline Hydrogen ion & na & na & 0 & 0 & 1.240 & 1.520 & 2.570 & 7.586 & 15.488 & 2.754 & 7.244 & 14.791 \\
\hline
\end{tabular}

${ }^{1}$ Calculated as the 90-percent upper confidence limit for the 90th percentile of 2009 and 2010 field-audit bucket-minus-bottle paired differences using the binomial distribution function in SAS (SAS Institute, Inc., 2001).

${ }^{2}$ Data obtained from Christopher M.B. Lehmann, Central Analytical Laboratory, University of Illinois, written commun., 2012. 


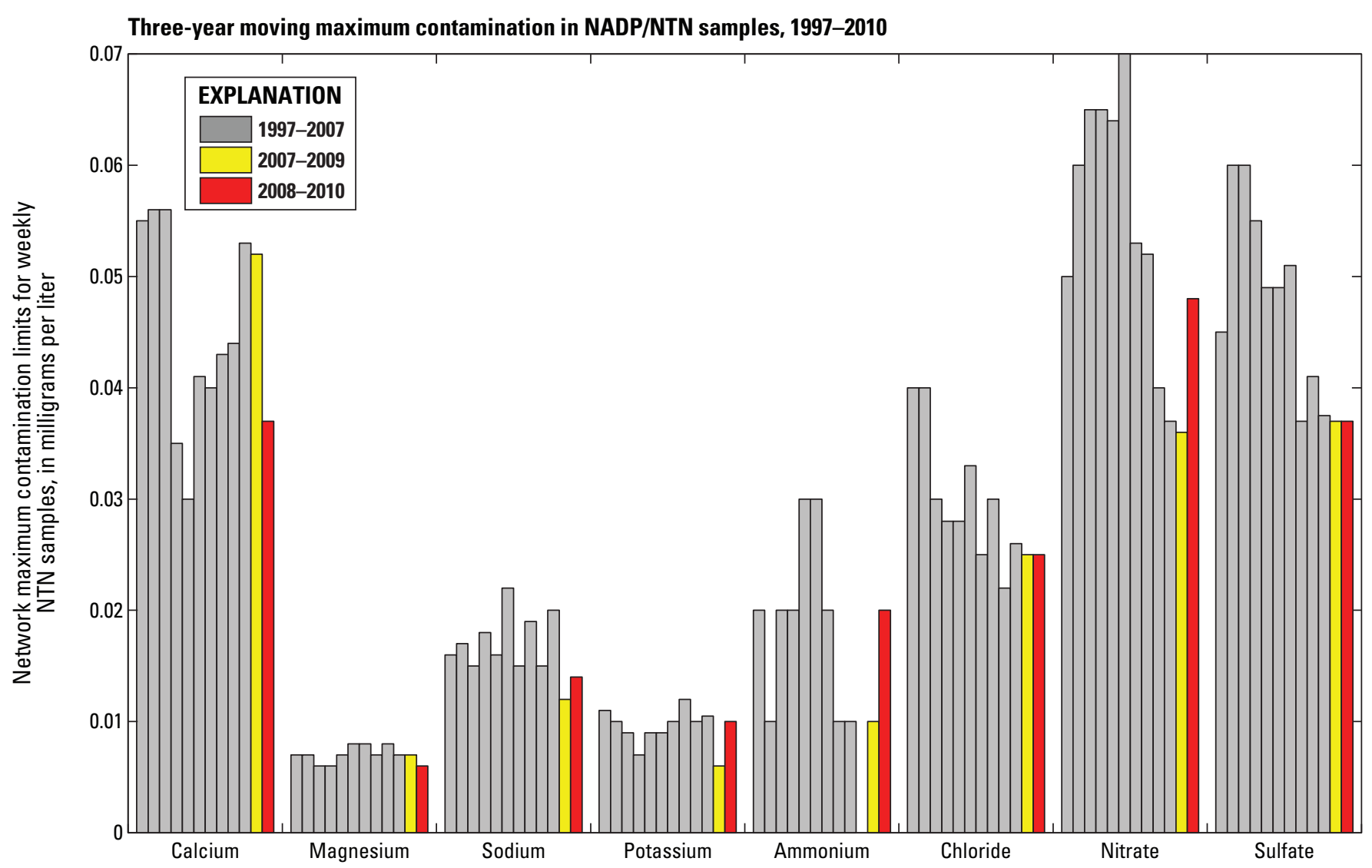

Figure 1. Three-year moving maximum contamination limits for National Trends Network analytes, 1997-2010. (NADP/NTN, National Atmospheric Deposition Program/National Trends Network)

parameters, such as the mean and median. A technique for appropriate use of censored values to estimate the mean or median is the Kaplan- Meir method, which is based on survival analysis (Helsel, 2012). The largest percentages of censored concentration values observed for this program are for magnesium and potassium, most commonly for natural matrix samples. Table 4 shows median values for natural precipitation samples estimated by the Kaplan-Meir methodology and values calculated by omitting missing values for censored data. Results in table 4 indicate that median values obtained by these two methods can be very different, especially for potassium concentrations. Percent differences in the median values obtained by these two estimation methods were calculated as:

where:

Difference in median estimations $=$

$$
\left.100 \times\left(\mathrm{M}_{\text {omit }}-\mathrm{M}_{\mathrm{KM}}\right) /\left(\left(\mathrm{M}_{\text {omit }}+\mathrm{M}_{\mathrm{KM}}\right) / 2\right)\right] \text {, }
$$

$$
\begin{aligned}
& \mathrm{M}_{\text {omit' }} \text { median obtained after censored values are } \\
& \text { omitted, and } \\
& \mathrm{M}_{\mathrm{KM}}, \quad \text { median obtained by the Kaplan Meir method. }
\end{aligned}
$$

All of the samples listed in table 4 are natural precipitation samples. There were no differences between the Kaplan Meir and substitution-method median values for synthetic precipitation samples (results not shown), which are traceable to NIST reference materials. Because the censoring frequency was found to be at least 25 percent for four analytes in 2 of 9 natural samples analyzed during 2009-10, the decision was made to use the Kaplan-Meir methodology for computation of the quartiles for the NTN interlaboratory-comparison program control charts for this report and for subsequent reports.

Censored concentrations were set to one-half the MDL for calculation of differences from the MPVs for each laboratory. This substitution does not affect the sign test when less than 50 percent of the values are censored, and it does not affect calculation of the $f$-psig ${ }_{0}$ and $f$-psig ${ }_{\text {lab }}$ calculations when less than 25 percent of the values are censored, which is true for the results from all eight laboratories. During 2009-10, CAL reported no values less than the MDL for both natural and synthetic precipitation samples.

Interlaboratory bias for the participating laboratories was evaluated by the following methods: (1) Comparison of the medians of the differences between laboratory results and MPVs, (2) hypothesis testing using the sign test (Kanji, 1993), and (3) comparison of laboratory results for deionizedwater samples. The arithmetic signs of the median differences indicate whether the reported results for each constituent are 
Three-year moving maximum loss in NADP/NTN samples, 1997-2010

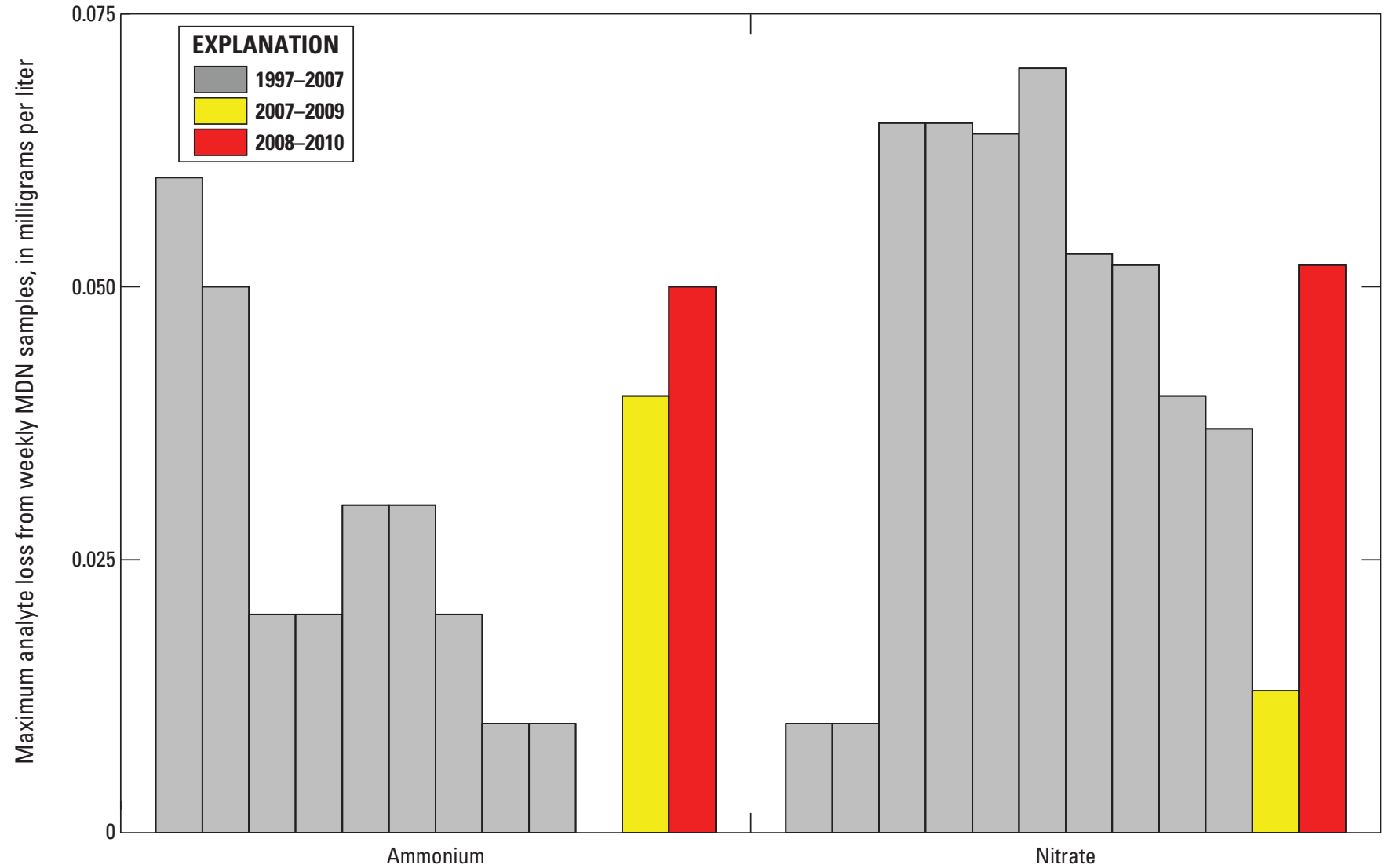

Figure 2. Three-year moving maximum loss of ammonium, nitrate, and hydrogen ion from weekly National Trends Network samples 1997-2010. (NADP/NTN, National Atmospheric Deposition Program/National Trends Network; MDN, Mercury Deposition Network)

Table 3. Most probable values for synthetic precipitation solutions used in 2009-10 U.S. Geological Survey interlaboratorycomparison program.

$\left[\mathrm{Ca}^{2+}\right.$, calcium; $\mathrm{Mg}^{2+}$, magnesium; $\mathrm{Na}^{+}$, sodium; $\mathrm{K}^{+}$, potassium; $\mathrm{NH}_{4}^{+}$, ammonium; $\mathrm{Cl}^{-}$, chloride; $\mathrm{NO}_{3}^{-}$, nitrate; $\mathrm{SO}_{4}^{2-}$, sulfate; $\mathrm{H}^{+}$, hydrogen ion; all units in milligrams per liter except hydrogen ion, in microequivalents per liter and specific conductance, in microsiemens per centimeter at 25 degrees Celsius; na, not applicable]

\begin{tabular}{|c|c|c|c|c|c|c|c|c|c|c|c|}
\hline Solution & $\mathrm{Ca}^{2+}$ & $\mathbf{M g}^{2+}$ & $\mathrm{Na}^{+}$ & $\mathbf{K}^{+}$ & $\mathbf{N H}_{4}^{+}$ & $\mathrm{Cl}^{-}$ & $\mathrm{NO}_{3}^{-}$ & $\mathrm{SO}_{4}{ }^{2-}$ & $\mathrm{H}^{+}$ & $\begin{array}{c}\text { Specific } \\
\text { conductance }\end{array}$ & $\begin{array}{c}\text { Number of } \\
\text { samples shipped } \\
\text { and analyzed pe } \\
\text { laboratory }\end{array}$ \\
\hline & \multicolumn{11}{|c|}{2009} \\
\hline SP1 & 0.391 & 0.086 & 0.403 & 0.078 & 0.435 & 0.540 & 3.397 & 3.6375 & 26.516 & 31.2 & 4 \\
\hline SP17 & 0.051 & 0.011 & 0.047 & 0.009 & 0.055 & 0.064 & 0.417 & 0.450 & 5.370 & 4.69 & 4 \\
\hline SP21 & 0.206 & 0.033 & 0.169 & 0.027 & 0.178 & 0.190 & 2.099 & 1.120 & 7.762 & 12.14 & 4 \\
\hline SP3 & 0.140 & 0.046 & 0.104 & 0.031 & 0.089 & 0.152 & 1.496 & 0.912 & 28.840 & 18.35 & 4 \\
\hline \multirow[t]{2}{*}{ SP97 } & 0.112 & 0.018 & 0.022 & 0.019 & 0.184 & 0.050 & 1.116 & 1.096 & 28.708 & 18.1 & 4 \\
\hline & \multicolumn{11}{|c|}{2010} \\
\hline SP1 & 0.440 & 0.089 & 0.398 & 0.073 & 0.664 & 0.579 & 2.041 & 3.700 & 36.308 & 28.1 & 5 \\
\hline SP21 & 0.216 & 0.034 & 0.170 & 0.028 & 0.270 & 0.212 & 1.442 & 1.116 & 14.454 & 11.6 & 5 \\
\hline SP3 & 0.152 & 0.046 & 0.104 & 0.022 & 0.132 & 0.164 & 1.046 & 0.938 & 14.962 & 10.1 & 5 \\
\hline SP97 & 0.124 & 0.018 & 0.021 & 0.018 & 0.280 & 0.050 & 1.124 & 1.080 & 16.788 & 11.2 & 5 \\
\hline
\end{tabular}


Table 4. Median values for interlaboratory-comparison program natural precipitation samples estimated by the Kaplan Meir method and calculated by omission of censored values.

$\left[\mathrm{N}\right.$, number of samples; $\mathrm{M}_{\mathrm{KM}}$, median estimated by Kaplan-Meir method; $\mathrm{M}_{\text {omit }}$, median calculated after omitting censored values; $\mathrm{Ca}^{2+}$, calcium; $\mathrm{Mg}^{2+}$, magnesium; $\mathrm{Na}^{+}$, sodium; $\mathrm{K}^{+}$, potassium; $\mathrm{NH}_{4}^{+}$, ammonium; $\mathrm{Cl}$, chloride; $\mathrm{NO}_{3}^{-}$, nitrate; $\mathrm{SO}_{4}^{2-}$, sulfate; all units in milligrams per liter; natural wet-deposition samples blended and shipped to USGS Branch of Quality Systems in Denver, Colo. by the Illinois State Water Survey, Central Analytical Laboratory]

\begin{tabular}{|c|c|c|c|c|c|c|}
\hline $\begin{array}{c}\text { Sample } \\
\text { ID }\end{array}$ & Analyte & $\mathbf{N}$ & $\begin{array}{l}\text { Censoring } \\
\text { frequency } \\
\text { (percent) }\end{array}$ & МКМ & Momit & $\begin{array}{l}\text { Difference } \\
\text { in median } \\
\text { estimations } \\
\text { (percent) }\end{array}$ \\
\hline \multirow[t]{2}{*}{ TF741 } & $\mathrm{Mg}^{2+}$ & 19 & 32 & 0.003 & 0.024 & 156 \\
\hline & $\mathrm{K}^{+}$ & 21 & 25 & 0.018 & 0.023 & 24 \\
\hline TF959 & $\mathrm{K}^{+}$ & 26 & 7 & 0.018 & 0.020 & 16 \\
\hline TG188 & $\mathrm{K}^{+}$ & 26 & 7 & 0.024 & 0.028 & 16 \\
\hline \multirow[t]{5}{*}{ TG625 } & $\mathrm{Ca}^{2+}$ & 22 & 21 & 0.023 & 0.045 & 65 \\
\hline & $\mathrm{Mg}^{2+}$ & 24 & 14 & 0.014 & 0.020 & 36 \\
\hline & $\mathrm{Na}^{+}$ & 23 & 18 & 0.017 & 0.028 & 49 \\
\hline & $\mathrm{K}^{+}$ & 23 & 18 & 0.035 & 0.062 & 56 \\
\hline & $\mathrm{Cl}^{-}$ & 24 & 14 & 0.022 & 0.026 & 21 \\
\hline \multirow[t]{3}{*}{ ТH023 } & $\mathrm{Ca}^{2+}$ & 22 & 21 & 0.010 & 0.024 & 81 \\
\hline & $\mathrm{Mg}^{2+}$ & 22 & 21 & 0.041 & 0.080 & 64 \\
\hline & $\mathrm{K}^{+}$ & 25 & 11 & 0.019 & 0.020 & 5 \\
\hline \multirow[t]{2}{*}{ TH429 } & $\mathrm{NH}_{4}^{+}$ & 12 & 14 & 0.355 & 0.356 & 0 \\
\hline & $\mathrm{NO}_{3}^{-}$ & 12 & 14 & 0.604 & 0.608 & 0 \\
\hline TH650 & $\mathrm{Na}^{+}$ & 26 & 7 & 0.014 & 0.015 & 7 \\
\hline TH894 & $\mathrm{K}^{+}$ & 13 & 7 & 0.020 & 0.021 & 2 \\
\hline \multirow[t]{4}{*}{ TI098 } & $\mathrm{Ca}^{2+}$ & 26 & 7 & 0.021 & 0.022 & 5 \\
\hline & $\mathrm{Mg}^{2+}$ & 17 & 39 & 0.006 & 0.010 & 50 \\
\hline & $\mathrm{K}^{+}$ & 18 & 36 & 0.010 & 0.012 & 18 \\
\hline & $\mathrm{SO}_{4}^{2-}$ & 27 & 4 & 0.200 & 0.210 & 5 \\
\hline
\end{tabular}

positively or negatively biased. The sign test null hypothesis is: "The true median of the reported-minus-MPV differences is zero." The test results were evaluated at the alpha $(\alpha)=0.05$ significance level for a two-tailed test.

Variability between laboratories was compared using the $f$-psig ratios (equation 2). Tables 5 and 6 show results for evaluating variability and bias (analogous to precision and accuracy) of the analytical data for each of the laboratories participating in the 2009-10 interlaboratory-comparison program. Shaded values in tables 5 and 6 identify analytes for which both (1) a statistically significant bias $(\alpha=0.05)$ was estimated by the sign test, and (2) the absolute value of the median relative concentration difference for the analytes was greater than the participant's analytical detection limit. Sodium and sulfate concentrations were slightly biased high, and nitrate concentrations were slightly biased low for CAL during 2009, but no bias was indicated during 2010. Data submitted by the CAL had the lowest overall variability of the eight participating laboratories during 2009-10. CAL results indicated no detections of analytes greater than the MDLs for four deionized water blanks each year during 2009-10 (table 7).

\section{Interlaboratory-Comparison Program Control Charts}

The results from each participating laboratory are compared to the MPVs over time in the control charts shown in figures $3 A-12 B$. Points in the control charts are color- and symbol-coded by solution type to provide a visual indication of potential bias for specific solutions. Of the concentration differences that exceeded the control limits, most tended to be for filtered, natural precipitation (CALNAT) samples, which are more variable in composition than the synthetic precipitation sample made from reagents.

Control charts for CAL show more values outside the statistical control limits than typically observed in the recent past, especially for calcium, magnesium, sodium, and potassium results for natural precipitation samples (Wetherbee and others, 2010, 2009, 2006, 2004). Because previously used censored-value substitution techniques have been shown to bias computation of statistical parameters (Helsel, 2012), the PCQA project began calculation of quartiles for control charts using the Kaplan-Meir technique for treatment of censored data starting with this report. Therefore, changes in frequency of values exceeding the control limits could be due to this change to the Kaplan-Meir methodology. Despite this change, CAL data were within statistical control during at least 90 percent of the study period. CAL precision was consistent with that of MACTEC, ECST, and SA for most constituents.

Variability and bias are illustrated in the control charts for other selected participating laboratories as well. High variability among all of the participating laboratories for ammonium and nitrate results is evident. Chloride variability was high among all participating laboratories during the same period. 
Table 5. Differences between reported concentrations and most probable values for synthetic wet-deposition samples in the 2009 interlaboratory-comparison program.

$[\%$, percent; sign test $p$-value, probability of rejecting the null hypothesis: The true median of the differences between laboratory results and the most probable value is zero, when true; values are shaded where the bias is greater than the method detection limit and is statistically significant ( $\alpha=0.05)$ (Kanji, 1993); Spec. cond., specific conductance; ACAP, Asia Center for Air and Precipitation Reasearch; CAL, Central Analytical Laboratory, Illinois State Water Survey; MACTEC, MACTEC, Inc.; MOEE, Ontario Ministry of Environment and Energy; ECST, Environment Canada Science and Technology Branch; NILU, Norwegian Institute for Air Research; NYSDEC, New York State Department of Environmental Conservation; SA, Shepard Analytical; --, not calculated; <, less than]

\begin{tabular}{|c|c|c|c|c|c|c|c|c|c|c|c|c|c|}
\hline \multirow[b]{3}{*}{ Analyte } & \multirow[b]{3}{*}{$\begin{array}{c}\text { Overall } \\
\text { f-psig }^{4} \\
\text { (units }^{1,2,3} \text { ) }\end{array}$} & \multicolumn{12}{|c|}{ Laboratory } \\
\hline & & \multicolumn{3}{|c|}{ ACAP } & \multicolumn{3}{|c|}{ CAL } & \multicolumn{3}{|c|}{ MACTEC } & \multicolumn{3}{|c|}{ MOEE } \\
\hline & & $\begin{array}{c}\text { Median } \\
\text { diff. }^{5} \\
\text { (units }^{1,2,3} \text { ) }\end{array}$ & $\begin{array}{c}\text { sign } \\
\text { test } \\
p \text {-value }\end{array}$ & $\begin{array}{c}f \text {-psig } \\
\text { ratio }^{6} \\
(\%)\end{array}$ & $\begin{array}{c}\text { Median } \\
\text { diff. }^{5} \\
\text { (units }^{1,2,3} \text { ) }\end{array}$ & $\begin{array}{c}\text { sign } \\
\text { test } \\
p \text {-value }\end{array}$ & $\begin{array}{c}f \text {-psig } \\
\text { ratio }^{6} \\
(\%)\end{array}$ & $\begin{array}{c}\text { Median } \\
\text { diff. }^{5} \\
\text { (units }^{1,2,3} \text { ) }\end{array}$ & $\begin{array}{c}\text { sign } \\
\text { test } \\
p \text {-value }\end{array}$ & $\begin{array}{c}f \text {-psig } \\
\text { ratio }^{6} \\
(\%)\end{array}$ & $\begin{array}{c}\text { Median } \\
\text { diff. }^{5} \\
\text { (units }^{1,2,3} \text { ) }\end{array}$ & $\begin{array}{c}\text { sign } \\
\text { test } \\
p \text {-value }\end{array}$ & $\begin{array}{r}f-p s i g \\
\text { ratio } \\
(\%)\end{array}$ \\
\hline Calcium $^{1}$ & 0.011 & 0.005 & 0.1153 & 460 & 0.001 & 0.8145 & 22 & 0.001 & 0.5034 & 54 & 0.011 & 0.6476 & 67 \\
\hline Magnesium $^{1}$ & 0.003 & -0.002 & 0.0042 & 91 & 0.001 & $<0.0001$ & 33 & 0.000 & 0.0574 & 34 & 0.002 & 0.3593 & 193 \\
\hline Sodium $^{1}$ & 0.004 & 0.000 & 0.2379 & 90 & 0.003 & 0.0003 & 50 & 0.002 & 0.0636 & 57 & 0.002 & 1.0000 & 250 \\
\hline Potassium ${ }^{1}$ & 0.004 & 0.002 & 0.0007 & 44 & 0.001 & 0.0005 & 17 & 0.001 & 0.0574 & 20 & -0.007 & 0.6476 & 105 \\
\hline Ammonium $^{1}$ & 0.010 & 0.077 & $<0.0001$ & 1404 & 0.003 & $<0.0001$ & 35 & -0.001 & 1.0000 & 25 & 0.097 & 0.0003 & 54 \\
\hline Chloride $^{1}$ & 0.007 & 0.006 & 0.6476 & 148 & 0.002 & 0.0192 & 23 & -0.001 & 0.8036 & 48 & 0.010 & 0.3018 & 395 \\
\hline Nitrate $^{1}$ & 0.040 & -0.061 & 0.8238 & 433 & -0.018 & $<0.0001$ & 25 & 0.000 & 0.2632 & 66 & -0.482 & 0.0023 & 34 \\
\hline Sulfate $^{1}$ & 0.036 & 0.001 & 0.3323 & 113 & 0.014 & 0.0007 & 36 & -0.002 & 0.8145 & 29 & 0.038 & 0.0213 & 204 \\
\hline Hydrogen ion ${ }^{2}$ & 2.922 & -10.940 & 0.5034 & 178 & 0.000 & 0.8238 & 69 & -1.822 & 0.4514 & 32 & -10.925 & 0.0213 & 92 \\
\hline \multirow[t]{2}{*}{ Spec. cond ${ }^{3}$} & 1.030 & -3.385 & 0.0118 & 1150 & 0.000 & 1.0000 & 31 & 0.975 & 0.0118 & 53 & -8.5 & $<0.0001$ & 68 \\
\hline & & \multicolumn{3}{|c|}{ ECST } & \multicolumn{3}{|c|}{ NILU } & \multicolumn{3}{|c|}{ NYSDEC } & \multicolumn{3}{|c|}{ SA } \\
\hline Calcium $^{1}$ & 0.011 & 0.001 & 0.4807 & 51 & -0.002 & 0.2632 & 81 & -0.033 & $<0.0001$ & 152 & 0.003 & 0.8238 & 66 \\
\hline Magnesium $^{1}$ & 0.003 & 0.000 & 0.0129 & 16 & -0.003 & 0.1153 & 99 & -0.008 & $<0.0001$ & 179 & 0.001 & 1.0000 & 69 \\
\hline Sodium ${ }^{1}$ & 0.004 & -0.001 & 0.0192 & 70 & 0.000 & 0.5034 & 325 & -0.007 & 0.0026 & 170 & 0.000 & 0.3877 & 15 \\
\hline Potassium ${ }^{1}$ & 0.004 & 0.001 & 0.1153 & 190 & -0.001 & 0.1153 & 58 & -0.006 & $<0.0001$ & 65 & -0.003 & $<0.0001$ & 93 \\
\hline Ammonium $^{1}$ & 0.010 & -0.002 & 1.0000 & 31 & -0.025 & 0.2632 & 300 & -0.010 & 0.0118 & 43 & -0.006 & 0.1153 & 150 \\
\hline Chloride $^{1}$ & 0.007 & -0.001 & 1.0000 & 105 & 0.007 & 0.3593 & 200 & -0.003 & 0.2632 & 90 & -0.003 & $<0.0001$ & 70 \\
\hline Nitrate $^{1}$ & 0.040 & 0.001 & 0.2379 & 33 & 0.022 & 0.5034 & 413 & 0.037 & 0.0636 & 136 & 0.001 & 0.1153 & 36 \\
\hline Sulfate $^{1}$ & 0.036 & 0.000 & 0.5034 & 151 & -0.048 & 0.0044 & 143 & 0.025 & 0.0414 & 60 & -0.019 & 0.0074 & 53 \\
\hline Hydrogen ion ${ }^{2}$ & 2.922 & -0.175 & 0.5034 & 41 & 0.013 & 0.5034 & 46 & 0.664 & 0.8238 & 118 & 1.856 & 0.0004 & 41 \\
\hline Spec. cond. ${ }^{3}$ & 1.030 & -- & -- & -- & 0.890 & 0.0001 & 44 & -0.365 & $<0.0001$ & 54 & -0.275 & 0.5034 & 74 \\
\hline
\end{tabular}

${ }^{1} \mathrm{mg} / \mathrm{L}$, milligrams per liter.

${ }^{2}$ microequivalents per liter,

${ }^{3}$ microSiemens per centimeter.

${ }^{4}$ Overall $f$-psig, $f$-pseudosigma calculated for all results from all participating laboratories.

${ }^{5}$ Median diff., median of differences between each laboratory's individual results and the most probable value (MPV), which is defined as the median of all results from all participating laboratories during 2009.

${ }^{6} f$-psig ratio, ratio of each individual laboratory's $f$-pseudosigma to the overall $f$-pseudosigma, expressed as a percentage. 
Table 6. Comparison of differences between reported concentrations and most probable values for synthetic wet-deposition samples in the 2010 interlaboratorycomparison program.

$[\%$, percent; sign test $p$-value, probability of rejecting the null hypothesis: The true median of the differences between laboratory results and the most probable value is zero, when true; values are shaded where the bias is greater than the method detection limit and is statistically significant ( $\alpha=0.05)$ (Kanji, 1993); Spec. cond., specific conductance; ACAP, Asia Center for Air and Precipitation Reasearch; CAL, Central Analytical Laboratory, Illinois State Water Survey; MACTEC, MACTEC, Inc.; MOEE, Ontario Ministry of Environment and Energy; ECST, Environment Canada Science and Technology Branch; NILU, Norwegian Institute for Air Research; NYSDEC, New York State Department of Environmental Conservation; SA, Shepard Analytical; --, not calculated; $<$, less than]

\begin{tabular}{|c|c|c|c|c|c|c|c|c|c|c|c|c|c|}
\hline \multirow[b]{3}{*}{ Analyte } & \multirow[b]{3}{*}{$\begin{array}{c}\text { Overall } \\
\text { f-psig. }^{4} \\
\text { (units }^{1,2,3} \text { ) }\end{array}$} & \multicolumn{12}{|c|}{ Laboratory } \\
\hline & & \multicolumn{3}{|c|}{ ACAP } & \multicolumn{3}{|c|}{ CAL } & \multicolumn{3}{|c|}{ MACTEC } & \multicolumn{3}{|c|}{ MOEE } \\
\hline & & $\begin{array}{c}\text { Median } \\
\text { diff. }^{5} \\
\text { (units }^{1,2,3} \text { ) }\end{array}$ & $\begin{array}{c}\text { sign } \\
\text { test } \\
p \text {-value }\end{array}$ & $\begin{array}{c}f \text {-psig } \\
\text { ratio }^{6} \\
(\%)\end{array}$ & $\begin{array}{c}\text { Median } \\
\text { diff. }^{5} \\
\text { (units }^{1,2,3} \text { ) }\end{array}$ & $\begin{array}{c}\text { sign } \\
\text { test } \\
p \text {-value }\end{array}$ & $\begin{array}{c}f \text {-psig } \\
\text { ratio }^{6} \\
(\%)\end{array}$ & $\begin{array}{c}\text { Median } \\
\text { diff. }^{5} \\
\text { (units }^{1,2,3} \text { ) }\end{array}$ & $\begin{array}{c}\text { sign } \\
\text { test } \\
p \text {-value }\end{array}$ & $\begin{array}{c}f \text {-psig } \\
\text { ratio }^{6} \\
(\%)\end{array}$ & $\begin{array}{c}\text { Median } \\
\text { diff. }^{5} \\
\text { (units }^{1,2,3} \text { ) }\end{array}$ & $\begin{array}{c}\text { sign } \\
\text { test } \\
p \text {-value }\end{array}$ & $\begin{array}{c}\text { f-psig } \\
\text { ratio }^{6} \\
(\%)\end{array}$ \\
\hline Calcium $^{1}$ & 0.014 & 0.016 & $<0.0001$ & 68 & 0.001 & 0.1671 & 14 & -0.006 & 0.1153 & 79 & -0.002 & 1.0000 & 39 \\
\hline Magnesium $^{1}$ & 0.003 & -0.003 & 0.0414 & 56 & 0.000 & 0.0215 & 19 & 0.000 & 1.0000 & 38 & 0.001 & 0.1153 & 194 \\
\hline Sodium ${ }^{1}$ & 0.007 & 0.024 & $<0.0001$ & 55 & 0.001 & 0.0309 & 40 & 0.001 & 0.2668 & 20 & 0.001 & 0.2101 & 40 \\
\hline Potassium $^{1}$ & 0.004 & 0.000 & 1.0000 & 63 & 0.000 & 0.0034 & 17 & 0.001 & 0.0018 & 75 & -0.004 & 0.2632 & 125 \\
\hline Ammonium $^{1}$ & 0.010 & -0.002 & 0.0005 & 54 & 0.004 & 0.0034 & 43 & -0.001 & 0.1435 & 13 & 0.002 & 0.3018 & 64 \\
\hline Chloride $^{1}$ & 0.010 & -0.006 & $<0.0001$ & 93 & 0.002 & 0.0963 & 21 & -0.001 & 1.0000 & 13 & 0.010 & 0.8238 & 293 \\
\hline Nitrate $^{1}$ & 0.033 & -0.025 & 0.0118 & 82 & -0.008 & 0.1153 & 53 & 0.012 & 0.6476 & 61 & -0.006 & 1.0000 & 95 \\
\hline Sulfate ${ }^{1}$ & 0.031 & -0.032 & $<0.0001$ & 48 & -0.004 & 0.8238 & 34 & -0.002 & 0.6291 & 59 & 0.020 & 0.0192 & 139 \\
\hline Hydrogen ion ${ }^{2}$ & 2.644 & -2.684 & 0.0026 & 73 & 0.266 & 0.2632 & 23 & -3.298 & $<0.0001$ & 103 & 1.014 & 0.0414 & 51 \\
\hline Spec. cond. ${ }^{3}$ & 1.186 & -0.250 & 0.4807 & 47 & 0.450 & 0.0001 & 25 & 0.810 & $<0.0001$ & 49 & -1.600 & $<0.0001$ & 41 \\
\hline Calcium $^{1}$ & 0.014 & 0.004 & 0.1153 & 62 & -0.004 & 0.1153 & 133 & -0.019 & 0.0227 & 132 & 0.010 & 0.1250 & 91 \\
\hline Magnesium $^{1}$ & 0.003 & 0.000 & 0.3877 & 25 & -0.003 & 0.2632 & 74 & -0.007 & 0.0639 & 300 & 0.001 & 1.0000 & 100 \\
\hline Sodium $^{1}$ & 0.007 & 0.000 & 0.4807 & 50 & -0.005 & 0.2632 & 642 & -0.008 & 0.0227 & 60 & -0.002 & 0.3750 & 50 \\
\hline Potassium $^{1}$ & 0.004 & -0.005 & 0.0414 & 113 & 0.002 & 0.8238 & 350 & -0.007 & 0.0169 & 100 & -0.002 & 0.0156 & 33 \\
\hline Ammonium $^{1}$ & 0.010 & 0.008 & 0.0309 & 91 & -0.034 & 0.1153 & 393 & -0.006 & 0.0931 & 114 & -0.006 & 0.0625 & 43 \\
\hline Chloride $^{1}$ & 0.010 & -0.001 & 1.0000 & 57 & -0.004 & 0.1671 & 427 & -0.008 & 0.0066 & 79 & -0.004 & 0.1250 & 170 \\
\hline Nitrate $^{1}$ & 0.033 & 0.000 & 0.5034 & 56 & -0.023 & 0.0414 & 274 & -0.017 & 0.5413 & 114 & 0.010 & 1.0000 & 80 \\
\hline Sulfate $^{1}$ & 0.031 & -0.020 & 0.0414 & 28 & -0.018 & 0.8238 & 229 & 0.002 & 0.5413 & 131 & -0.015 & 0.2188 & 73 \\
\hline Hydrogen ion ${ }^{2}$ & 2.644 & -2.038 & 0.1153 & 99 & 0.337 & 0.2632 & 37 & 0.194 & 0.1516 & 37 & 0.995 & 0.0156 & 54 \\
\hline Spec. cond. ${ }^{3}$ & 1.186 & -- & -- & -- & 0.860 & 0.0118 & 34 & -0.930 & $<0.0001$ & 50 & 0.050 & 1.0000 & 34 \\
\hline
\end{tabular}

${ }^{1} \mathrm{mg} / \mathrm{L}$, milligrams per liter.

${ }^{2}$ microequivalents per liter.

${ }^{3}$ microSiemens per centimeter.

${ }^{4}$ Overall $f$-psig, $f$-pseudosigma calculated for all results from all participating laboratories.

${ }^{5}$ Median diff., median of differences between each laboratory's individual results and the most probable value (MPV), which is defined as the median of all results from all participating laboratories during 2010.

${ }^{6} f$-psig ratio, ratio of each individual laboratory's $f$-pseudosigma to the overall $f$-pseudosigma, expressed as a percentage. 
Table 7. Number of analyte determinations greater than the method detection limits in deionized-water samples for each participating laboratory and method detection limits for participating laboratories during 2009-10.

[ACAP, Asia Center for Air Pollution Research ; CAL, Central Analytical Laboratory, Illinois State Water Survey; MACTEC, MACTEC, Inc.; MOEE, Ontario Ministry of Environment and Energy; ECST, Environment Canada Science and Technology Branch; NILU, Norwegian Institute for Air Research; NYSDEC, New York State Department of Environmental Conservation; SA, Shepard Analytical; mg/L, milligrams per liter]

\begin{tabular}{|c|c|c|c|c|c|c|c|c|}
\hline Analyte & ACAP & CAL & MACTEC & MOEE & ECST & NILU & NYSDEC & SA \\
\hline & \multicolumn{8}{|c|}{2009} \\
\hline Calcium & 1 & 0 & 0 & 0 & 0 & 0 & 0 & 0 \\
\hline Magnesium & 0 & 0 & 0 & 0 & 0 & 0 & 0 & 0 \\
\hline Sodium & 0 & 0 & 0 & 0 & 0 & 0 & 0 & 0 \\
\hline Potassium & 3 & 0 & 0 & 0 & 0 & 0 & 0 & 0 \\
\hline Ammonium & 2 & 0 & 0 & 2 & 0 & 1 & 0 & 0 \\
\hline Chloride & 1 & 0 & 0 & 0 & 0 & 1 & 0 & 0 \\
\hline Nitrate & 0 & 0 & 0 & 0 & 0 & 1 & 0 & 0 \\
\hline \multirow[t]{2}{*}{ Sulfate } & 0 & 0 & 0 & 0 & 0 & 1 & 0 & 0 \\
\hline & \multicolumn{8}{|c|}{2010} \\
\hline Calcium & 4 & 0 & 0 & 0 & 0 & 1 & 1 & 0 \\
\hline Magnesium & 2 & 0 & 0 & 0 & 0 & 1 & 1 & 0 \\
\hline Sodium & 4 & 0 & 0 & 0 & 0 & 1 & 1 & 0 \\
\hline Potassium & 1 & 0 & 0 & 0 & 0 & 1 & 1 & 0 \\
\hline Ammonium & 0 & 0 & 0 & 2 & 0 & 1 & 1 & 0 \\
\hline Chloride & 0 & 0 & 0 & 0 & 0 & 2 & 1 & 0 \\
\hline Nitrate & 0 & 0 & 0 & 0 & 0 & 2 & 1 & 0 \\
\hline \multirow[t]{3}{*}{ Sulfate } & 0 & 0 & 0 & 0 & 0 & 2 & 1 & 0 \\
\hline & \multicolumn{8}{|c|}{ Method Detection Limits (mg/L) } \\
\hline & \multicolumn{8}{|c|}{$2009 / 2010$} \\
\hline Calcium & 0.002 & 0.006 & 0.003 & 0.100 & 0.020 & 0.010 & 0.010 & 0.020 \\
\hline Magnesium & 0.006 & 0.001 & 0.003 & 0.025 & 0.010 & 0.010 & 0.010 & 0.002 \\
\hline Sodium & 0.009 & 0.001 & 0.005 & 0.025 & 0.020 & 0.010 & 0.010 & 0.001 \\
\hline Potassium & 0.003 & 0.001 & 0.005 & 0.025 & 0.020 & 0.010 & 0.010 & 0.001 \\
\hline Ammonium & 0.014 & $0.003 / .010$ & 0.020 & 0.010 & 0.006 & 0.010 & 0.010 & 0.005 \\
\hline Chloride & 0.004 & 0.003 & 0.020 & 0.050 & 0.020 & 0.010 & 0.010 & 0.002 \\
\hline Nitrate & 0.002 & 0.005 & 0.035 & 0.040 & 0.030 & 0.010 & 0.010 & 0.010 \\
\hline Sulfate & 0.001 & 0.004 & 0.020 & 0.250 & 0.020 & 0.010 & 0.010 & 0.010 \\
\hline
\end{tabular}


$A$

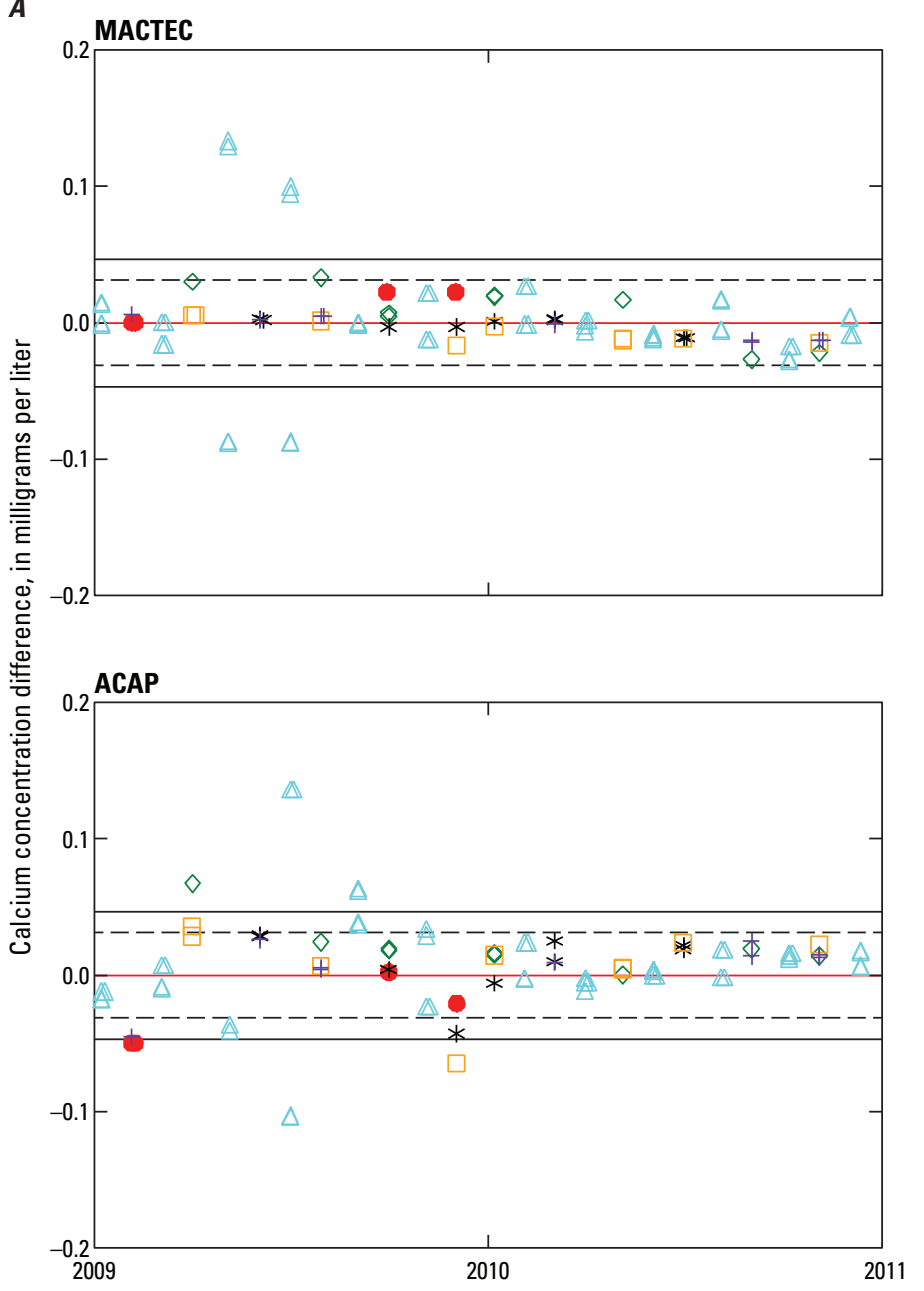

ECST

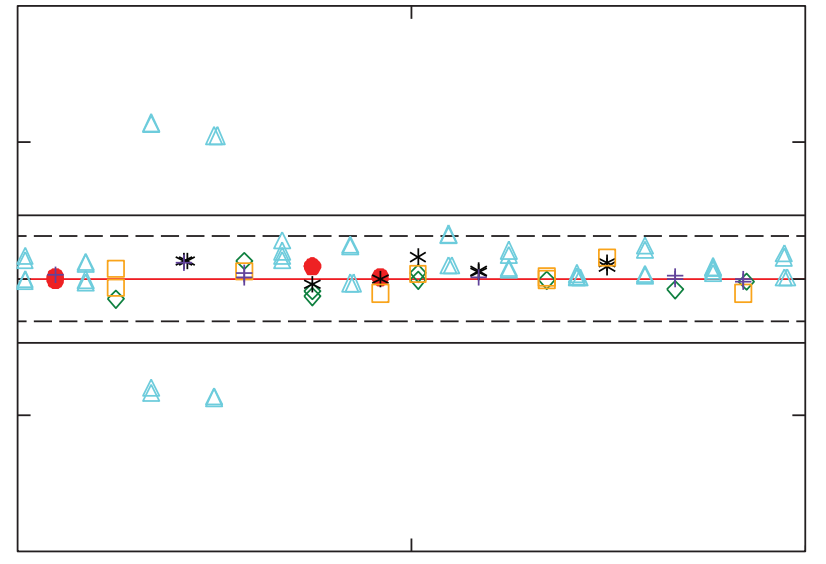

CAL

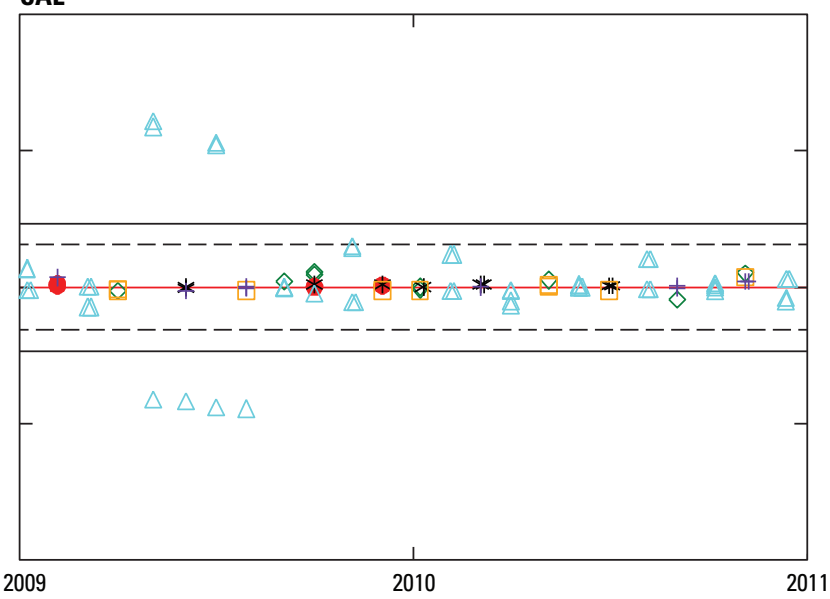

\section{EXPLANATION}

Control limit line -+3 and $-3 f$-pseudosigmas from zero difference

Warning limit line -+2 and $-2 f$-pseudosigmas from zero difference

Zero line

Solution-2009-2010 U.S. Geological Survey interlaboratory-comparison

program, table 4

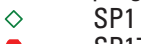

SP1

SP21

SP3

SP97

Natural wet deposition-CALNAT

Laboratories:

MACTEC-MACTEC, Inc., Gainesville, Florida

ECST-Environment Canada Science \& Technology Branch (formerly MSC), Downsview, Ontario, Canada

ACAP_Asia Center for Air and Precipitation Research (formerly ADORC), Niigata-shi, Japan

CAL-Central Analytical Laboratory, Illinois State Water Survey, Champaign, Illinois

Figure 3. A, Difference between the measured calcium concentration values and the median calcium concentration value calculated by solution for all participating laboratories in the interlaboratory-comparison program during 2009-10 for MACTEC, Inc., Environment Canada Science and Technology Branch (ECST), Asia Center for Air and Precipitation Research (ACAP), and Central Analytical Laboratory (CAL) laboratories. 
B

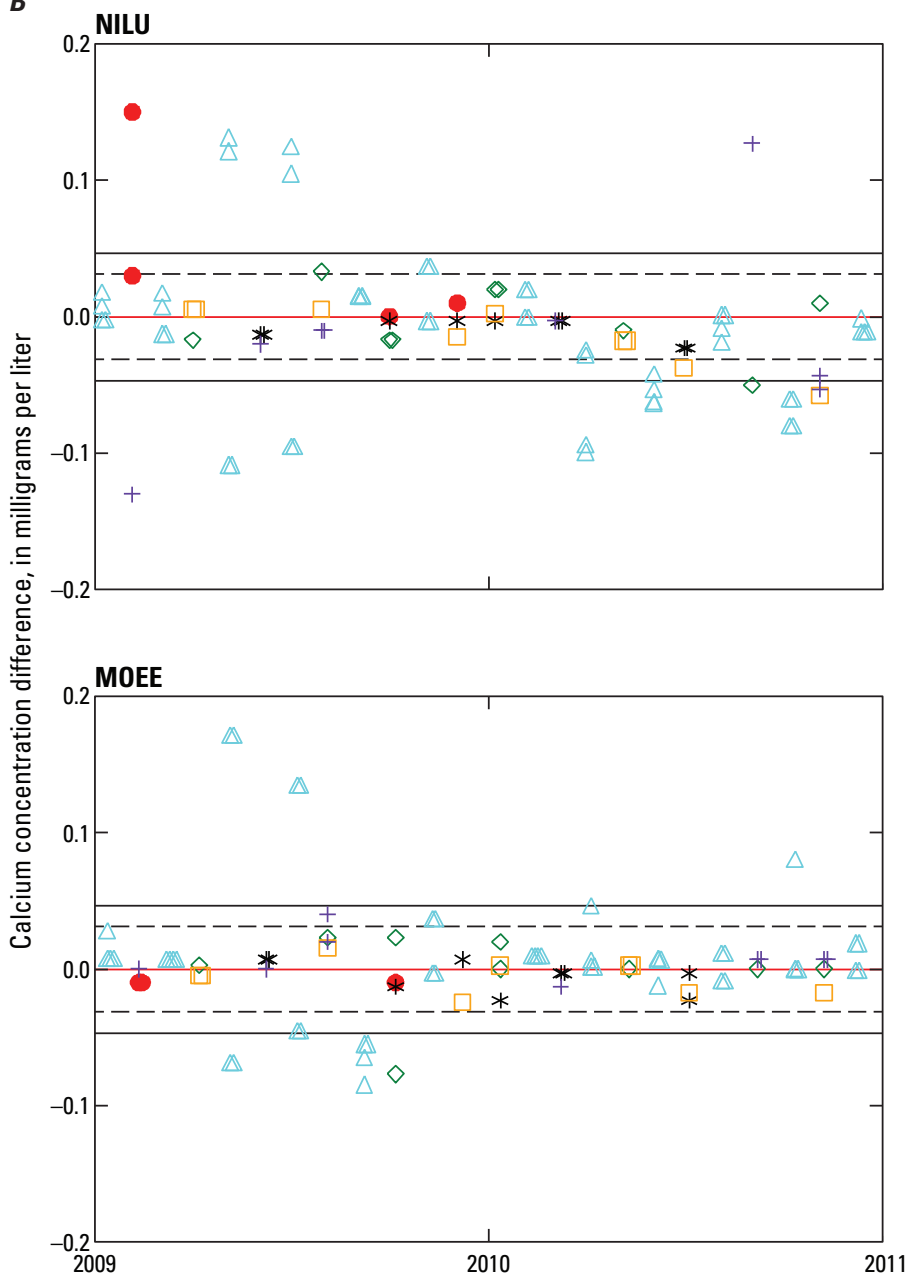

SA

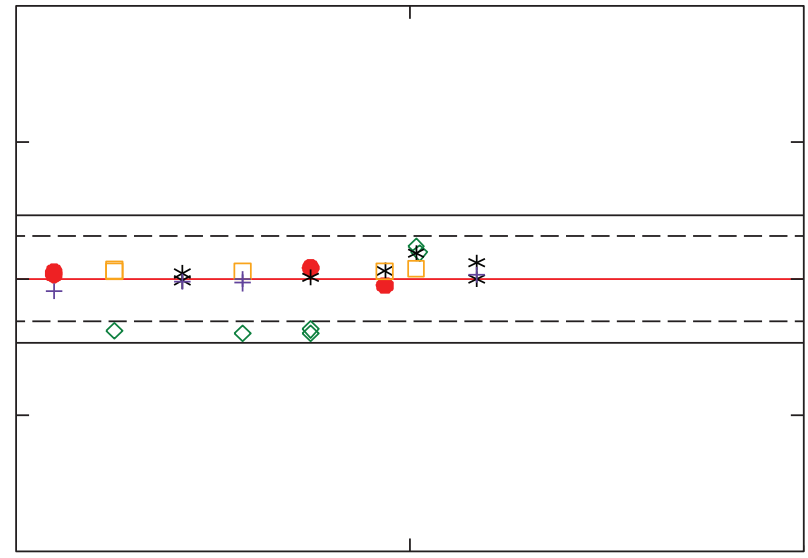

NYSDEC

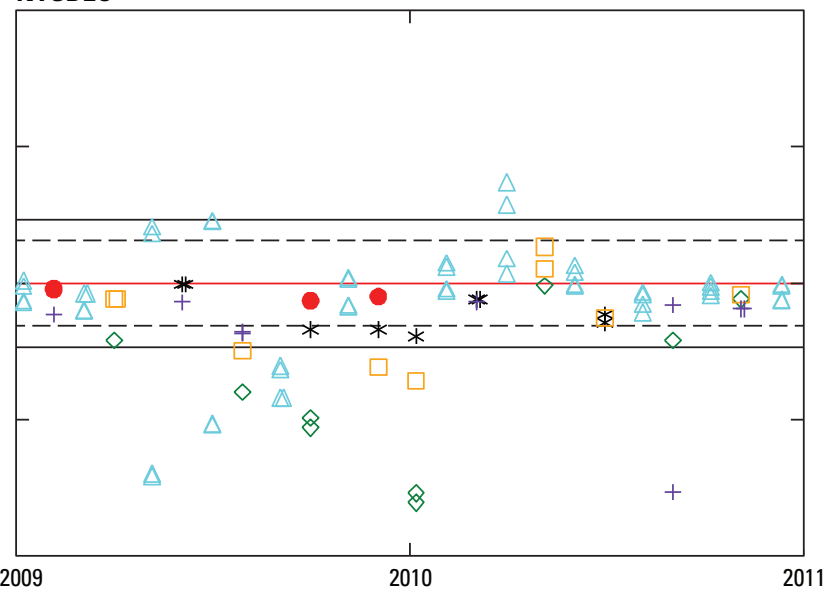

\section{EXPLANATION}

Control limit line -+3 and $-3 f$-pseudosigmas from zero difference

Warning limit line -+2 and $-2 f$-pseudosigmas from zero difference

Zero line

Solution-2009-2010 U.S. Geological Survey interlaboratory-comparison

program, table 4

$\diamond \quad \mathrm{SP} 1$

$\mathrm{SP} 17$

SP21

$\mathrm{SP} 3$

SP97

Natural wet deposition-CALNAT

Laboratories:

NILU—Norwegian Institute for Air Research, Kjeller, Norway

SA-Shepard Analytical, Simi Valley, California

MOEE - Ontario Ministry of Environment and Energy, Dorset Research Facility, Dorset, Ontario, Canada

NYSDEC - New York State Department of Environmental Conservation, Albany, New York

Figure 3. $B$, Difference between the measured calcium concentration values and the median calcium concentration value calculated by solution for all participating laboratories in the interlaboratory-comparison program during 2009-10 for Norwegian Institute for Air Research (NILU), Shephard Analytical (SA), Ontario Ministry of Environment and Energy (MOEE), and New York State Department of Environmental Conservation (NYSDEC) laboratories. 
$\boldsymbol{A}$

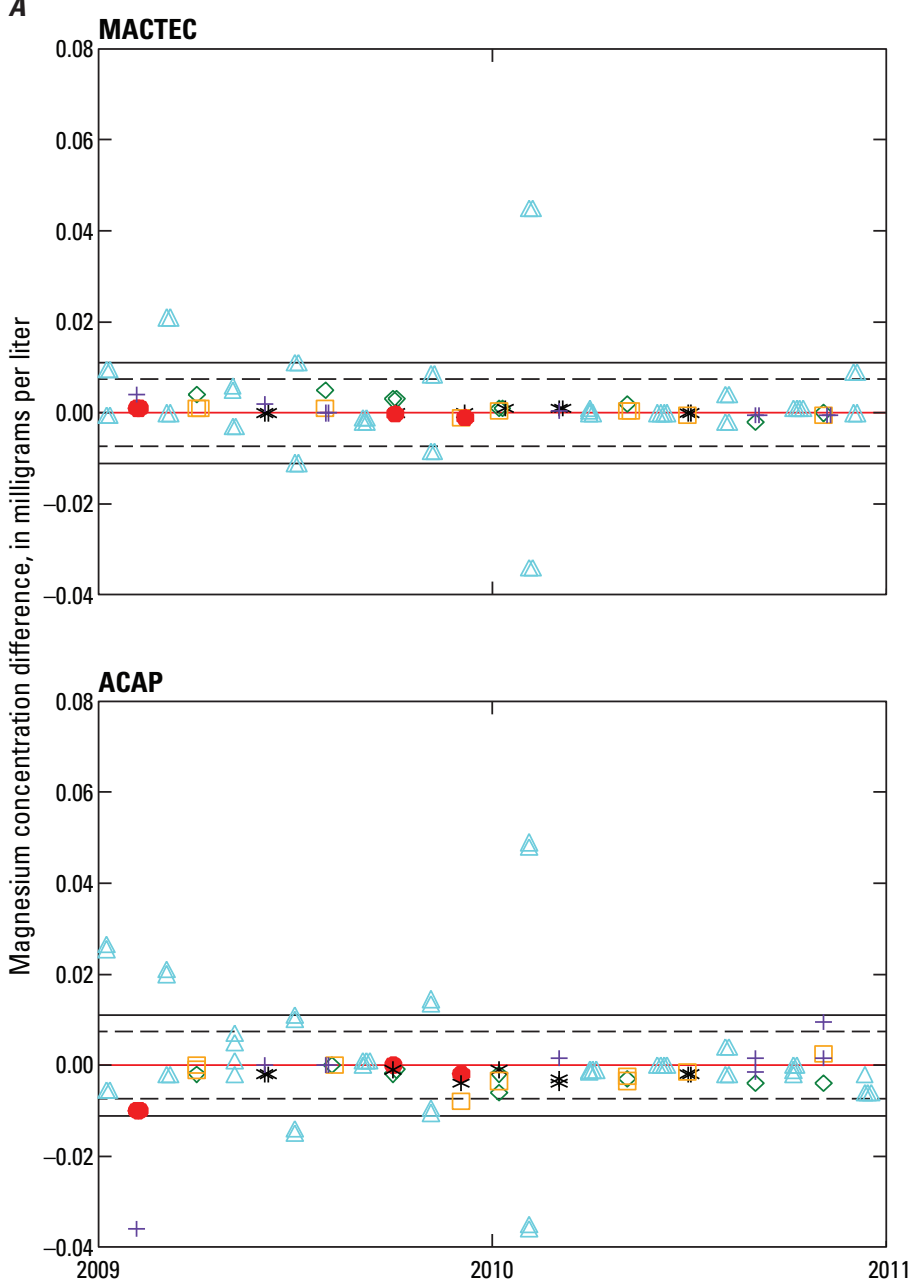

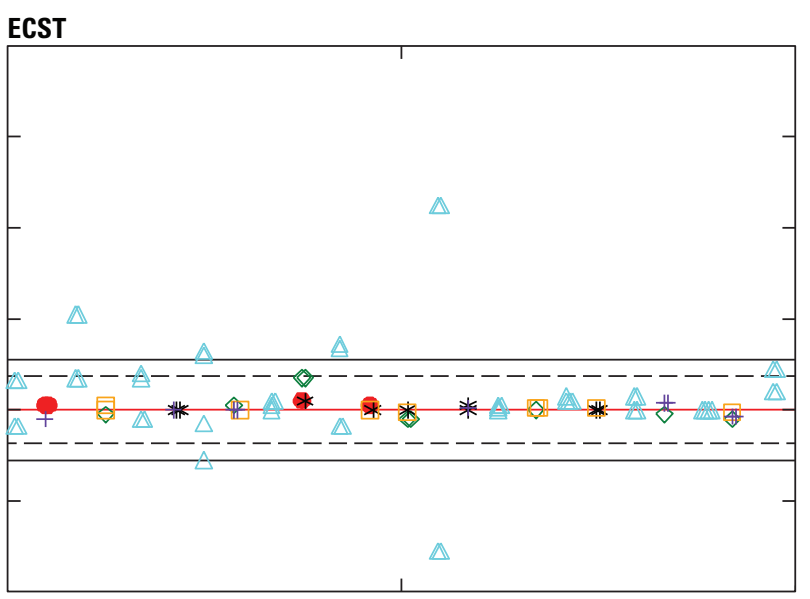

CAL

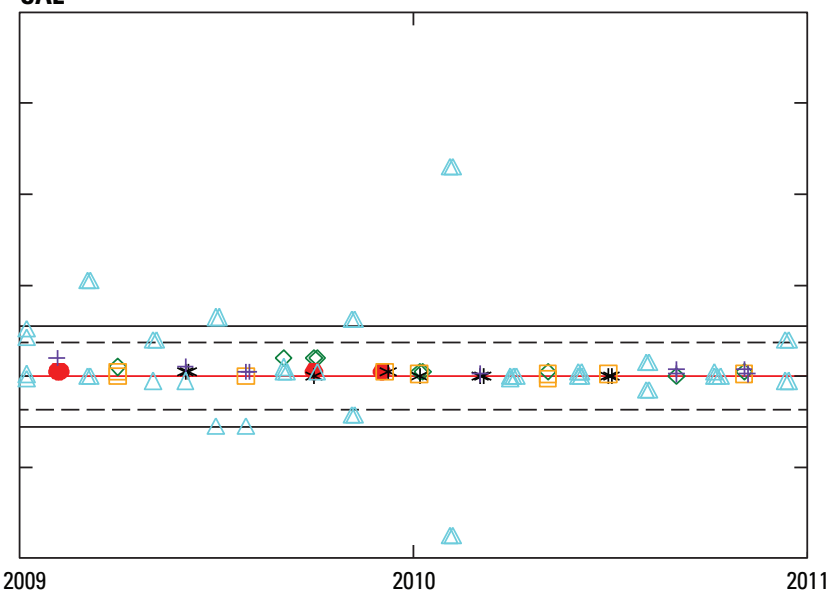

\section{EXPLANATION}

Control limit line -+3 and $-3 f$-pseudosigmas from zero difference

Warning limit line -+2 and $-2 f$-pseudosigmas from zero difference

Zero line

Solution-2009-2010 U.S. Geological Survey interlaboratory-comparison

program, table 4

$\diamond \quad \mathrm{SP} 1$

SP17

SP21

SP3

SP97

Natural wet deposition-CALNAT

Laboratories:

MACTEC_-MACTEC, Inc., Gainesville, Florida

ECST—Environment Canada Science \& Technology Branch (formerly MSC), Downsview, Ontario, Canada

ACAP_ Asia Center for Air and Precipitation Research (formerly ADORC), Niigata-shi, Japan

CAL-Central Analytical Laboratory, Illinois State Water Survey, Champaign, Illinois

Figure 4. $A$, Difference between the measured magnesium concentration values and the median magnesium concentration value calculated by solution for all participating laboratories in the interlaboratory-comparison program during 2009-10 for MACTEC, Inc., Environment Canada Science and Technology Branch (ECST), Asia Center for Air and Precipitation Research (ACAP), and Central Analytical Laboratory (CAL) laboratories. 
$\boldsymbol{B}$

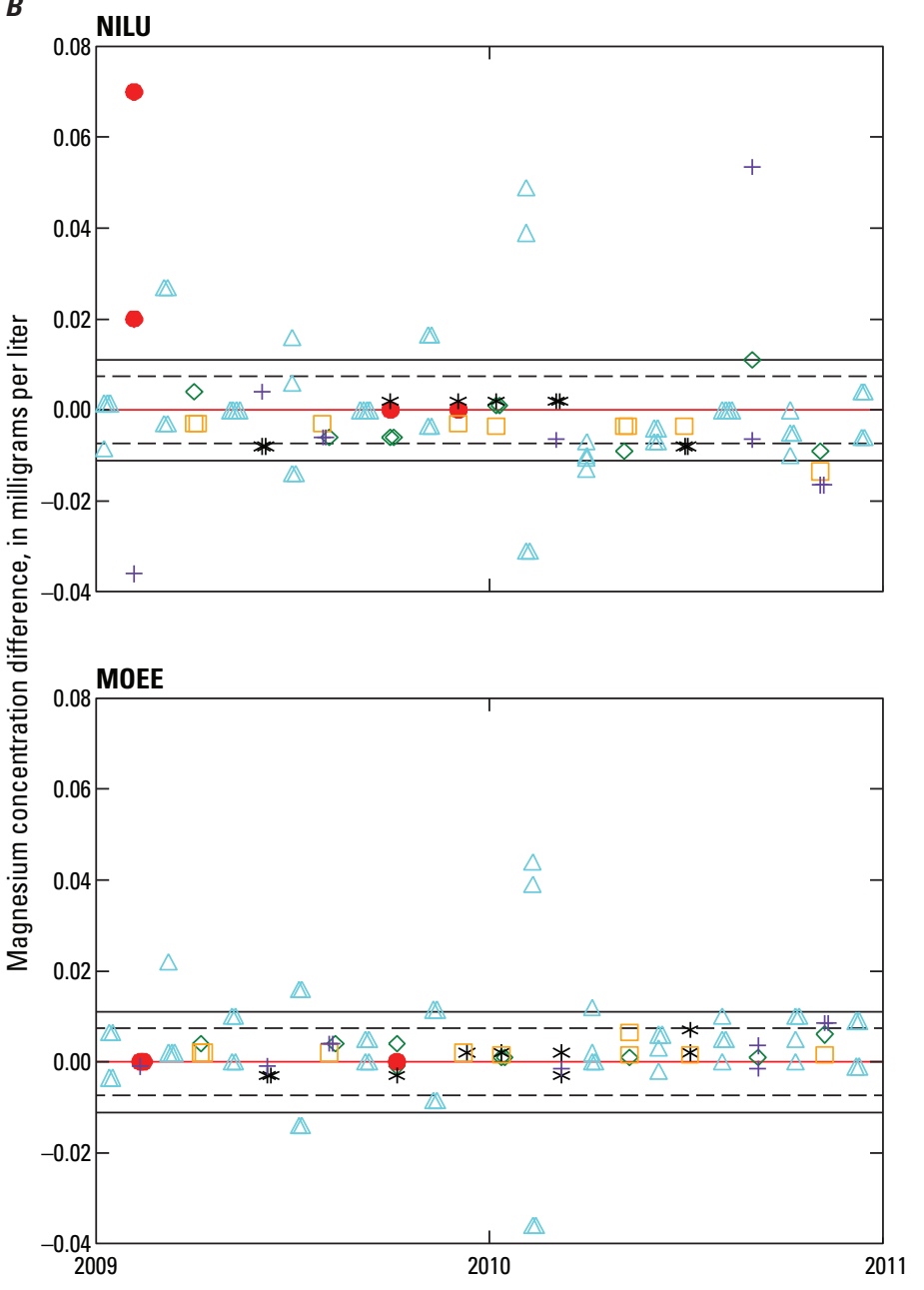

SA

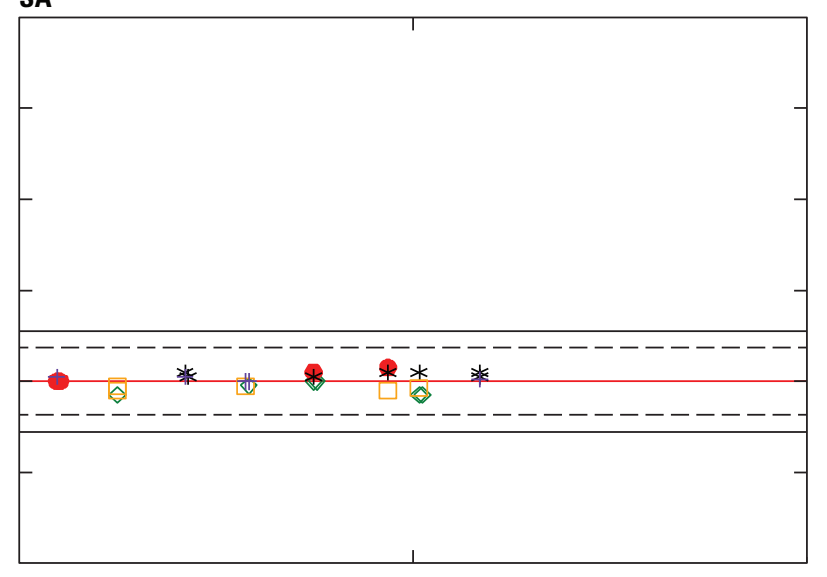

NYSDEC

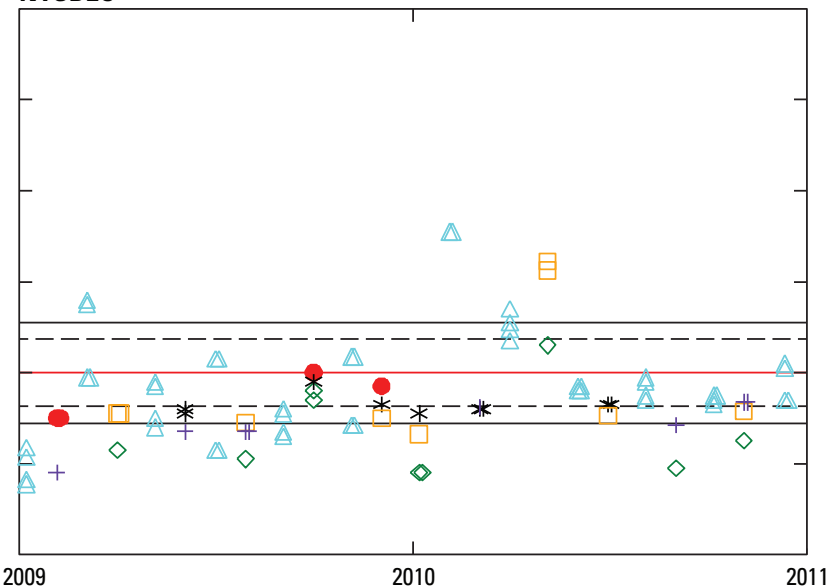

\section{EXPLANATION}

Control limit line -+3 and $-3 f$-pseudosigmas from zero difference

Warning limit line -+2 and $-2 f$-pseudosigmas from zero difference

Zero line

Solution-2009-2010 U.S. Geological Survey interlaboratory-comparison

program, table 4

$\diamond \quad$ SP1

- SP17

SP21

SP3

SP97

Natural wet deposition-CALNAT

Laboratories:

NILU—Norwegian Institute for Air Research, Kjeller, Norway

SA-Shepard Analytical, Simi Valley, California

MOEE-Ontario Ministry of Environment and Energy, Dorset Research Facility, Dorset, Ontario, Canada

NYSDEC — New York State Department of Environmental Conservation, Albany, New York

Figure 4. $\quad B$, Difference between the measured magnesium concentration values and the median magnesium concentration value calculated by solution for all participating laboratories in the interlaboratory-comparison program during 2009-10 for Norwegian Institute for Air Research (NILU), Shephard Analytical (SA), Ontario Ministry of Environment and Energy (MOEE), and New York State Department of Environmental Conservation (NYSDEC) laboratories. 
$\boldsymbol{A}$

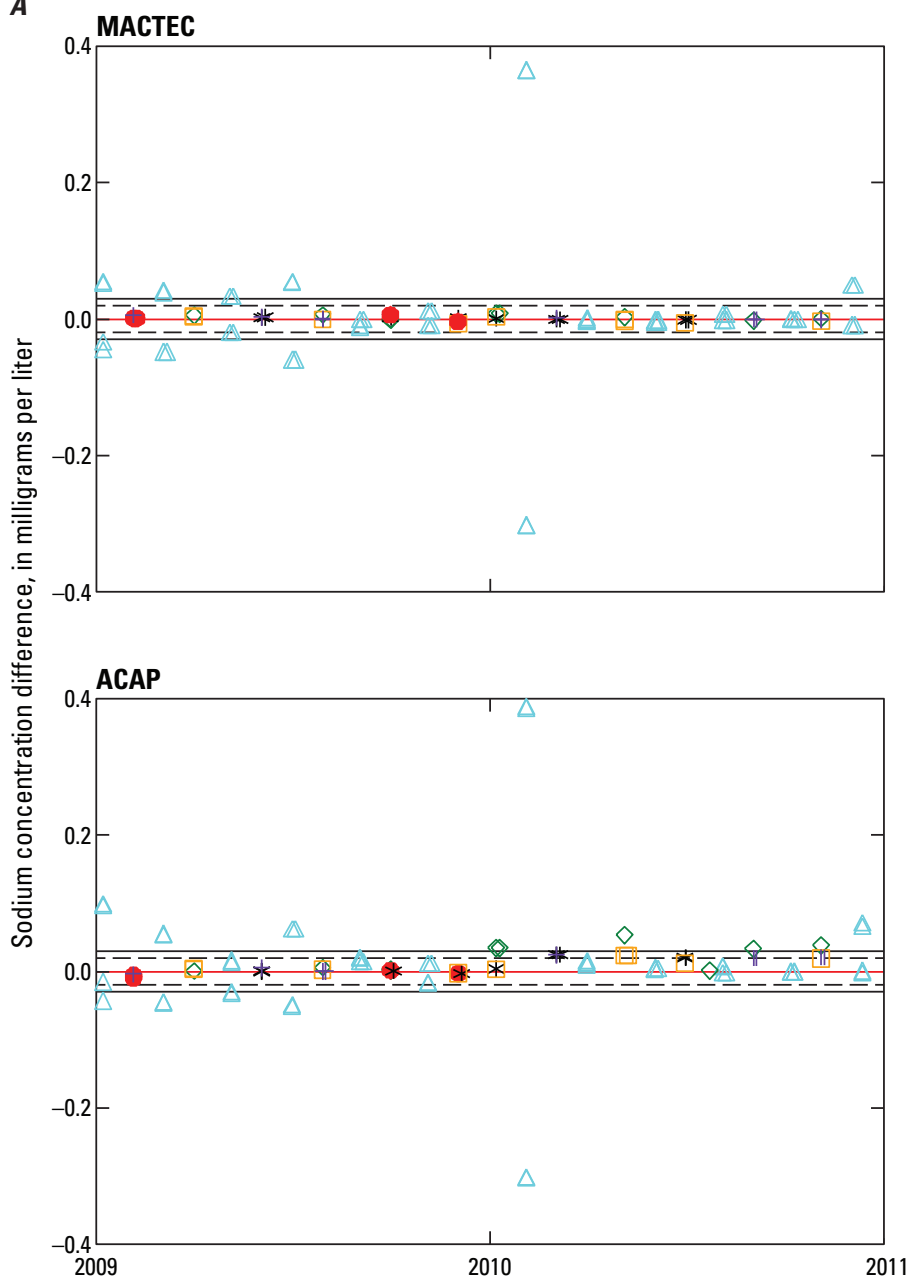

ECST

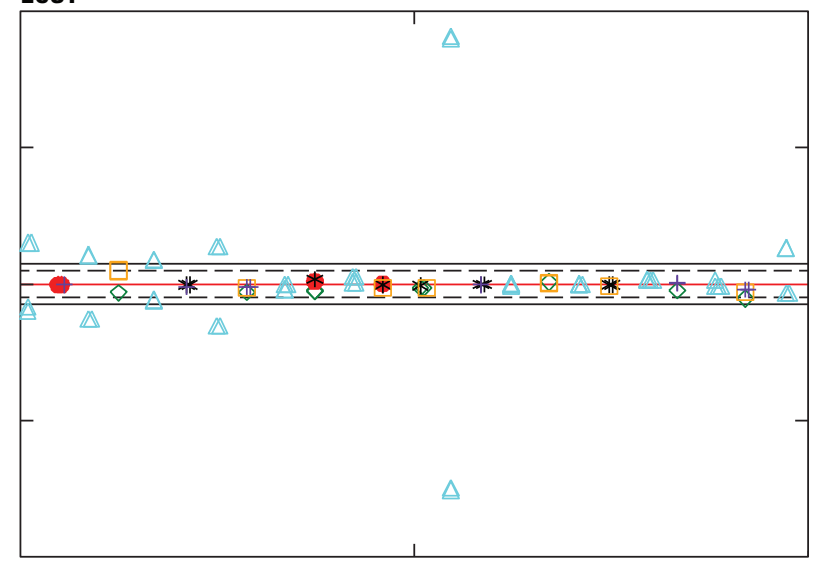

CAL

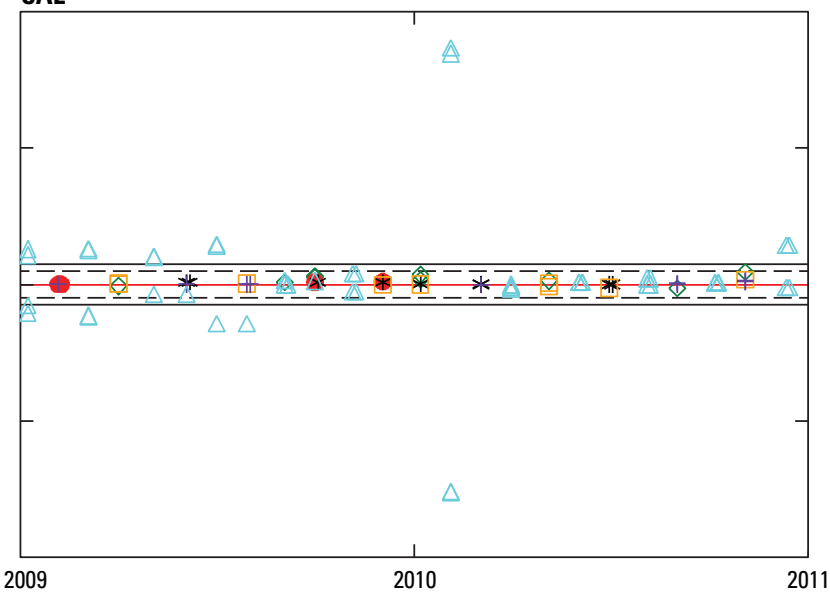

Year

\section{EXPLANATION}

Control limit line -+3 and $-3 f$-pseudosigmas from zero difference

Warning limit line -+2 and $-2 f$-pseudosigmas from zero difference

Zero line

Solution-2009-2010 U.S. Geological Survey interlaboratory-comparison

program, table 4

$\diamond \quad \mathrm{SP} 1$

SP17

SP21

SP3

SP97

Natural wet deposition-CALNAT

Laboratories:

MACTEC-MACTEC, Inc., Gainesville, Florida

ECST—Environment Canada Science \& Technology Branch (formerly MSC), Downsview, Ontario, Canada

ACAP_Asia Center for Air and Precipitation Research (formerly ADORC), Niigata-shi, Japan

CAL-Central Analytical Laboratory, Illinois State Water Survey, Champaign, Illinois

Figure 5. A, Difference between the measured sodium concentration values and the median sodium concentration value calculated by solution for all participating laboratories in the interlaboratory-comparison program during 2009-10 for MACTEC, Inc., Environment Canada Science and Technology Branch (ECST), Asia Center for Air and Precipitation Research (ACAP), and Central Analytical Laboratory (CAL) laboratories. 
$B$
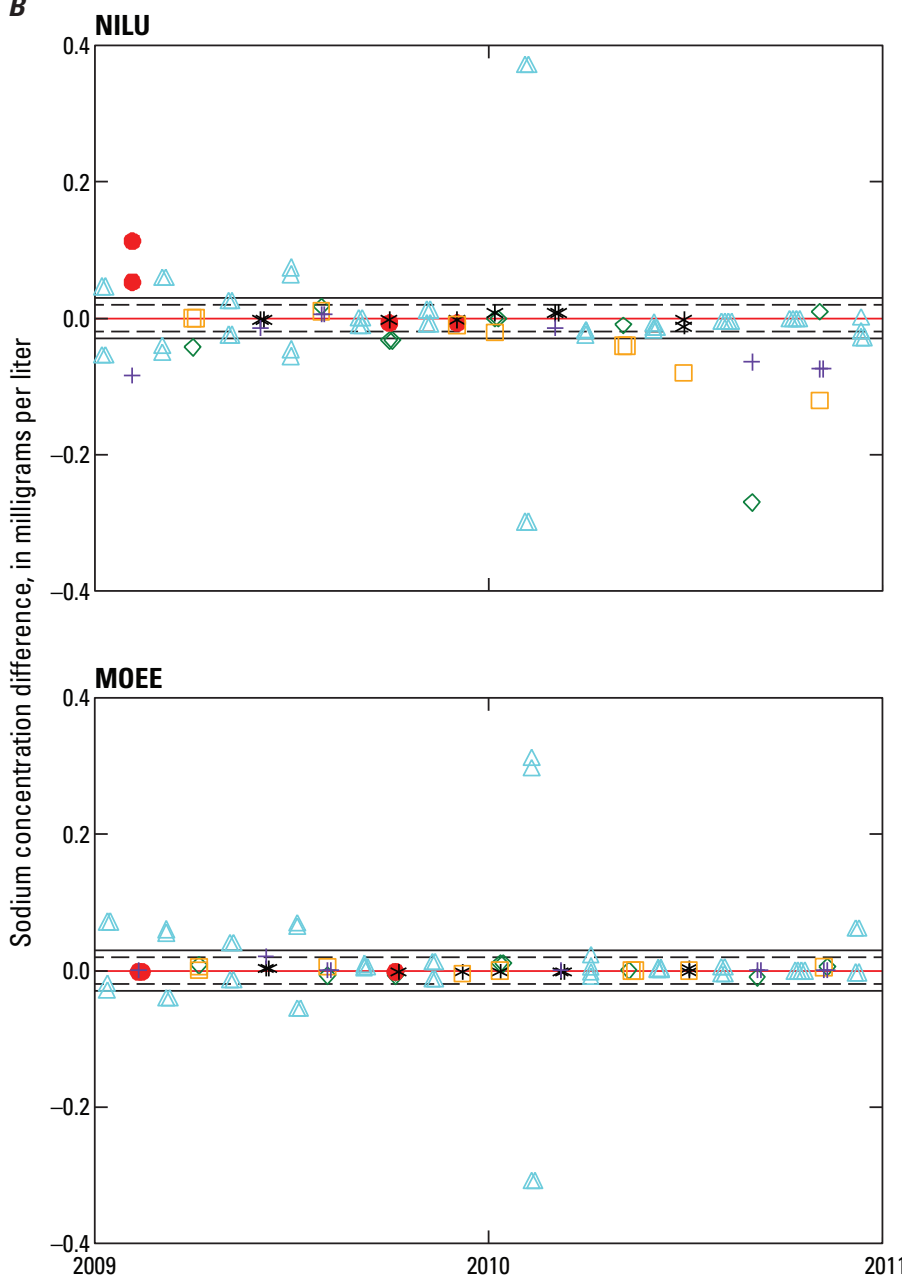

SA

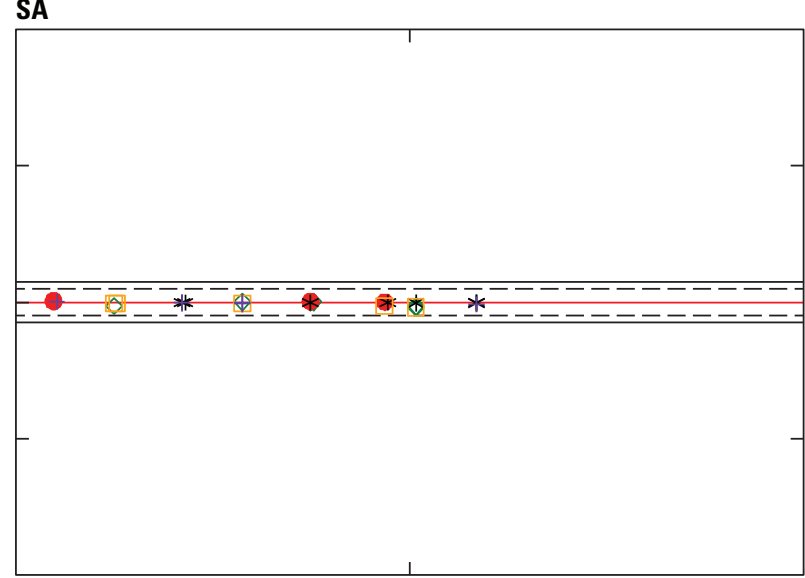

NYSDEC

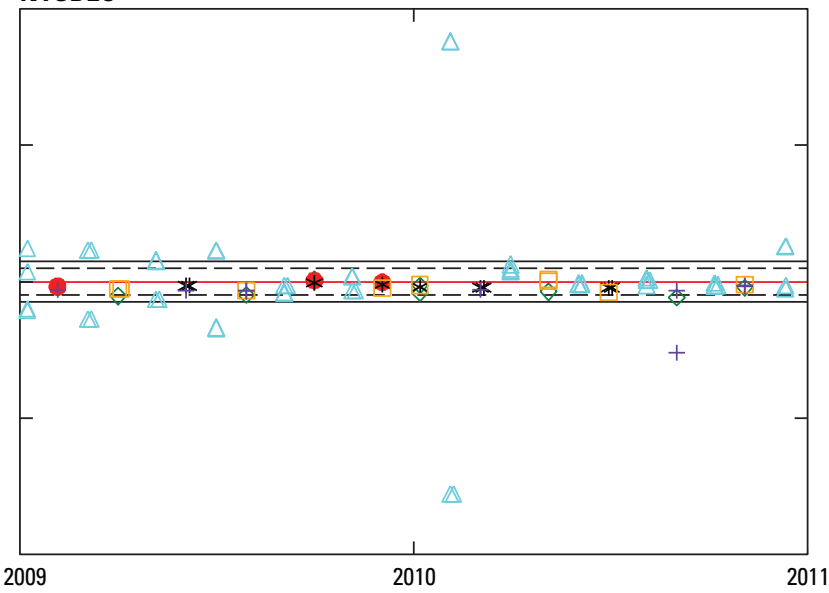

Year

\section{EXPLANATION}

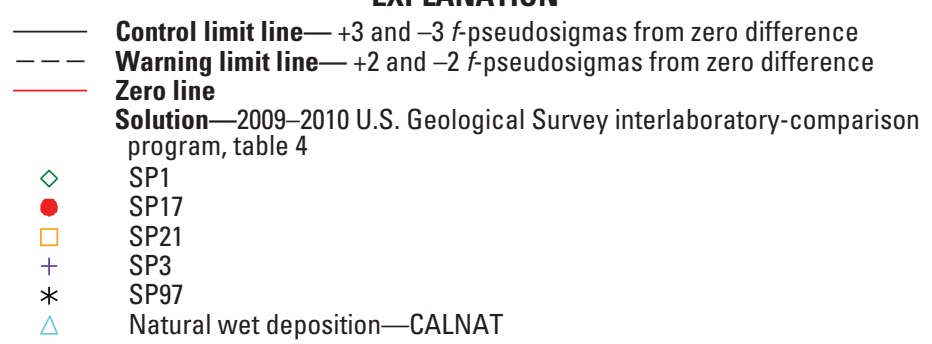

Laboratories:

NILU—Norwegian Institute for Air Research, Kjeller, Norway

SA-Shepard Analytical, Simi Valley, California

MOEE-Ontario Ministry of Environment and Energy, Dorset Research Facility, Dorset, Ontario, Canada

NYSDEC - New York State Department of Environmental Conservation, Albany, New York

Figure 5. $B$, Difference between the measured sodium concentration values and the median sodium concentration value calculated by solution for all participating laboratories in the interlaboratory-comparison program during 2009-10 for Norwegian Institute for Air Research (NILU), Shephard Analytical (SA), Ontario Ministry of Environment and Energy (MOEE), and New York State Department of Environmental Conservation (NYSDEC) laboratories. 
$A$

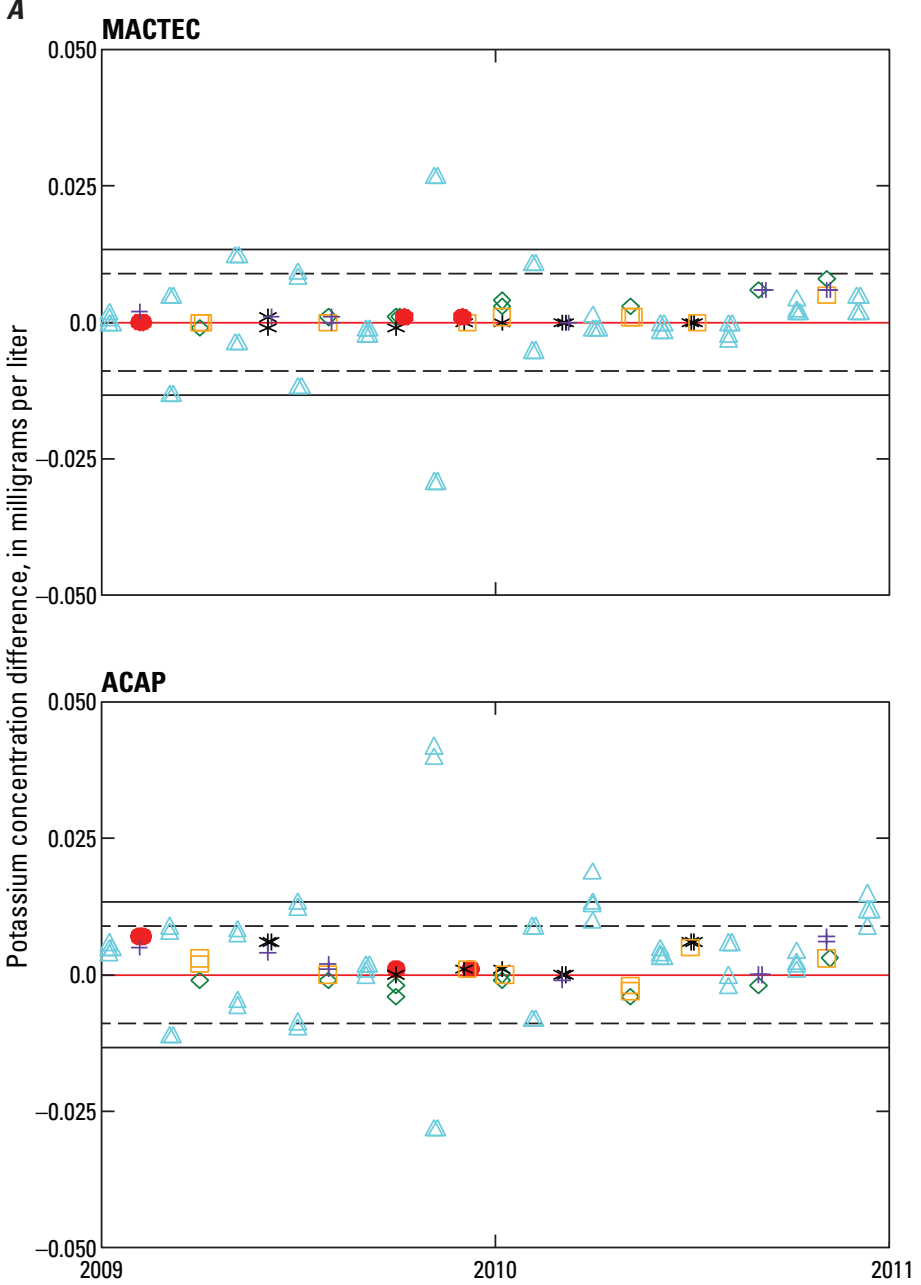

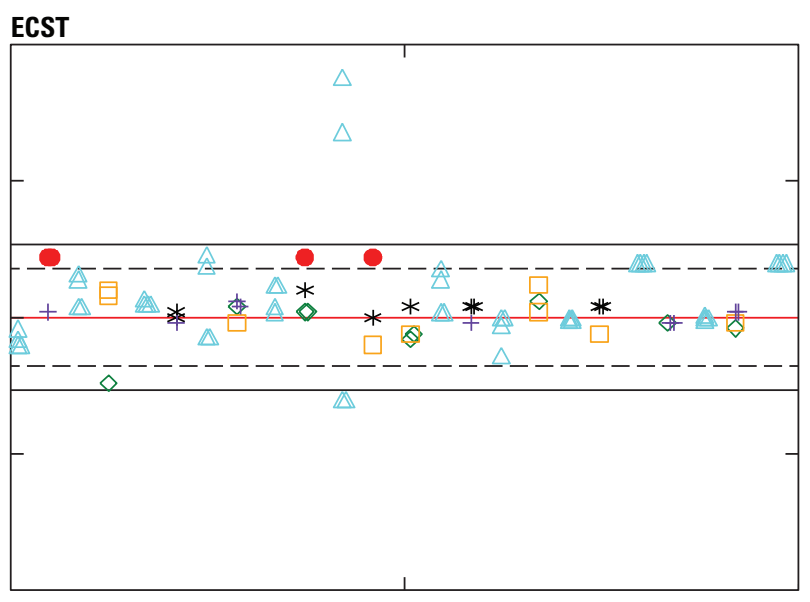

CAL

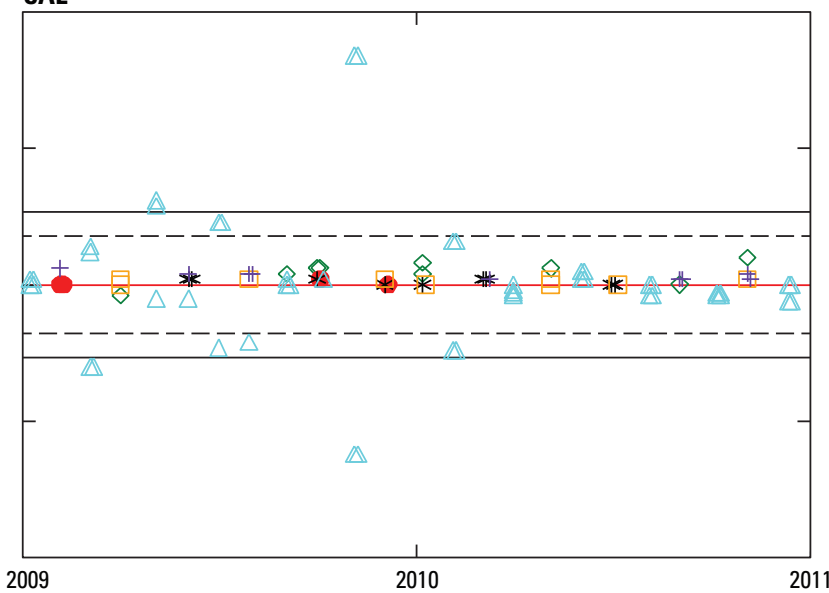

Year

\section{EXPLANATION}

Control limit line -+3 and $-3 f$-pseudosigmas from zero difference

Warning limit line- +2 and $-2 f$-pseudosigmas from zero difference

Zero line

Solution-2009-2010 U.S. Geological Survey interlaboratory-comparison

program, table 4

$\diamond \quad$ SP1

SP17

SP21

SP3

SP97

Natural wet deposition-CALNAT

Laboratories:

MACTEC-MACTEC, Inc., Gainesville, Florida

ECST-Environment Canada Science \& Technology Branch (formerly MSC), Downsview, Ontario, Canada

ACAP_Asia Center for Air and Precipitation Research (formerly ADORC), Niigata-shi, Japan

CAL-Central Analytical Laboratory, Illinois State Water Survey, Champaign, Illinois

Figure 6. $A$, Difference between the measured potassium concentration values and the median potassium concentration value calculated by solution for all participating laboratories in the interlaboratory-comparison program during 2009-10 for MACTEC, Inc., Environment Canada Science and Technology Branch (ECST), Asia Center for Air and Precipitation Research (ACAP), and Central Analytical Laboratory (CAL) laboratories. 
$B$

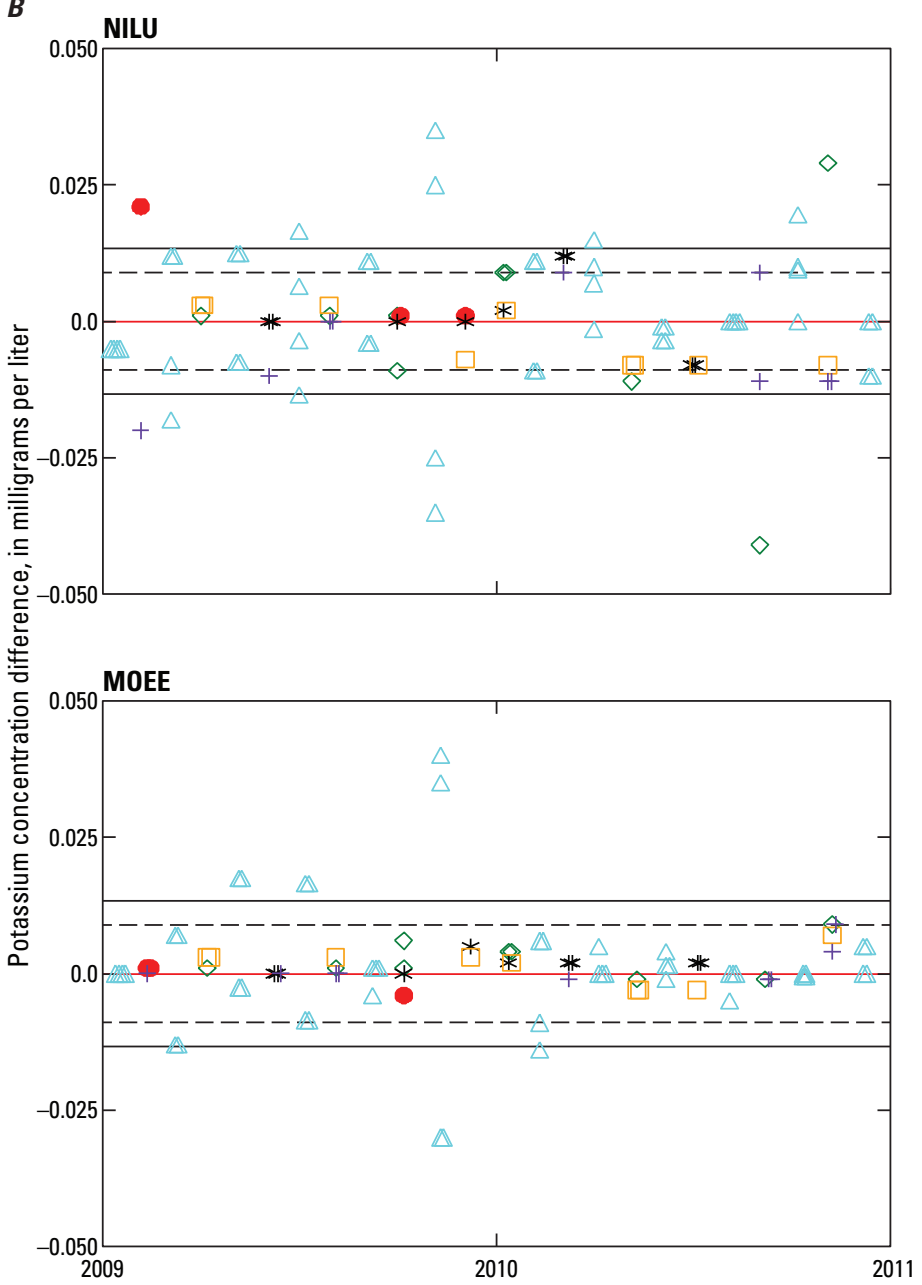

SA

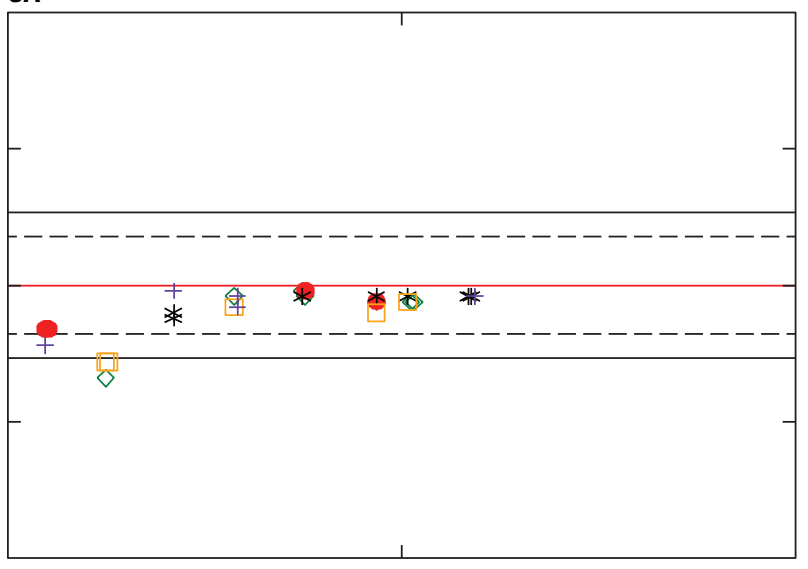

NYSDEC

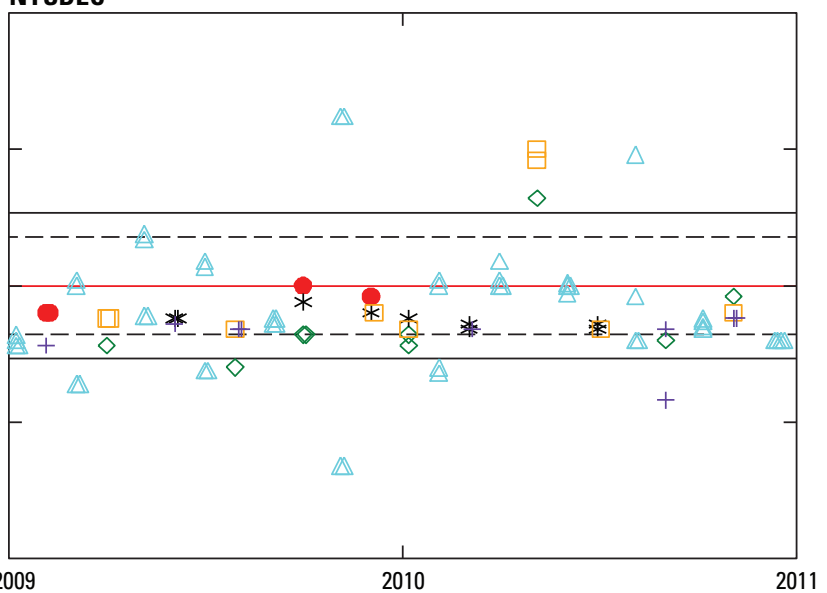

Year

\section{EXPLANATION}

Control limit line -+3 and $-3 f$-pseudosigmas from zero difference

Warning limit line- +2 and $-2 f$-pseudosigmas from zero difference

Zero line

Solution-2009-2010 U.S. Geological Survey interlaboratory-comparison

program, table 4

$\diamond \quad$ SP1

SP17

SP21

SP3

SP97

Natural wet deposition-CALNAT

Laboratories:

NILU—Norwegian Institute for Air Research, Kjeller, Norway

SA-Shepard Analytical, Simi Valley, California

MOEE- Ontario Ministry of Environment and Energy, Dorset Research Facility, Dorset, Ontario, Canada

NYSDEC - New York State Department of Environmental Conservation, Albany, New York

Figure 6. $B$, Difference between the measured potassium concentration values and the median potassium concentration value calculated by solution for all participating laboratories in the interlaboratory-comparison program during 2009-10 for Norwegian Institute for Air Research (NILU), Shephard Analytical (SA), Ontario Ministry of Environment and Energy (MOEE), and New York State Department of Environmental Conservation (NYSDEC) laboratories. 
$\boldsymbol{A}$

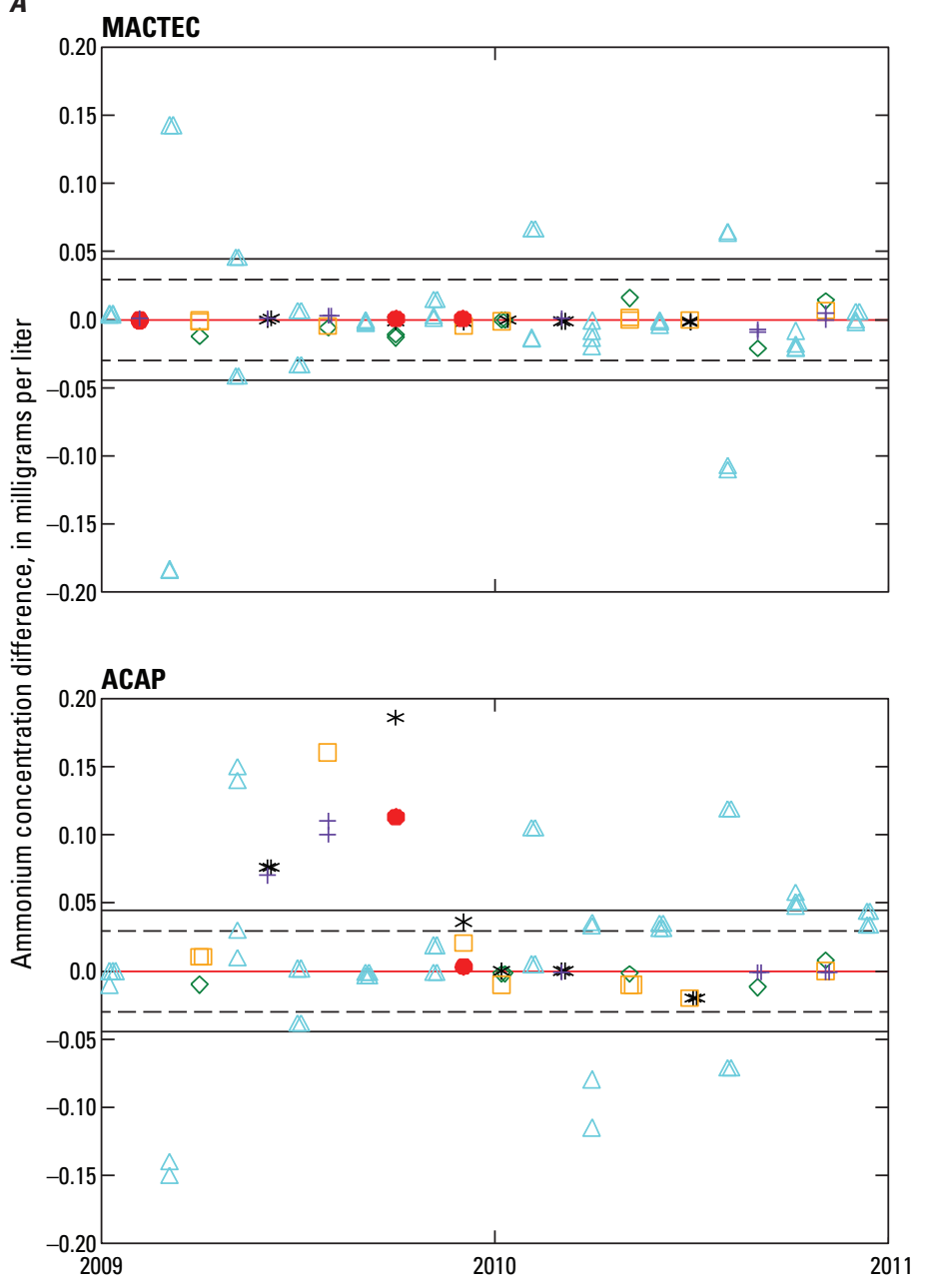

ECST

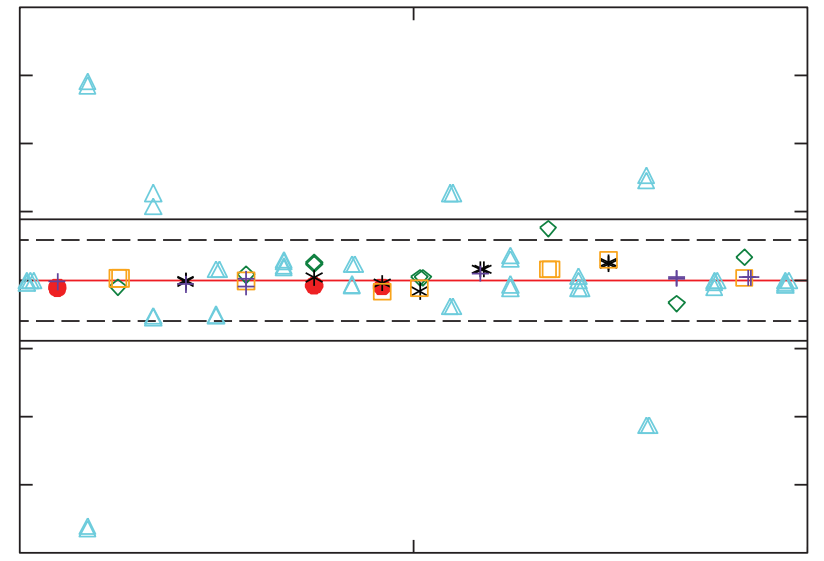

CAL

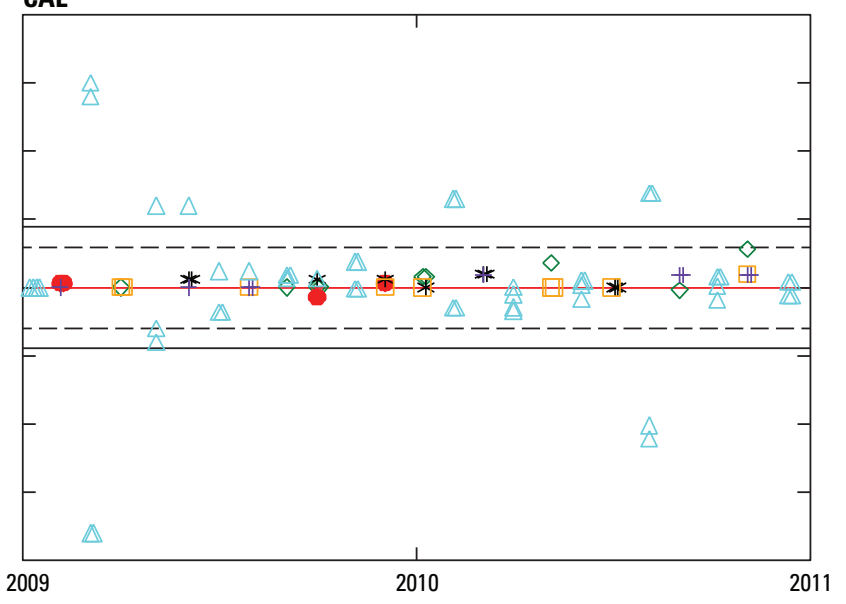

EXPLANATION

Control limit line -+3 and $-3 f$-pseudosigmas from zero difference

Warning limit line -+2 and $-2 f$-pseudosigmas from zero difference

Zero line

Solution-2009-2010 U.S. Geological Survey interlaboratory-comparison

program, table 4

SP1

SP17

SP21

SP3

SP97

Natural wet deposition-CALNAT

Laboratories:

MACTEC-MACTEC, Inc., Gainesville, Florida

ECST-Environment Canada Science \& Technology Branch (formerly MSC), Downsview, Ontario, Canada

ACAP_Asia Center for Air and Precipitation Research (formerly ADORC), Niigata-shi, Japan

CAL-Central Analytical Laboratory, Illinois State Water Survey, Champaign, Illinois

Figure 7. $A$, Difference between the measured ammonium concentration values and the median ammonium concentration value calculated by solution for all participating laboratories in the interlaboratory-comparison program during 2009-10 for MACTEC, Inc., Environment Canada Science and Technology Branch (ECST), Asia Center for Air and Precipitation Research (ACAP), and Central Analytical Laboratory (CAL) laboratories. 
$B$

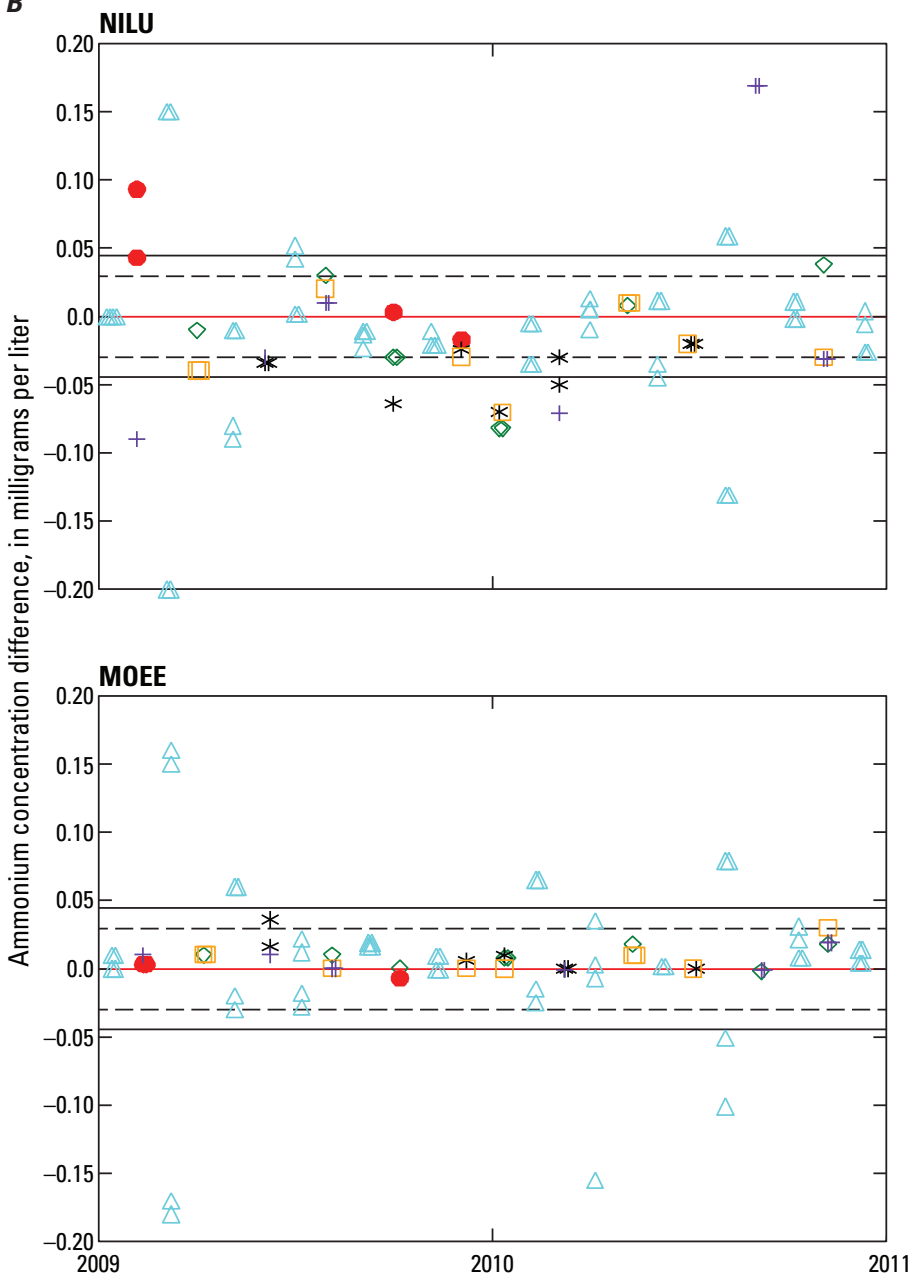

SA

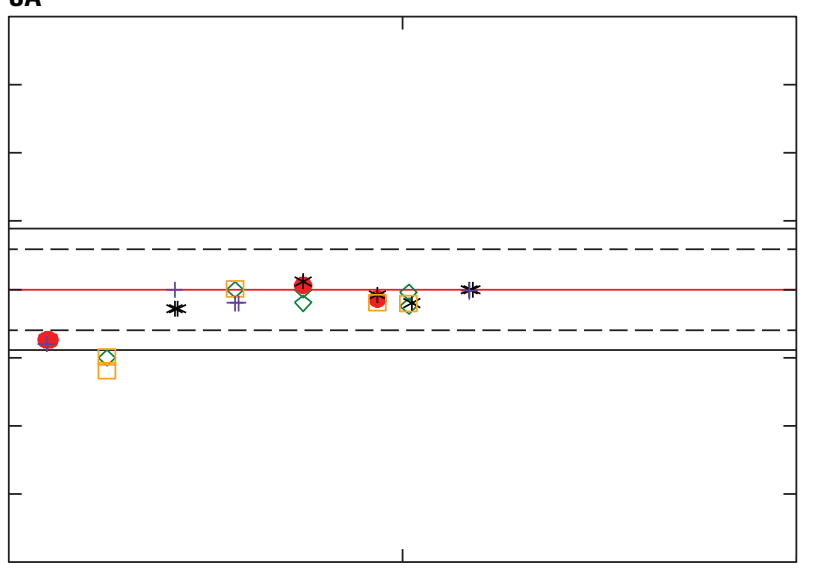

NYSDEC

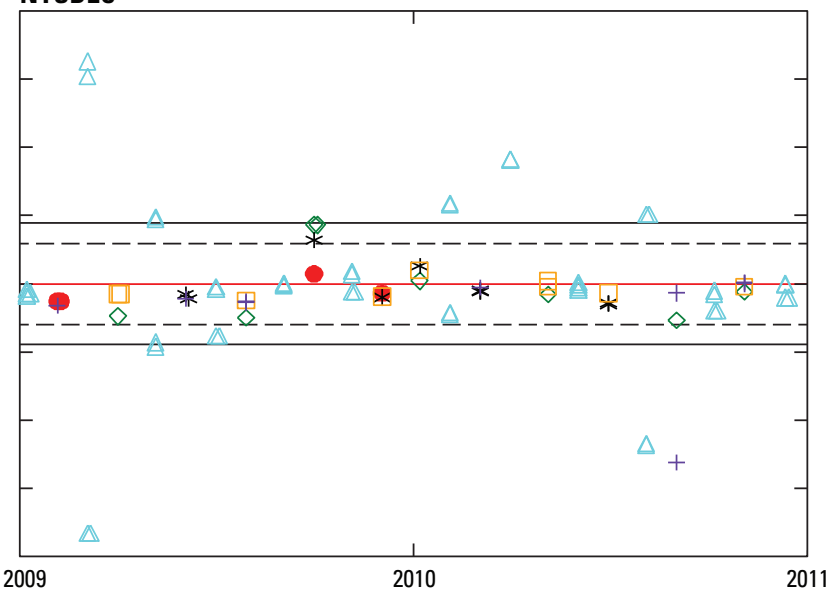

\section{EXPLANATION}

Control limit line -+3 and $-3 f$-pseudosigmas from zero difference

Warning limit line -+2 and $-2 f$-pseudosigmas from zero difference

Zero line

Solution-2009-2010 U.S. Geological Survey interlaboratory-comparison

program, table 4

$\diamond \quad$ SP1

- SP17

SP21

SP3

SP97

Natural wet deposition-CALNAT

Laboratories:

NILU-Norwegian Institute for Air Research, Kjeller, Norway

SA-Shepard Analytical, Simi Valley, California

MOEE-Ontario Ministry of Environment and Energy, Dorset Research Facility, Dorset, Ontario, Canada

NYSDEC_New York State Department of Environmental Conservation, Albany, New York

Figure 7. $\quad B$, Difference between the measured ammonium concentration values and the median ammonium concentration value calculated by solution for all participating laboratories in the interlaboratory-comparison program during 2009-10 for Norwegian Institute for Air Research (NILU), Shephard Analytical (SA), Ontario Ministry of Environment and Energy (MOEE), and New York State Department of Environmental Conservation (NYSDEC) laboratories. 
$\boldsymbol{A}$

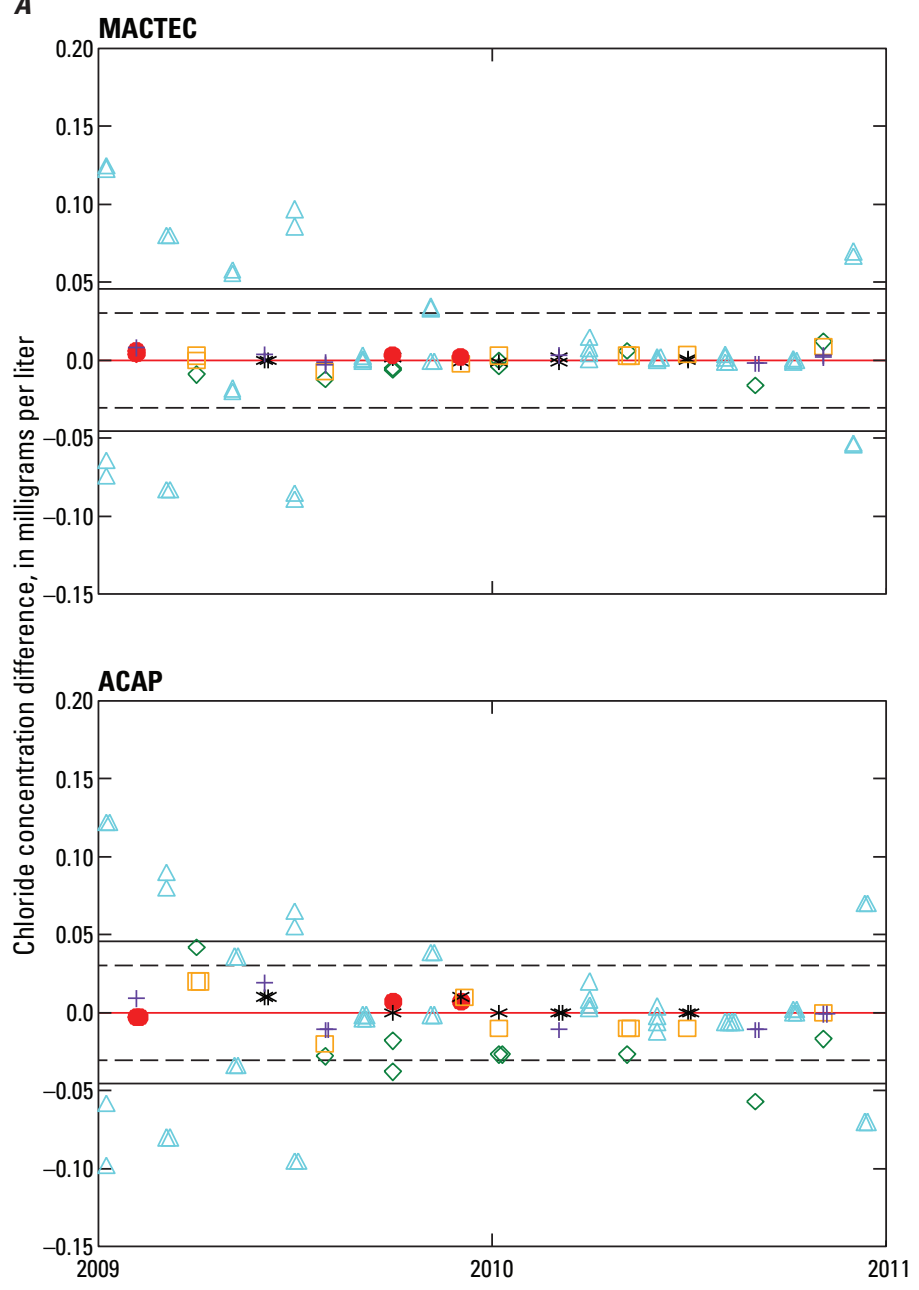

ECST

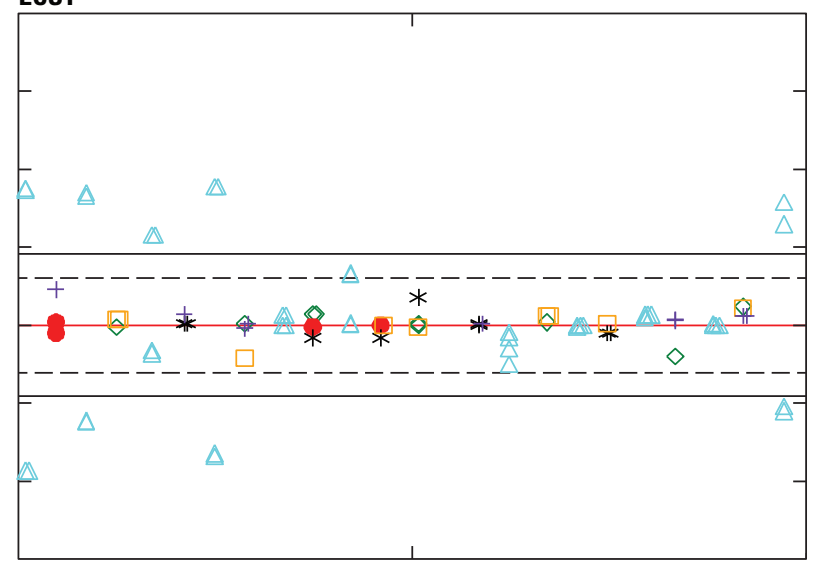

CAL

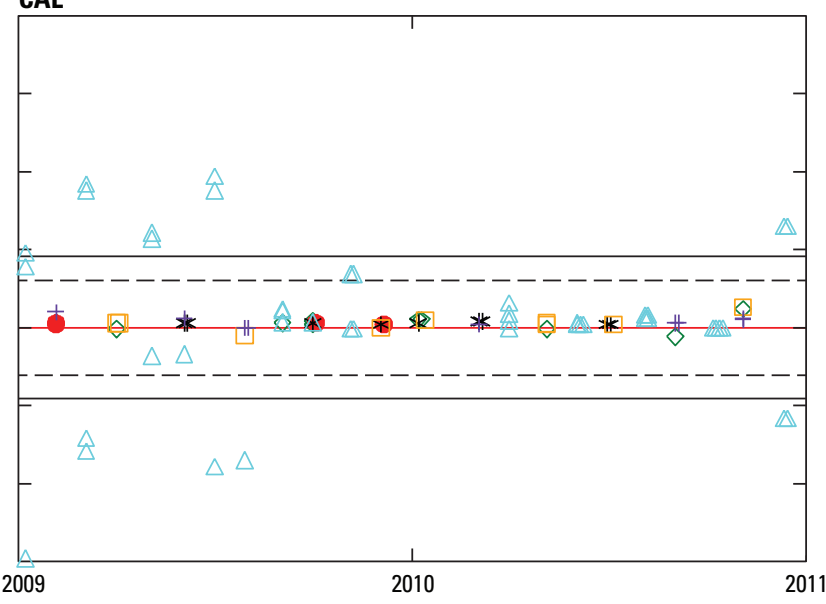

EXPLANATION

Control limit line -+3 and $-3 f$-pseudosigmas from zero difference Warning limit line -+2 and $-2 f$-pseudosigmas from zero difference Zero line

Solution-2009-2010 U.S. Geological Survey interlaboratory-comparison program, table 4

SP17

SP21

SP3

SP97

Natural wet deposition-CALNAT

Laboratories:

MACTEC-MACTEC, Inc., Gainesville, Florida

ECST-Environment Canada Science \& Technology Branch (formerly MSC), Downsview, Ontario, Canada

ACAP_ Asia Center for Air and Precipitation Research (formerly ADORC), Niigata-shi, Japan

CAL-Central Analytical Laboratory, Illinois State Water Survey, Champaign, Illinois

Figure 8. $\quad A$, Difference between the measured chloride concentration values and the median chloride concentration value calculated by solution for all participating laboratories in the interlaboratory-comparison program during 2009-10 for MACTEC, Inc., Environment Canada Science and Technology Branch (ECST), Asia Center for Air and Precipitation Research (ACAP), and Central Analytical Laboratory (CAL) laboratories. 
B

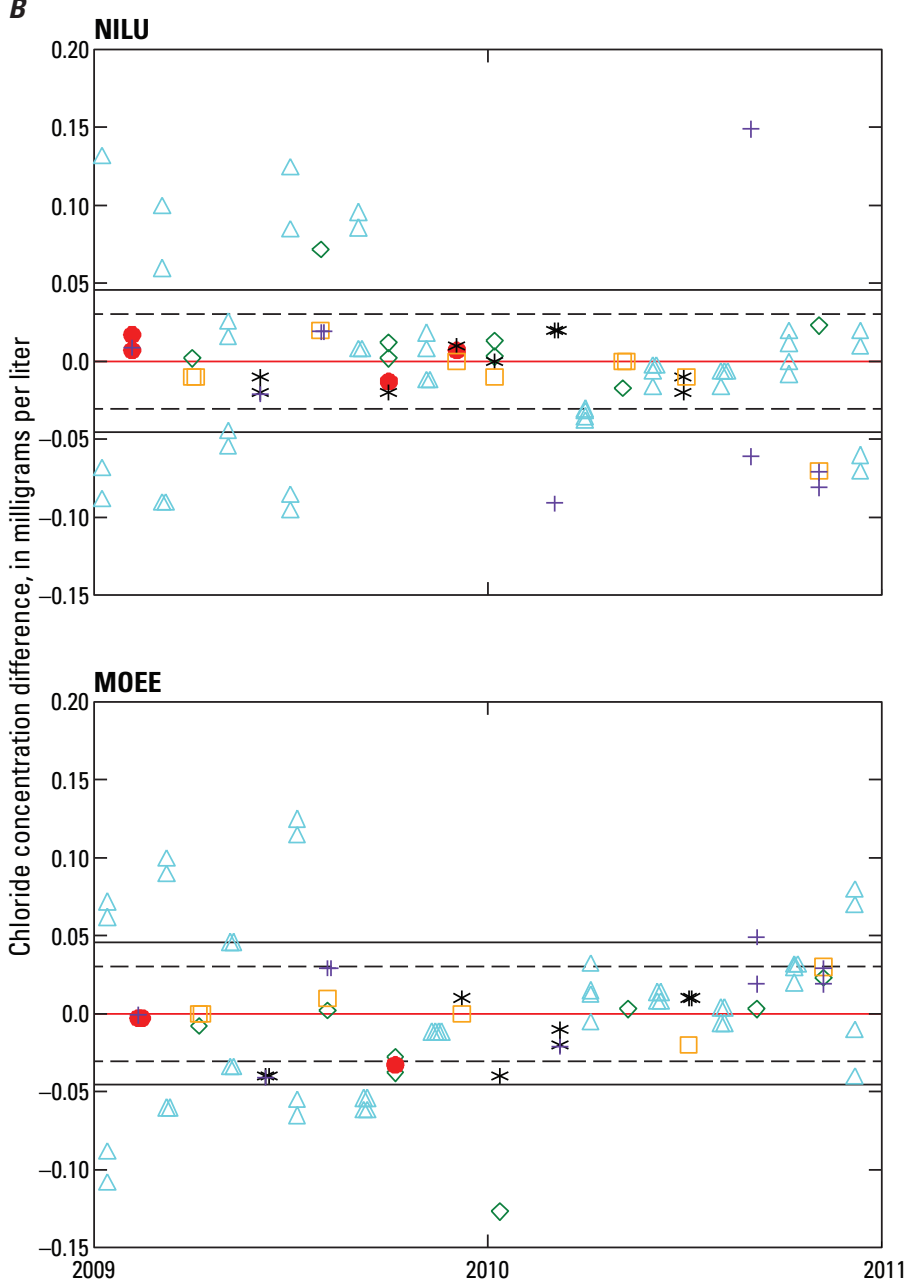

SA

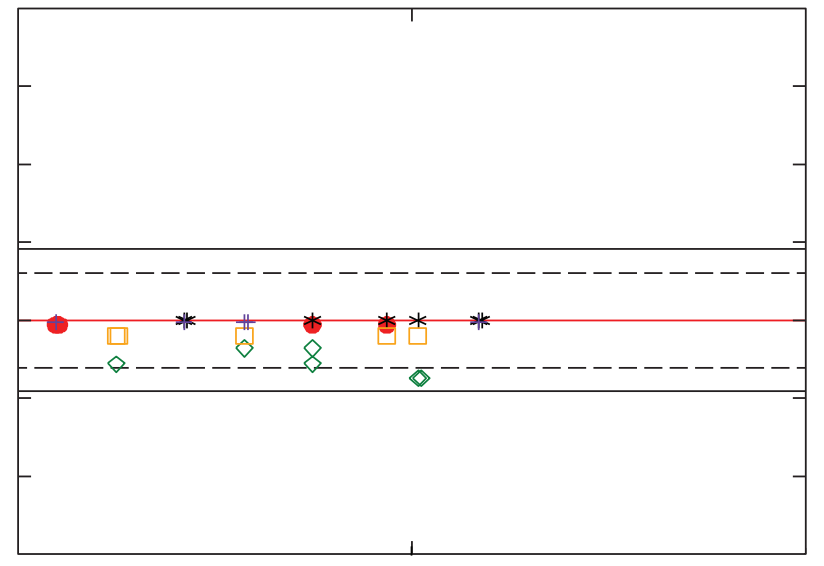

NYSDEC

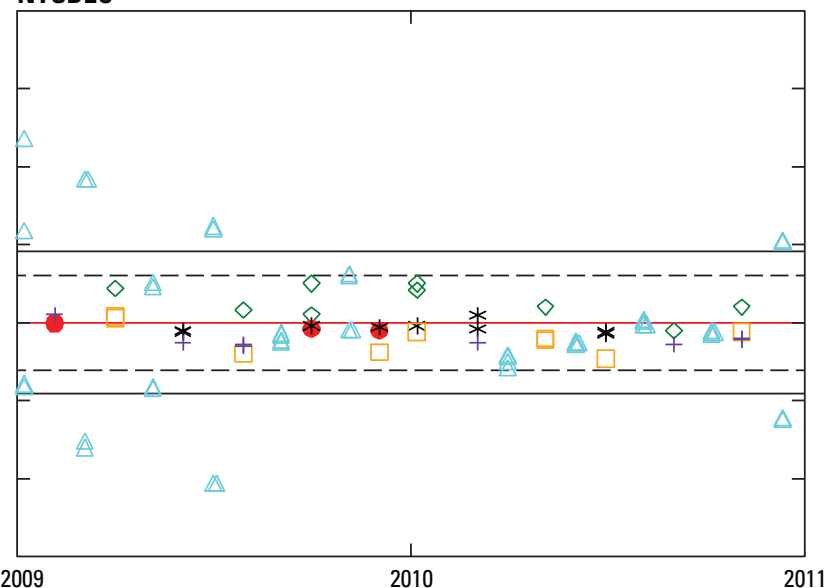

Year

\section{EXPLANATION}

Control limit line -+3 and $-3 f$-pseudosigmas from zero difference
$---\quad$ Warning limit line -+2 and $-2 f$-pseudosigmas from zero difference Zero line

Solution-2009-2010 U.S. Geological Survey interlaboratory-comparison

program, table 4

$\diamond \quad$ SP1

SP17

SP21

SP3

SP97

Natural wet deposition-CALNAT

Laboratories:

NILU—Norwegian Institute for Air Research, Kjeller, Norway

SA-Shepard Analytical, Simi Valley, California

MOEE- Ontario Ministry of Environment and Energy, Dorset Research Facility, Dorset, Ontario, Canada

NYSDEC—New York State Department of Environmental Conservation, Albany, New York

Figure 8. $B$, Difference between the measured chloride concentration values and the median chloride concentration value calculated by solution for all participating laboratories in the interlaboratory-comparison program during 2009-10 for Norwegian Institute for Air Research (NILU), Shephard Analytical (SA), Ontario Ministry of Environment and Energy (MOEE), and New York State Department of Environmental Conservation (NYSDEC) laboratories. 
A

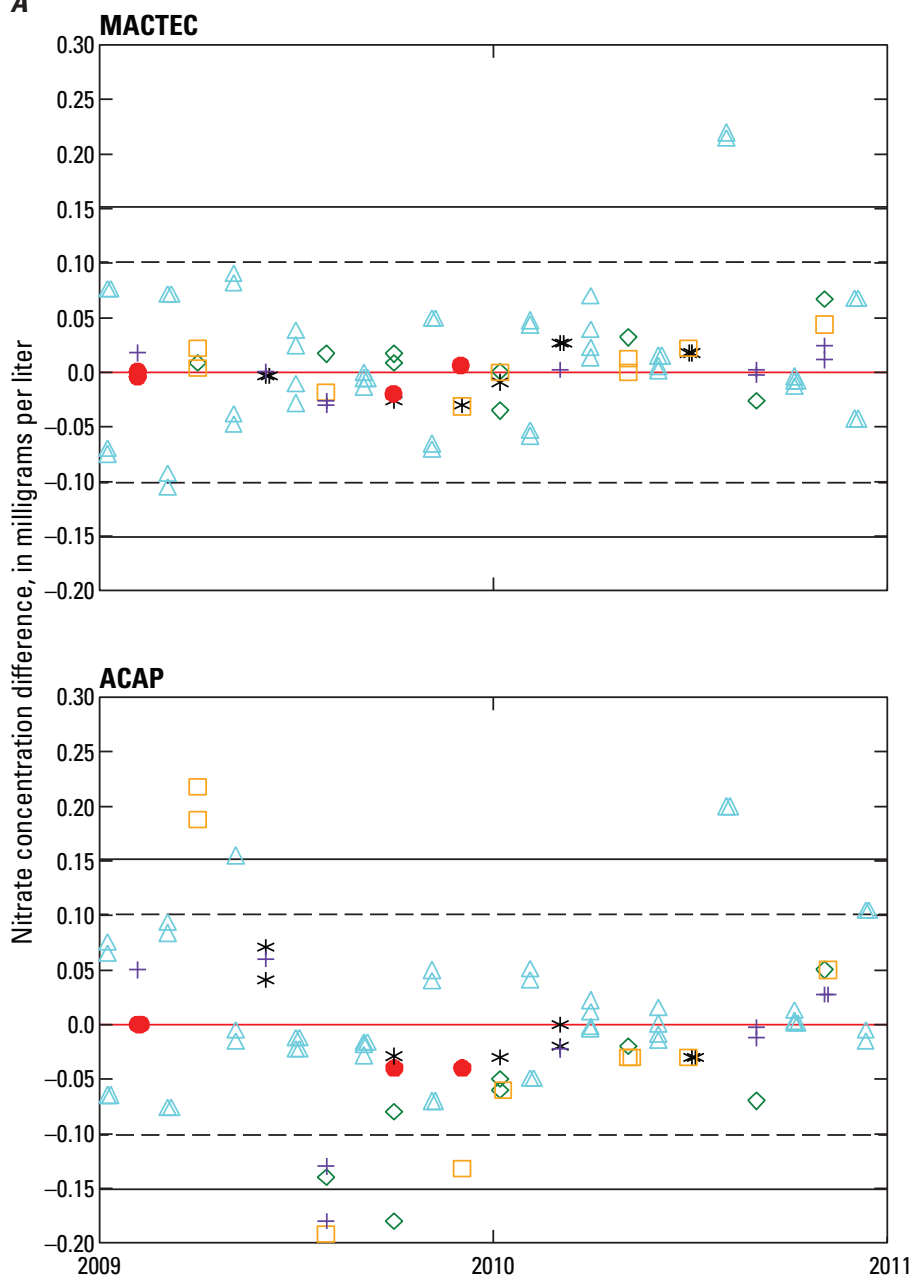

ECST

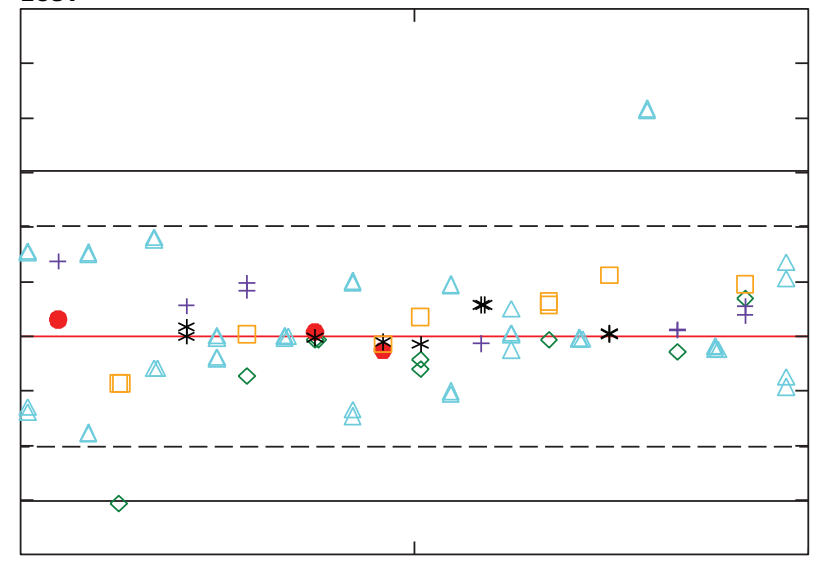

CAL

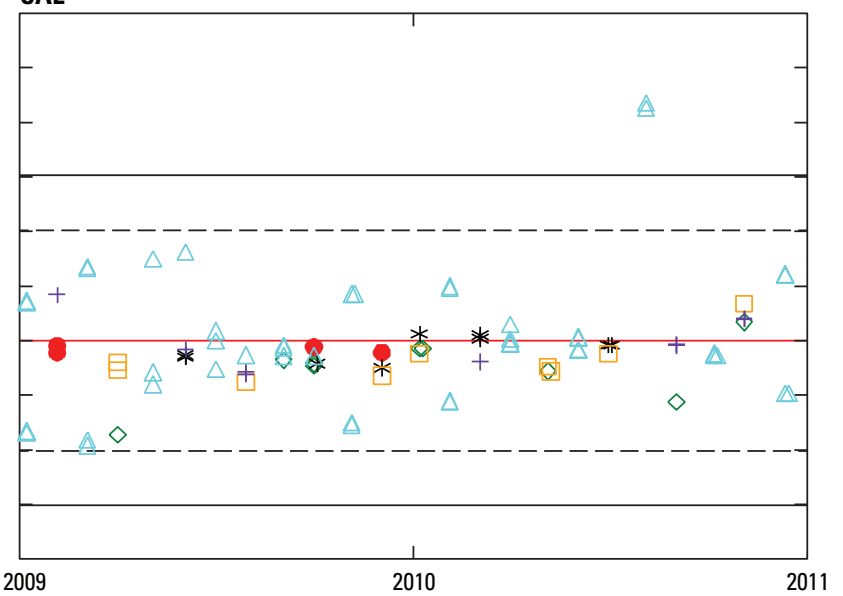

EXPLANATION

Control limit line- +3 and $-3 f$-pseudosigmas from zero difference

Warning limit line -+2 and $-2 f$-pseudosigmas from zero difference

Zero line

Solution-2009-2010 U.S. Geological Survey interlaboratory-comparison

program, table 4

SP1

SP17

SP21

SP3

SP97

Natural wet deposition-CALNAT

Laboratories:

MACTEC-MACTEC, Inc., Gainesville, Florida

ECST-Environment Canada Science \& Technology Branch (formerly MSC), Downsview, Ontario, Canada

ACAP_Asia Center for Air and Precipitation Research (formerly ADORC), Niigata-shi, Japan

CAL-Central Analytical Laboratory, Illinois State Water Survey, Champaign, Illinois

Figure 9. $A$, Difference between the measured nitrate concentration values and the median nitrate concentration value calculated by solution for all participating laboratories in the interlaboratory-comparison program during 2009-10 for MACTEC, Inc., Environment Canada Science and Technology Branch (ECST), Asia Center for Air and Precipitation Research (ACAP), and Central Analytical Laboratory (CAL) laboratories. 
$\boldsymbol{B}$

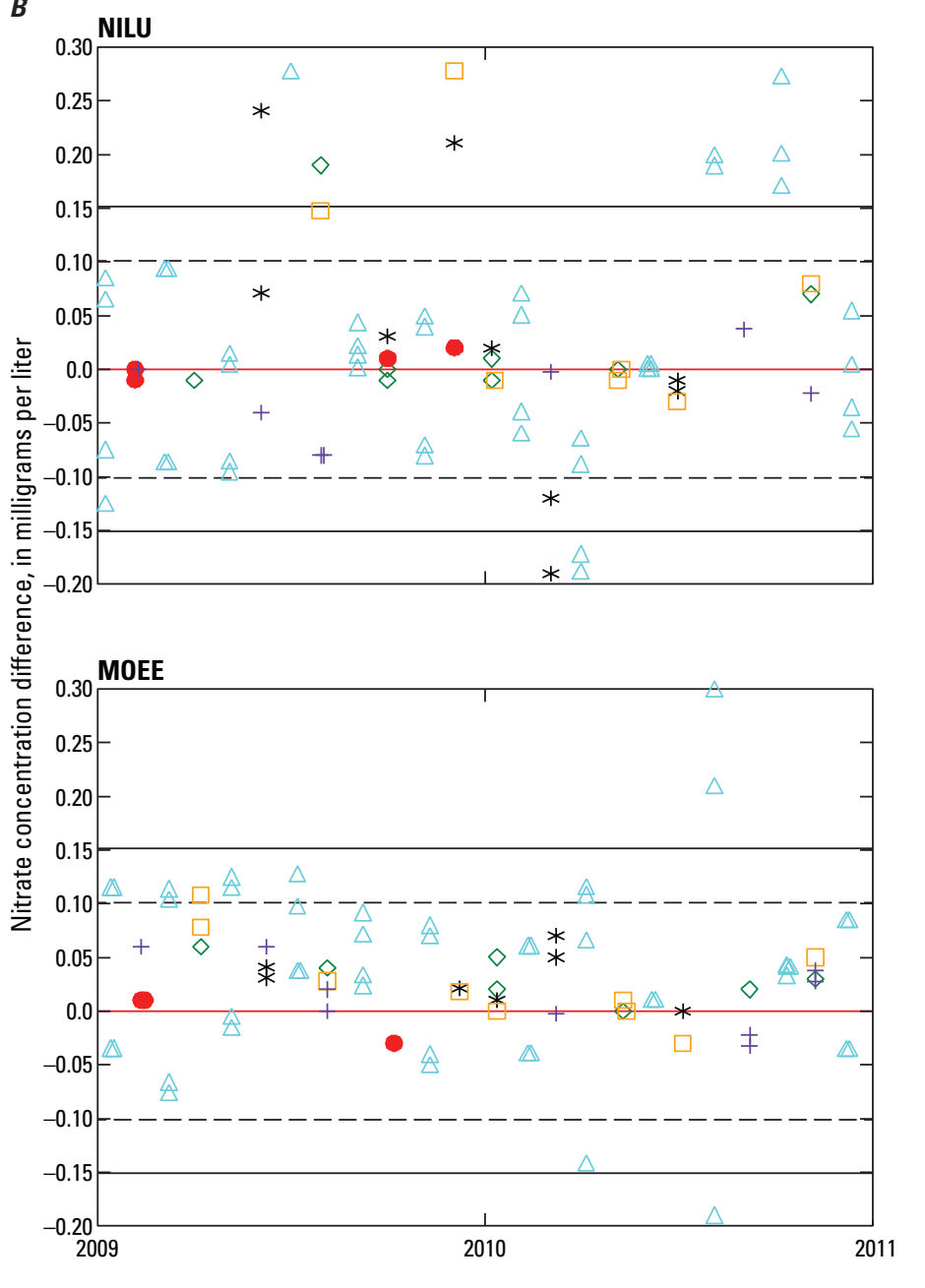

SA

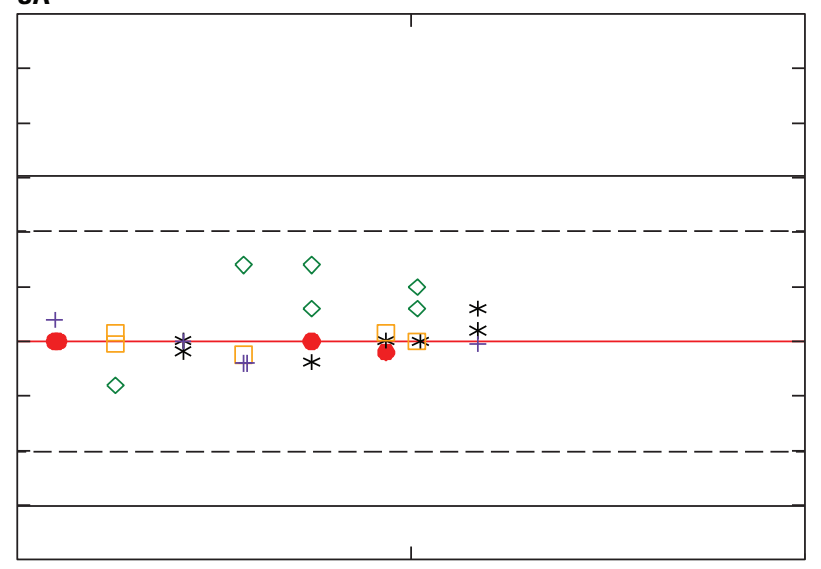

NYSDEC

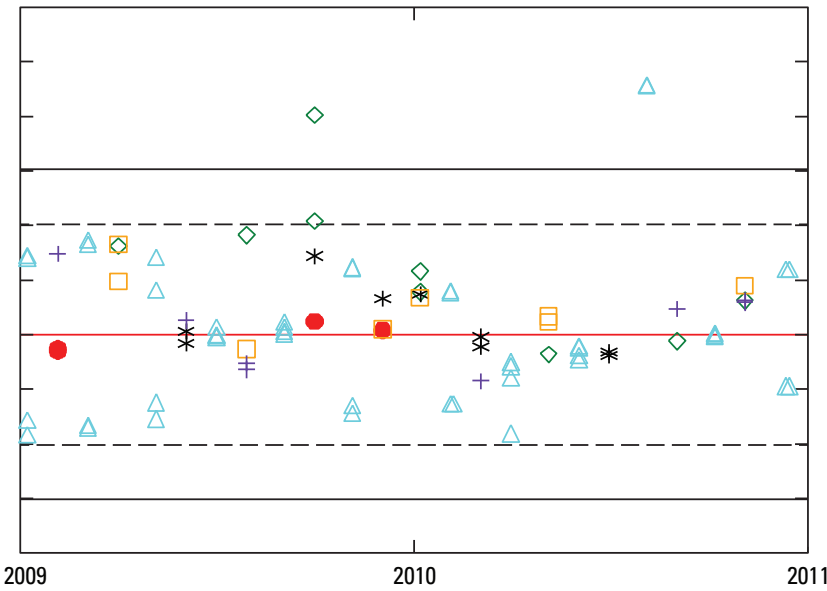

\section{EXPLANATION}

Control limit line- +3 and $-3 f$-pseudosigmas from zero difference

Warning limit line -+2 and $-2 f$-pseudosigmas from zero difference

Zero line

Solution-2009-2010 U.S. Geological Survey interlaboratory-comparison

program, table 4

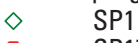

- SP17

SP21

SP3

SP97

Natural wet deposition-CALNAT

Laboratories:

NILU-Norwegian Institute for Air Research, Kjeller, Norway

SA-Shepard Analytical, Simi Valley, California

MOEE-Ontario Ministry of Environment and Energy, Dorset Research Facility, Dorset, Ontario, Canada

NYSDEC_New York State Department of Environmental Conservation, Albany, New York

Figure 9. $B$, Difference between the measured nitrate concentration values and the median nitrate concentration value calculated by solution for all participating laboratories in the interlaboratory-comparison program during 2009-10 for Norwegian Institute for Air Research (NILU), Shephard Analytical (SA), Ontario Ministry of Environment and Energy (MOEE), and New York State Department of Environmental Conservation (NYSDEC) laboratories. 
$\boldsymbol{A}$

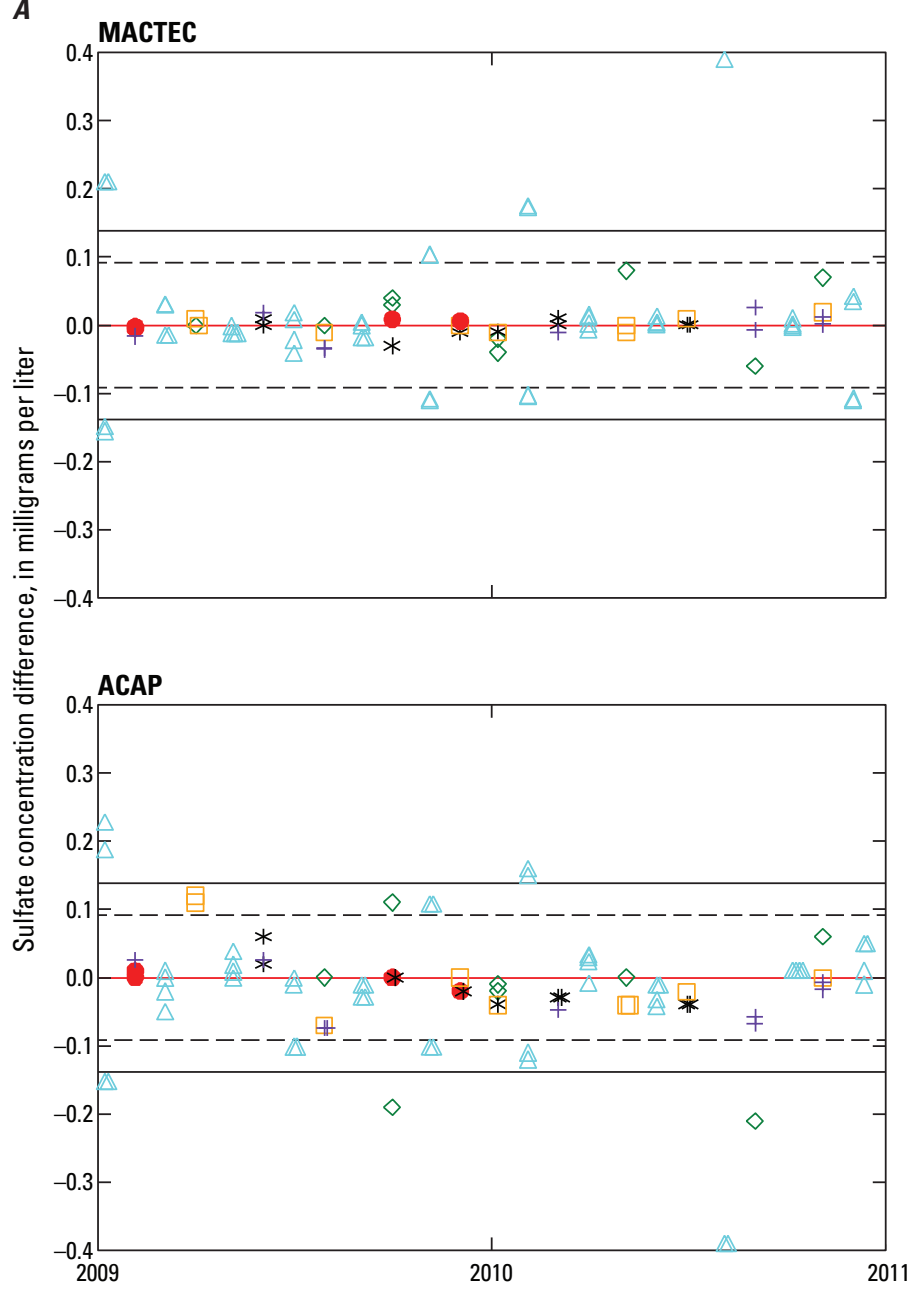

ECST

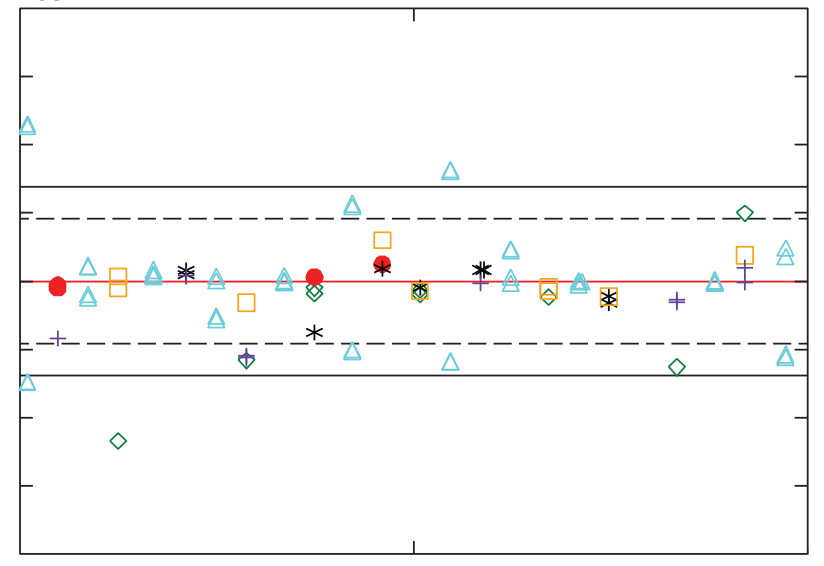

CAL

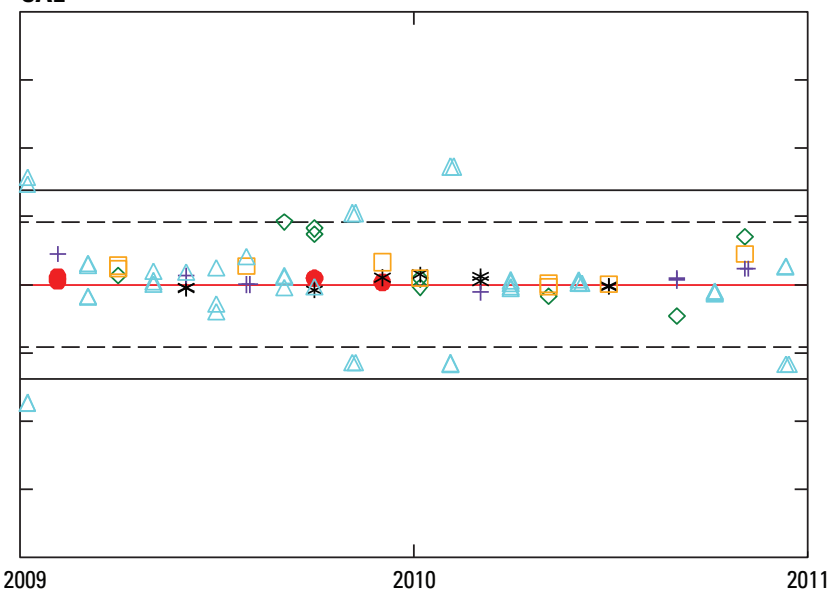

Year

\section{EXPLANATION}

Control limit line -+3 and $-3 f$-pseudosigmas from zero difference

Warning limit line -+2 and $-2 f$-pseudosigmas from zero difference

Zero line

Solution-2009-2010 U.S. Geological Survey interlaboratory-comparison

program, table 4

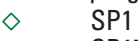

- SP17

SP21

SP3

SP97

Natural wet deposition-CALNAT

Laboratories:

MACTEC-MACTEC, Inc., Gainesville, Florida

ECST—Environment Canada Science \& Technology Branch (formerly MSC), Downsview, Ontario, Canada

ACAP_Asia Center for Air and Precipitation Research (formerly ADORC), Niigata-shi, Japan

CAL-Central Analytical Laboratory, Illinois State Water Survey, Champaign, Illinois

Figure 10. $A$, Difference between the measured sulfate concentration values and the median sulfate concentration value calculated by solution for all participating laboratories in the interlaboratory-comparison program during 2009-10 for MACTEC, Inc., Environment Canada Science and Technology Branch (ECST), Asia Center for Air and Precipitation Research (ACAP), and Central Analytical Laboratory (CAL) laboratories. 
$B$

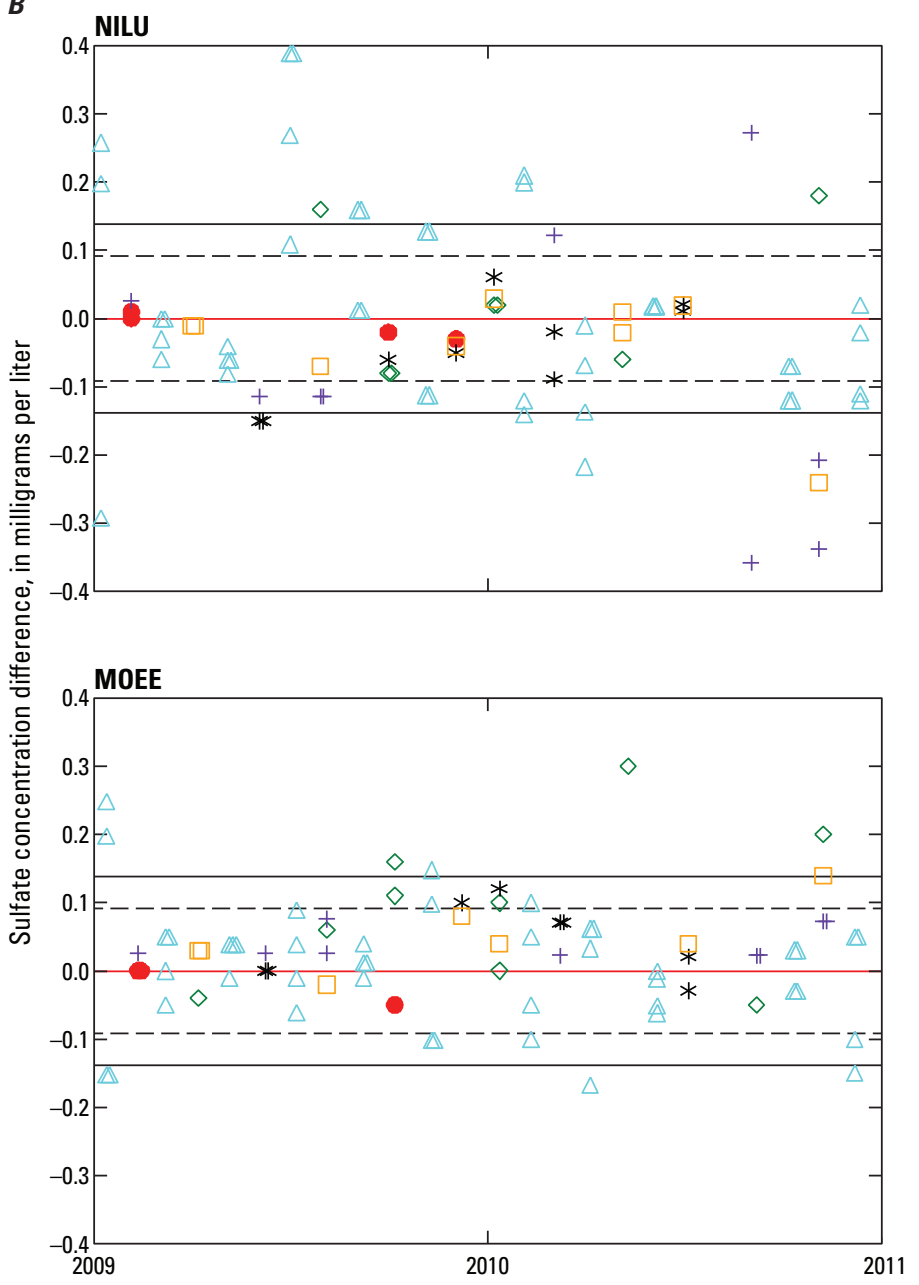

SA

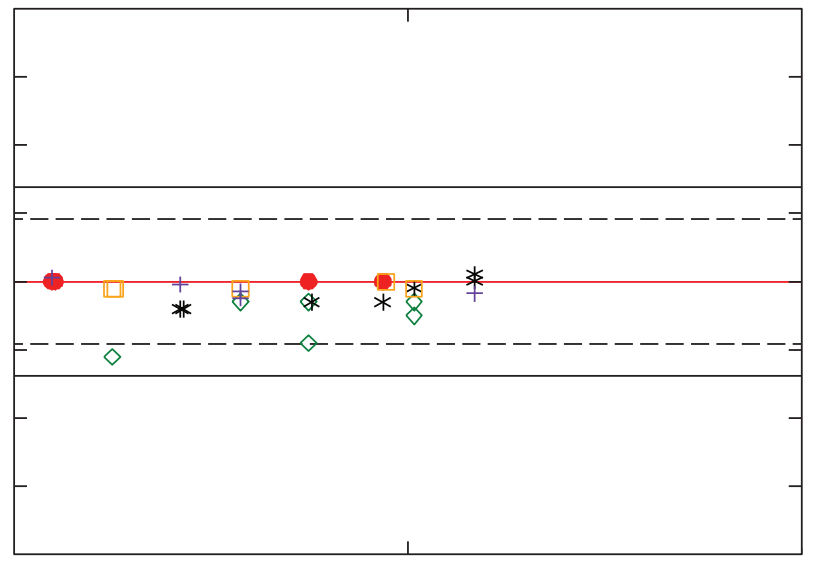

NYSDEC

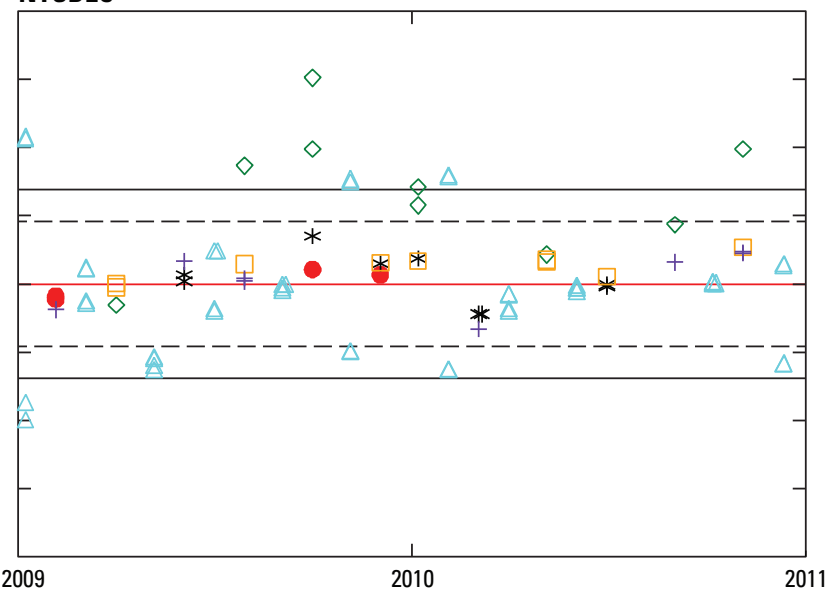

\section{EXPLANATION}

- Control limit line- +3 and $-3 f$-pseudosigmas from zero difference Warning limit line -+2 and $-2 f$-pseudosigmas from zero difference Zero line

Solution-2009-2010 U.S. Geological Survey interlaboratory-comparison

program, table 4

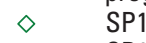

SP17

SP21

SP3

SP97

Natural wet deposition-CALNAT

Laboratories:

NILU-Norwegian Institute for Air Research, Kjeller, Norway

SA-Shepard Analytical, Simi Valley, California

MOEE- - Ontario Ministry of Environment and Energy, Dorset Research Facility, Dorset, Ontario, Canada

NYSDEC — New York State Department of Environmental Conservation, Albany, New York

Figure 10. $B$, Difference between the measured sulfate concentration values and the median sulfate concentration value calculated by solution for all participating laboratories in the interlaboratory-comparison program during 2009-10 for Norwegian Institute for Air Research (NILU), Shephard Analytical (SA), Ontario Ministry of Environment and Energy (MOEE), and New York State Department of Environmental Conservation (NYSDEC) laboratories. 
$\boldsymbol{A}$

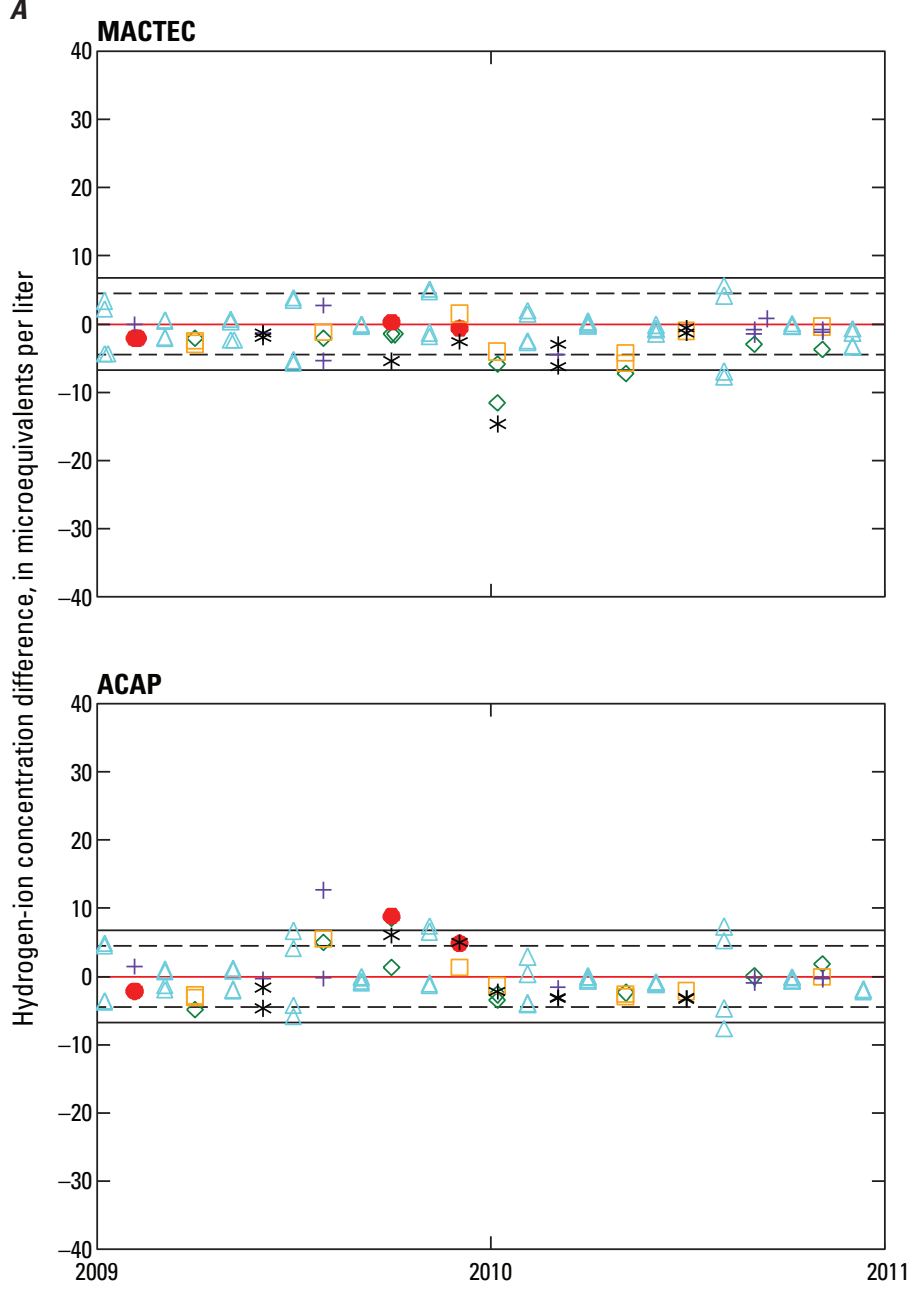

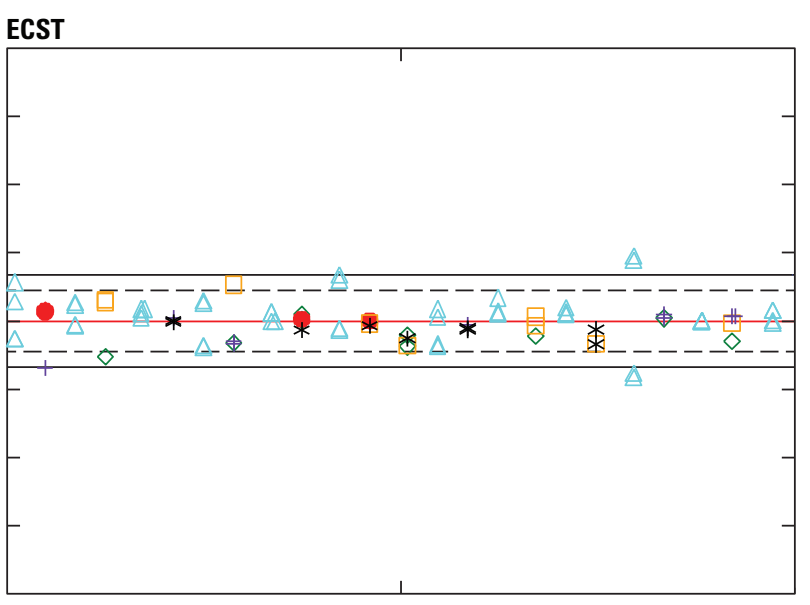

CAL

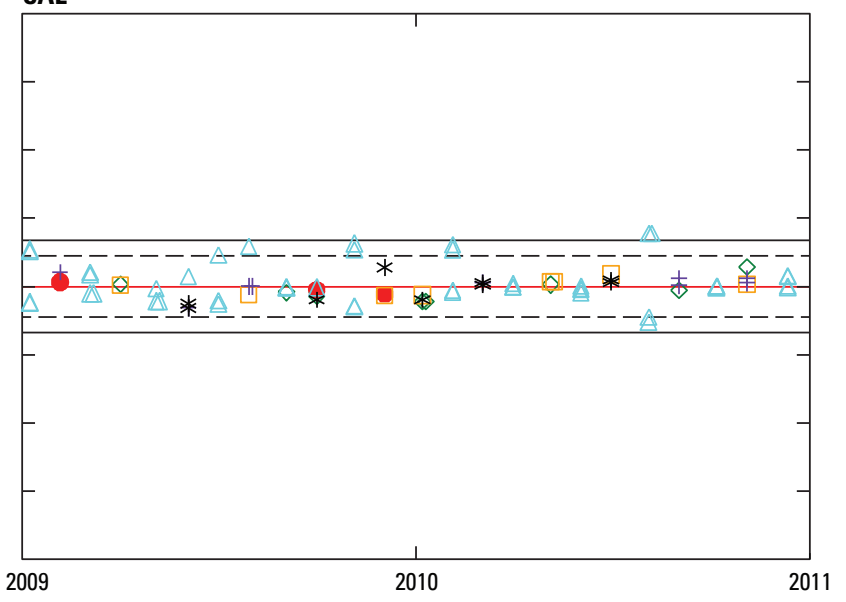

\section{EXPLANATION}

Control limit line -+3 and $-3 f$-pseudosigmas from zero difference

Warning limit line -+2 and $-2 f$-pseudosigmas from zero difference

Zero line

Solution-2009-2010 U.S. Geological Survey interlaboratory-comparison

program, table 4

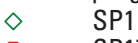

- SP17

SP21

SP3

SP97

Natural wet deposition-CALNAT

Laboratories:

MACTEC_MACTEC, Inc., Gainesville, Florida

ECST_Environment Canada Science \& Technology Branch (formerly MSC), Downsview, Ontario, Canada

ACAP_Asia Center for Air and Precipitation Research (formerly ADORC), Niigata-shi, Japan

CAL_Central Analytical Laboratory, Illinois State Water Survey, Champaign, Illinois

Figure 11. A, Difference between the measured hydrogen-ion concentration values and the median hydrogen-ion concentration value calculated by solution for all participating laboratories in the interlaboratory-comparison program during 2009-10 for MACTEC, Inc., Environment Canada Science and Technology Branch (ECST), Asia Center for Air and Precipitation Research (ACAP), and Central Analytical Laboratory (CAL) laboratories. 
B

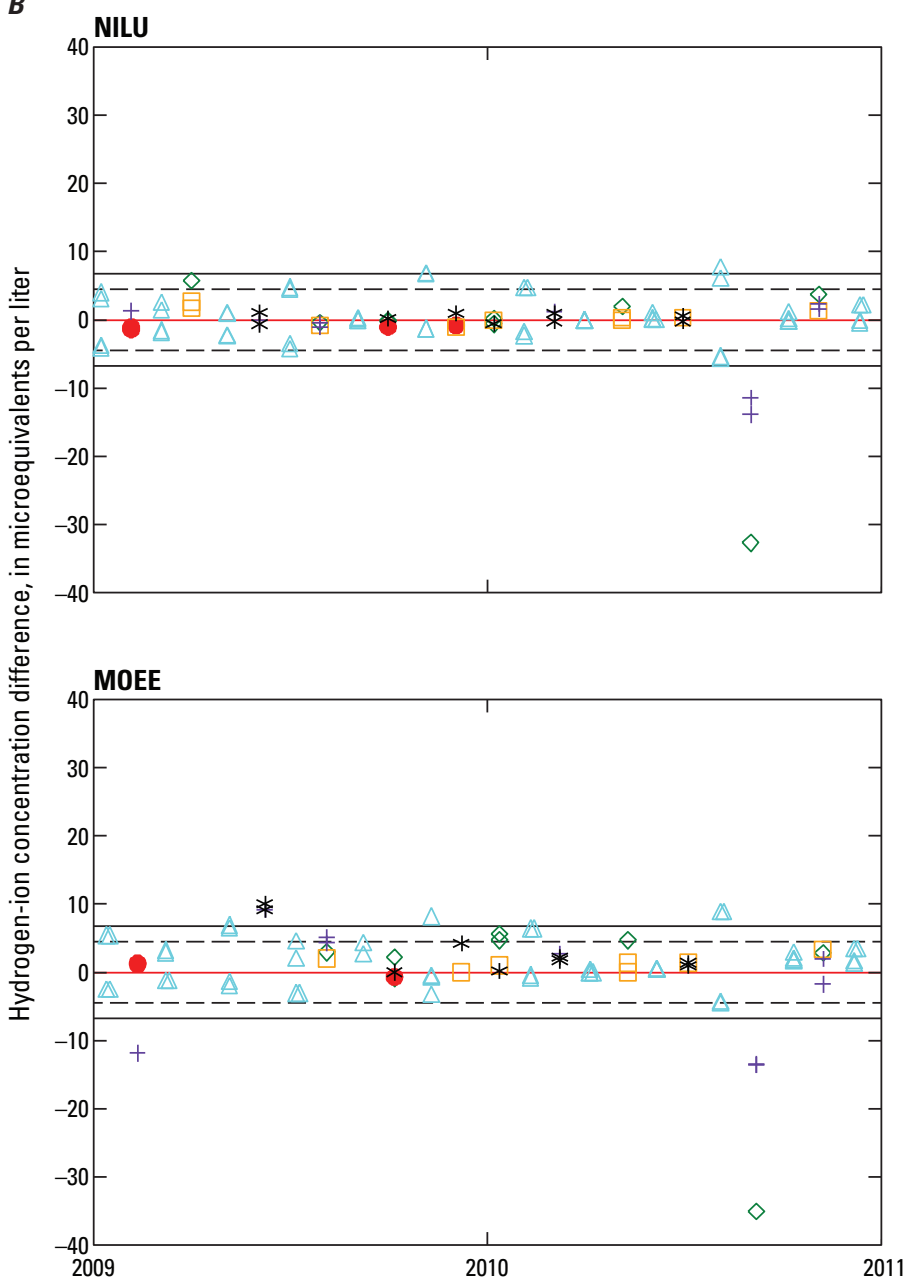

SA

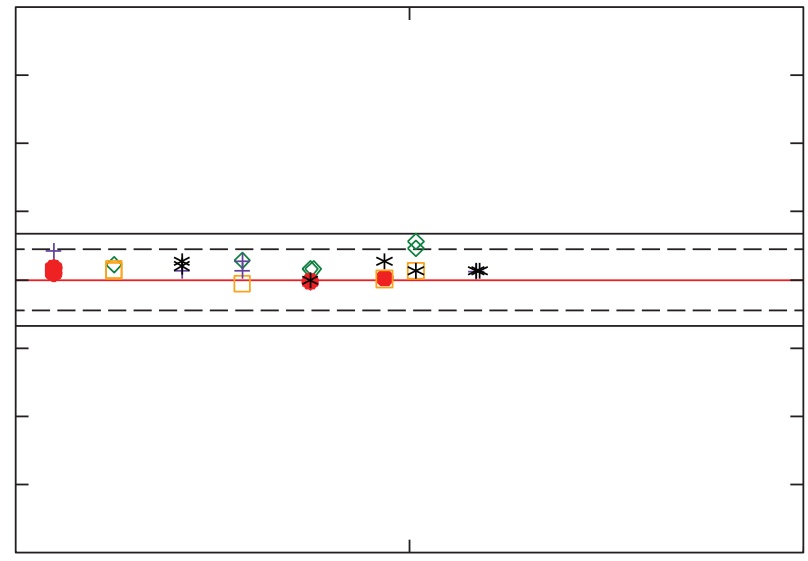

NYSDEC

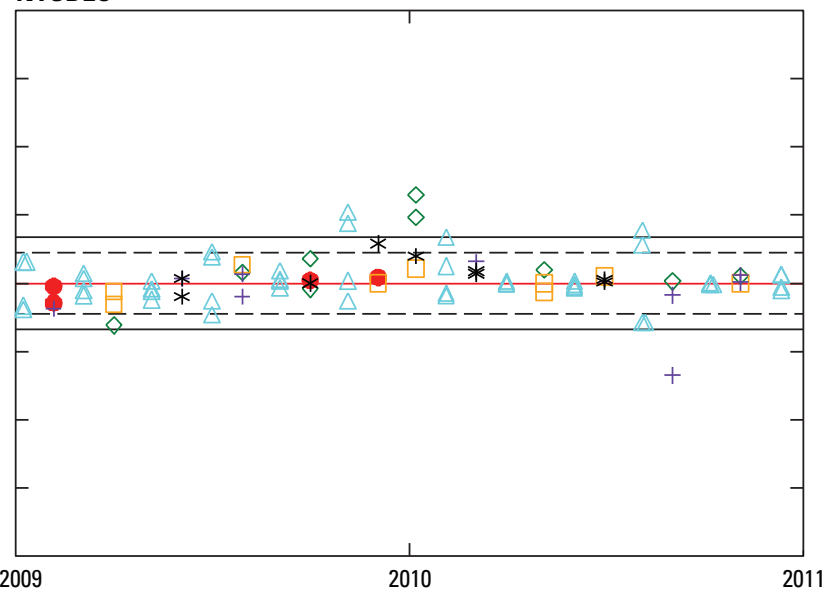

\section{EXPLANATION}

Control limit line- +3 and $-3 f$-pseudosigmas from zero difference

--- Warning limit line -+2 and $-2 f$-pseudosigmas from zero difference

Zero line

Solution-2009-2010 U.S. Geological Survey interlaboratory-comparison

program, table 4

$\diamond \quad \mathrm{SP} 1$

SP17

SP21

$\mathrm{SP} 3$

SP97

Natural wet deposition-CALNAT

Laboratories:

NILU—Norwegian Institute for Air Research, Kjeller, Norway

SA-Shepard Analytical, Simi Valley, California

MOEE-Ontario Ministry of Environment and Energy, Dorset Research Facility, Dorset, Ontario, Canada

NYSDEC — New York State Department of Environmental Conservation, Albany, New York

Figure 11. $B$, Difference between the measured hydrogen-ion concentration values and the median hydrogen-ion concentration value calculated by solution for all participating laboratories in the interlaboratory-comparison program during 2009-10 for Norwegian Institute for Air Research (NILU), Shephard Analytical (SA), Ontario Ministry of Environment and Energy (MOEE), and New York State Department of Environmental Conservation (NYSDEC) laboratories. 
$\boldsymbol{A}$

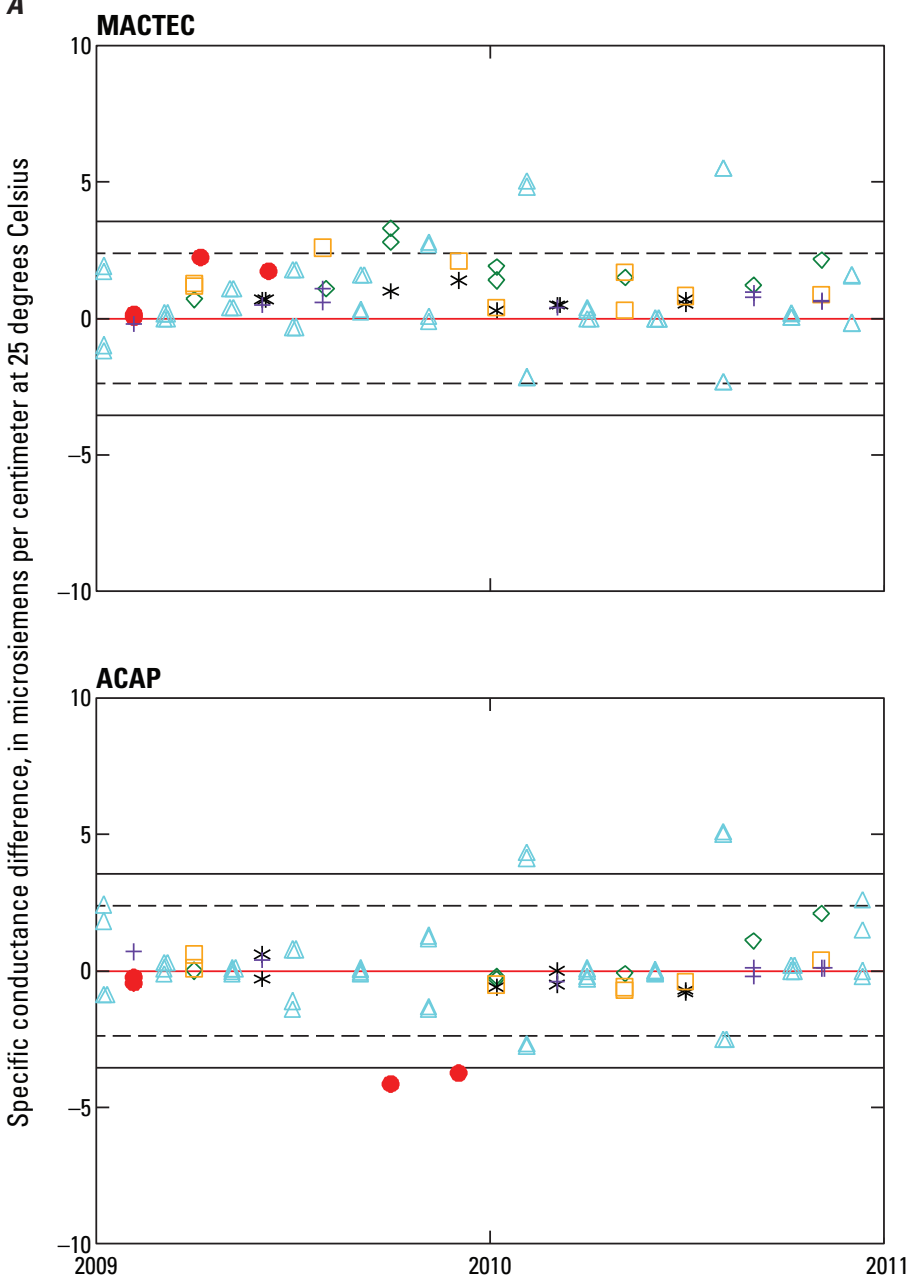

ECST does not measure specific conductance.

CAL

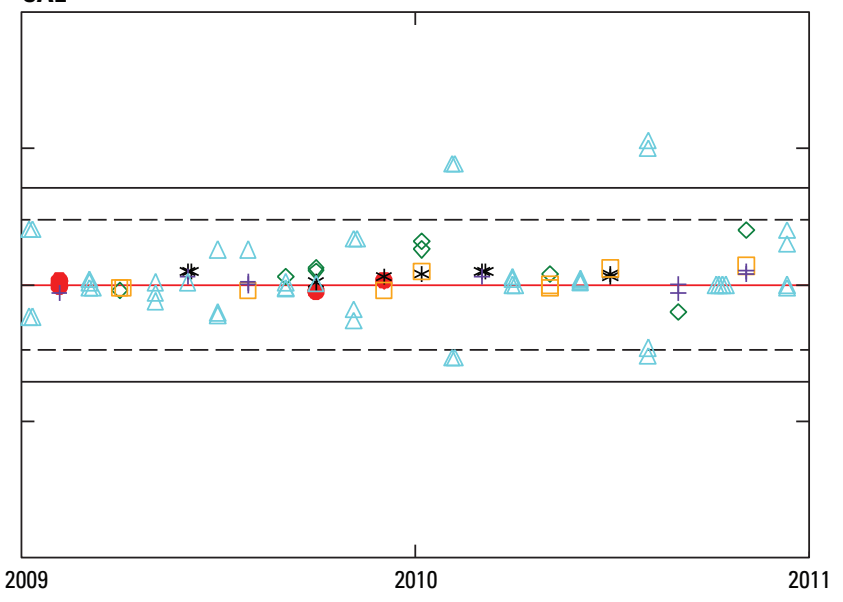

Year

EXPLANATION

Control limit line -+3 and $-3 f$-pseudosigmas from zero difference

Warning limit line -+2 and $-2 f$-pseudosigmas from zero difference

Zero line

Solution-2009-2010 U.S. Geological Survey interlaboratory-comparison

program, table 4

$\diamond \quad$ SP1

SP17

SP21

SP3

SP97

Natural wet deposition-CALNAT

Laboratories:

MACTEC-MACTEC, Inc., Gainesville, Florida

ECST-Environment Canada Science \& Technology Branch (formerly MSC), Downsview, Ontario, Canada

ACAP_Asia Center for Air and Precipitation Research (formerly ADORC), Niigata-shi, Japan

CAL-Central Analytical Laboratory, Illinois State Water Survey, Champaign, Illinois

Figure 12. A, Difference between the measured specific conductance values and the median specific conductance value calculated by solution for all participating laboratories in the interlaboratory-comparison program during 2009-10 for MACTEC, Inc., Environment Canada Science and Technology Branch (ECST), Asia Center for Air and Precipitation Research (ACAP), and Central Analytical Laboratory (CAL) laboratories. 
$B$

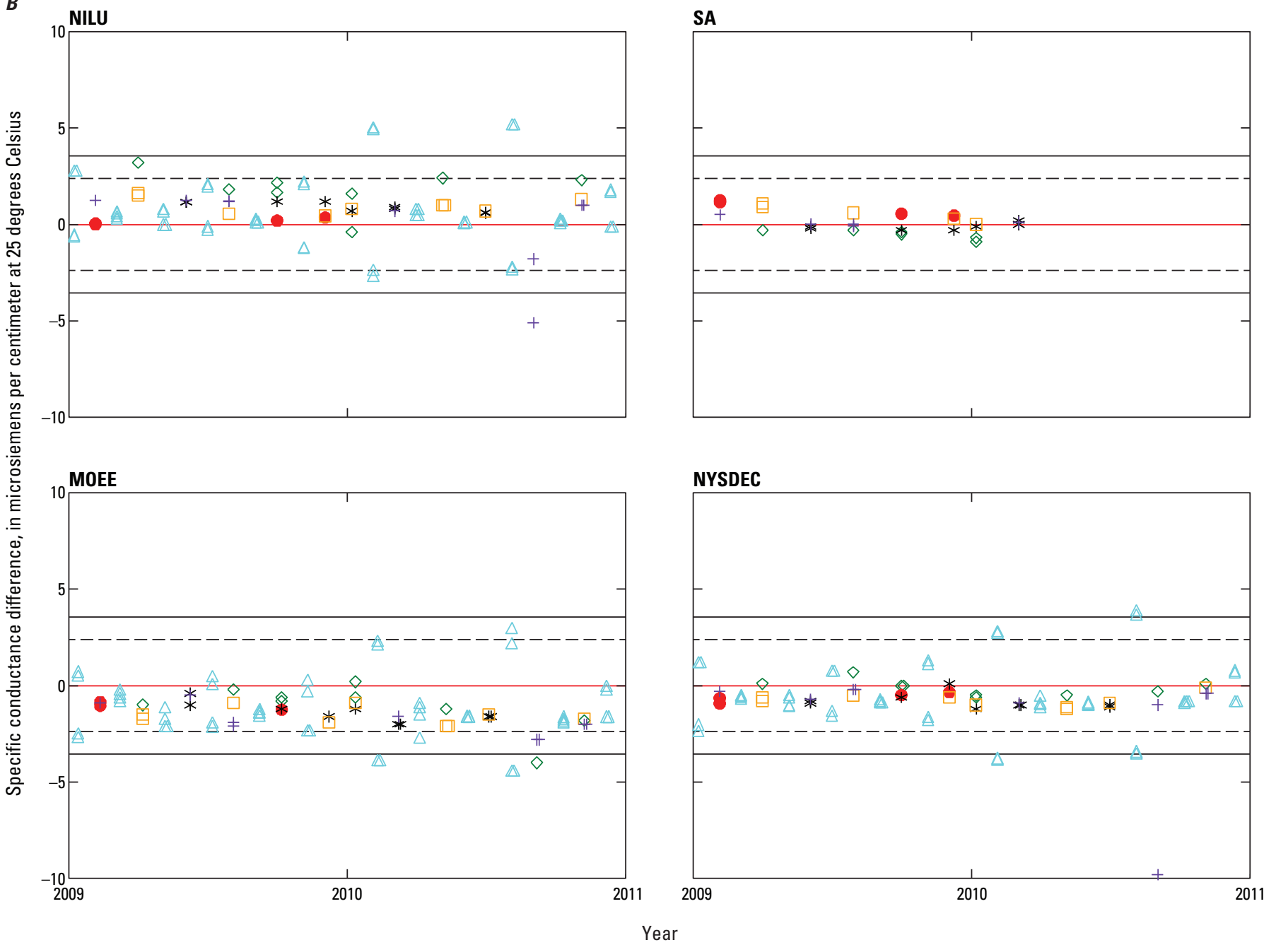

EXPLANATION

Control limit line -+3 and $-3 f$-pseudosigmas from zero difference

Warning limit line -+2 and $-2 f$-pseudosigmas from zero difference

Zero line

Solution-2009-2010 U.S. Geological Survey interlaboratory-comparison

program, table 4

$\diamond \quad$ SP1

- SP17

SP21

SP3

SP97

Natural wet deposition-CALNAT

Laboratories:

NILU—Norwegian Institute for Air Research, Kjeller, Norway

SA-Shepard Analytical, Simi Valley, California

MOEE-Ontario Ministry of Environment and Energy, Dorset Research Facility, Dorset, Ontario, Canada

NYSDEC — New York State Department of Environmental Conservation, Albany, New York

Figure 12. $B$, Difference between the measured specific conductance values and the median specific conductance value calculated by solution for all participating laboratories in the interlaboratory-comparison program during 2009-10 for Norwegian Institute for Air Research (NILU), Shephard Analytical (SA), Ontario Ministry of Environment and Energy (MOEE), and New York State Department of Environmental Conservation (NYSDEC) laboratories. 

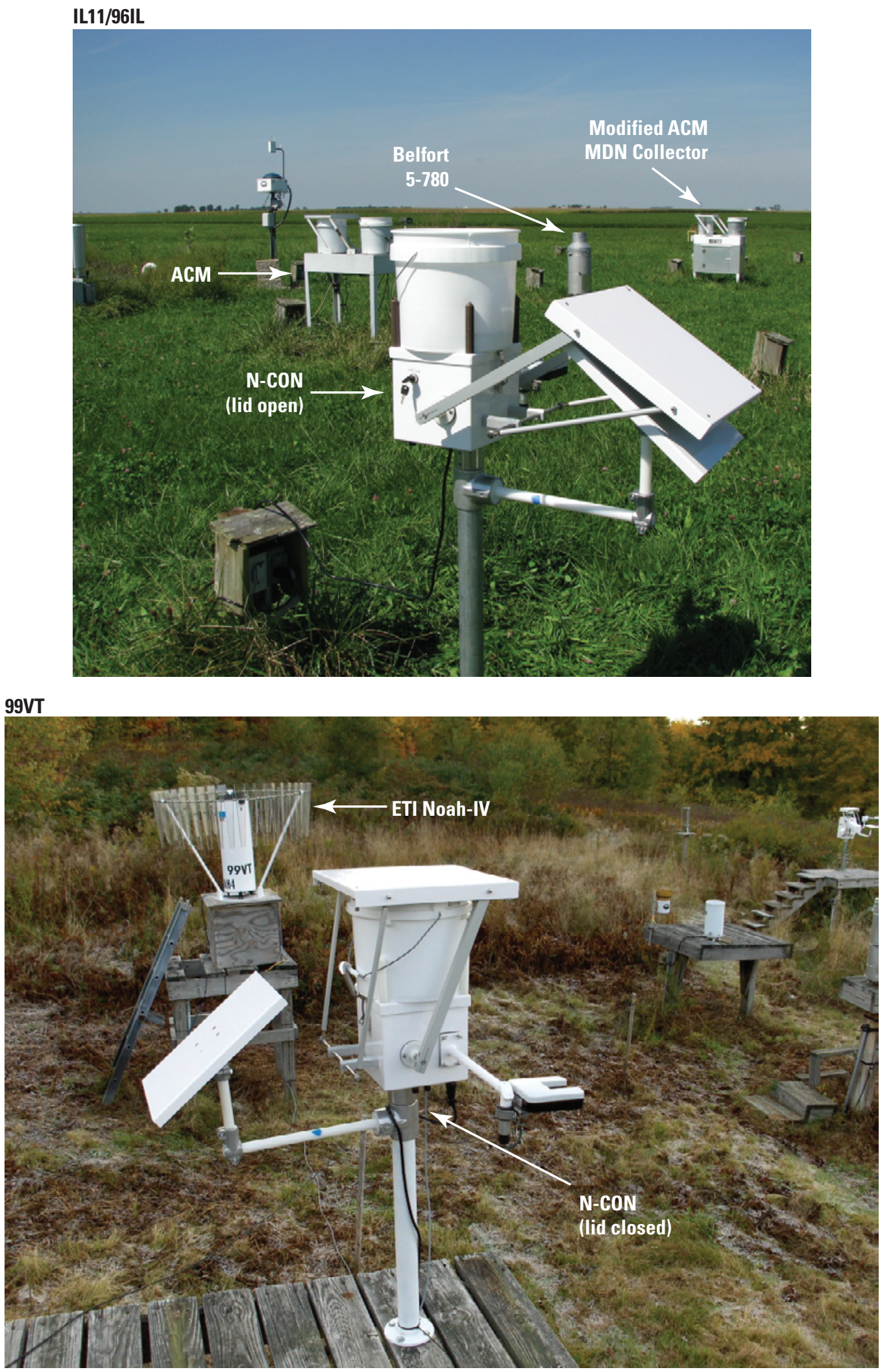

Figure 13. Co-located N-CON collector 96IL and ACM collector IL11 (background-left) at Bondville, Illinois (top) and N-CON collector 99VT at Underhill, Vermont (bottom). 


\section{Co-located-Sampler Program}

The co-located-sampler program was established in October 1988 to assess the overall variability of the data imparted by the physical wet-deposition-monitoring equipment and sample processing system used by NTN. Included in this estimate of NTN precision is the variability from the point of sample collection through laboratory analysis and quality control (Gordon, 1999). Since 1988, co-located sites have been operated on a water-year basis (October 1 to September 30) every year except 1994 (Gordon, 1999; Wetherbee and others, 2005a). Nilles and others (1991) provide a detailed description of the co-located-sampler program. Recent modification of the goals and objectives of the program are described by Wetherbee and others (2009 and 2010).

A 5-yr co-located study concluded at site VT99 (Underhill, Vermont (Vt.)) at the end of water year 2010. Results from the first $3 \mathrm{yr}$ are documented in Wetherbee and others (2009 and 2010). During water year 2009, the VT99 site consisted of the original mechanical Belfort Model 5-780 rain gage and Aerochem Metrics Model 301 collector. The co-located 99VT site included an ETI Noah-IV electronic rain gage and a prototype bucket collector manufactured by N-CON Systems, Inc., Crawford, Georgia (N-CON, fig. 13). In October 2009, a new ETI Noah-IV rain gage replaced the existing Belfort gage to become the rain gage of record for VT99.

Co-located ACM and N-CON collectors were operated at IL11 (Bondville, Ill.) during water years 2009-10 (fig. 13). The co-located collector was assigned ID 96IL. IL11 has two rain gages: (1) an OTT Pluvio-N rain gage, which began collecting data on August 23, 2007 and (2) the original Belfort Model 5-780 rain gage operated through March 17, 2009.

Co-located pairs of ACM collectors were operated during water year 2010 at sites CO98 (original site) and CO89 (co-located site) at Loch Vale, Rocky Mountain National Park. CO98 was retrofit with an ETI Noah-IV gage in 2008, and the original Belfort rain gage operated until August 9, 2010 when it was removed from the site, and a second ETI Noah-IV gage was installed in its place for site CO89.

Co-located OTT Pluvio-N electronic recording rain gages were operated by the USGS Indiana Water Science Center at MDN site IN26 (Fort Harrison State Park, Indiana) during water year 2009. The co-located OTT Pluvio-N gage was assigned ID 26IN. This co-located study was done to quantify variability of the OTT Pluvio-N gage, 7 of which were put into service at NADP sites: IL11, IN20, IN21, IN26, IN28, IN34, and NJ30. OTT discontinued the Pluvio-N model and replaced it with the Pluvio2 model, which NADP adopted as a suitable replacement in 2009 (National Atmospheric Deposition Program, 2009).

At each co-located site, instruments were installed such that they were exposed to identical conditions. Snow platforms, rain-gage shielding, and other accessories were duplicated. Proper operation of each set of co-located equipment, per manufacturer specifications and NADP criteria, was verified by USGS before starting sample collection at the co-located sites (Dossett and Bowersox, 1999). Co-located sites were operated using identical field and laboratory sample collection and analysis procedures.

\section{Precipitation-Gage Comparisons}

\section{Sites VT99/99VT}

During the 5-yr study period (water years 2005-10), the original Belfort 5-780 rain gage operated at VT99 until water year 2010 when it was replaced with an ETI Noah-IV rain gage. In water year 2005, the co-located rain gage at 99VT was also a Belfort 5-780 until water year 2007, when it was switched to an ETI Noah-IV rain gage. Cumulative precipitation-depth data indicate no appreciable bias between the two Belfort 5-780 rain gages in water years 2005-06 (fig. 14). Median weekly precipitation-depth absolute differences between the co-located Belfort gages ranged from 0.3 to 1.8 percent (table 8 ). During water years 2007-09, the Noah-IV rain gages recorded median weekly precipitation depths that were 5.7 to 8.0 percent greater than the original, co-located Belfort gage depths (table 8). A shift in the cumulative precipitation data is observed for the co-located gages at the point when the Belfort gage was replaced by a second Noah-IV gage (fig. 14). During water year 2010, the colocated, identical Noah-IV gages recorded annual precipitation depths within 0.6 percent median absolute percent difference (table 8).

Daily precipitation-depth data for the co-located Noah-IV rain gages at VT99/99VT are plotted against each other in figure 15, and they follow the 1:1 line with three points plotting above the line. Weeks with frozen versus liquid or mixed phase precipitation were determined by weekly maximum rain gage datalogger temperature. Significant $(\alpha=0.05)$ bias per the sign test was observed between the VT99 and 99VT Noah-IV rain gages for liquid precipitation $(p=0.033)$ but not for frozen precipitation $(p=0.093)$. However, the R-square values for regression of the daily precipitation depths are 0.99 and 0.97 for liquid and frozen precipitation, respectively, with estimated slope parameters of 1.0 ( $p$ less than 0.0001 ) for both cases. Therefore, the bias between the original and co-located Noah-IV gages at VT99/99VT is statistically significant but practically negligible.

\section{Sites C098/C089}

Belfort gage annual precipitation-depths were approximately 6 percent lower than those measured by the ETI Noah-IV rain gage during water years 2008-09 at CO98/CO89 (table 8). Median absolute percent difference between the co-located Noah-IV gages was 2.2 percent during water year 2010. Variability in paired weekly precipitation depth data for co-located Noah-IV rain gages at CO98/CO89 is similar to variability observed in the VT99/99VT data (fig. 15). For CO98/CO89, at least 11 points with increased bias are observed in figure 15 at precipitation depths of 0.6 inches or less, at least four of which are for weeks with frozen precipitation. 
Table 8. Precipitation depths measured at co-located rain gages; National Atmospheric Deposition Program sites VT99/99VT, C098/C089, IL11, and IN26/26IN, during water years 2005-10.

[Belfort, Belfort Model 5-780 mechanical precipitation gage; Noah-IV, ETI Noah-IV electronic precipitation gage; OTT Pluvio-N, OTT Pluvio type NADP electronic precipitation gage]

\begin{tabular}{|c|c|c|c|c|}
\hline Water Year & Original gage & Co-located gage & $\begin{array}{l}\text { Median weekly } \\
\text { original-minus- } \\
\text { co-located difference } \\
\text { (percent) }\end{array}$ & $\begin{array}{c}\text { Concurrent record } \\
\text { available for both } \\
\text { gages (days) }\end{array}$ \\
\hline & VT99 & 99VT & & \\
\hline 2005 & Belfort & Belfort & -1.8 & 343 \\
\hline 2006 & Belfort & Belfort & -0.3 & 287 \\
\hline 2007 & Belfort & Belfort/Noah-IV & 0.6 & 294 \\
\hline 2008 & Belfort & Noah-IV & -5.7 & 350 \\
\hline 2009 & Belfort & Noah-IV & -8.0 & 350 \\
\hline \multirow[t]{2}{*}{2010} & Noah-IV & Noah-IV & -0.6 & 203 \\
\hline & C098 & CO89 & & \\
\hline 2008 & Belfort & Noah-IV & -6.4 & 322 \\
\hline 2009 & Belfort & Noah-IV & -6.2 & 326 \\
\hline \multirow[t]{2}{*}{2010} & Noah-IV & Noah-IV & 2.2 & 355 \\
\hline & $\mathrm{IL}_{11}{ }^{1}$ & $\mathrm{IL}_{1} 1^{2}$ & & \\
\hline \multirow[t]{2}{*}{2009} & Belfort & OTT Pluvio-N & -4.1 & 133 \\
\hline & IN26 & $26 I N$ & & \\
\hline 2010 & OTT Pluvio-N & OTT Pluvio-N & 1.7 & 187 \\
\hline
\end{tabular}

${ }^{1}$ IL11 original Belfort gage operated at IL11.

${ }^{2}$ IL11 new OTT Pluvio-N installed to become new gage of record.

Statistically significant $(\alpha=0.05)$ bias between the CO98 and CO89 Noah-IV rain gages was indicated by the sign test for both liquid $(p=0.003)$ and frozen $(\mathrm{p}=0.008)$ precipitation. However, the R-square values for regression of the daily precipitation depths are 0.97 and 0.99 for liquid and frozen precipitation, respectively, with estimated slope parameters of 0.98 and 0.99 ( $p$ less than 0.0001), respectively. Therefore, the bias between the original and co-located Noah-IV gages at CO98 and CO89 is statistically significant but practically negligible.

\section{Sites IN26/26IN}

Weekly precipitation depths for IN26/26IN co-located OTT Pluvio-N rain gages are shown in figure 16. Points that plot farthest from the 1:1 line in figure 16 are for weeks with frozen precipitation. The median absolute percent difference in the cumulative precipitation depths obtained over 187 days was 1.7 percent, which is similar to the differences observed for the paired Noah-IV gages (table 8). Statistically significant $(\alpha=0.05)$ bias per the sign test was not indicated between the co-located OTT Pluvio-N rain gages for liquid precipitation $(p=0.243)$, but bias was indicated for frozen precipitation $(p=0.016)$. Regression of the co-located IN26/26IN paired precipitation depths yielded adjusted R-square values of 0.97 and estimated slopes of 1.0 ( $p$-value less than 0.001 ) for both liquid and frozen precipitation. Therefore, the bias between the original and co-located OTT Pluvio-N rain gages at IN26 and 26IN is statistically significant but practically negligible.

\section{Site IL11}

The OTT Pluvio-N rain gage measured approximately 4.1 percent (median) greater precipitation depth than the Belfort rain gage at IL11 during 2009 (table 8). This comparison is based on a relatively short period of record of 133 days.

\section{Precipitation Collector Comparison}

Precipitation chemistry data from co-located sites were analyzed for differences between samples from each collector. Data for wet-deposition samples with volumes greater than $35 \mathrm{~mL}$ were used (Mark Rhodes, Illinois State Water Survey, electronic commun., March 2010 and March 2011), which are identified in the NADP database by a laboratorytype code "W" to indicate that the samples were of sufficient volume for analysis and did not require dilution. Explanatory information for the NADP data is available on the NADP web site: $h t t p: / / n a d p . s w s . u i u c . e d u /$. Samples requiring dilution are inherently prone to a greater error component. Samples identified as contaminated with debris, bird droppings, insects, dirt or soot particles, or mishandled were eliminated from statistical analysis. 


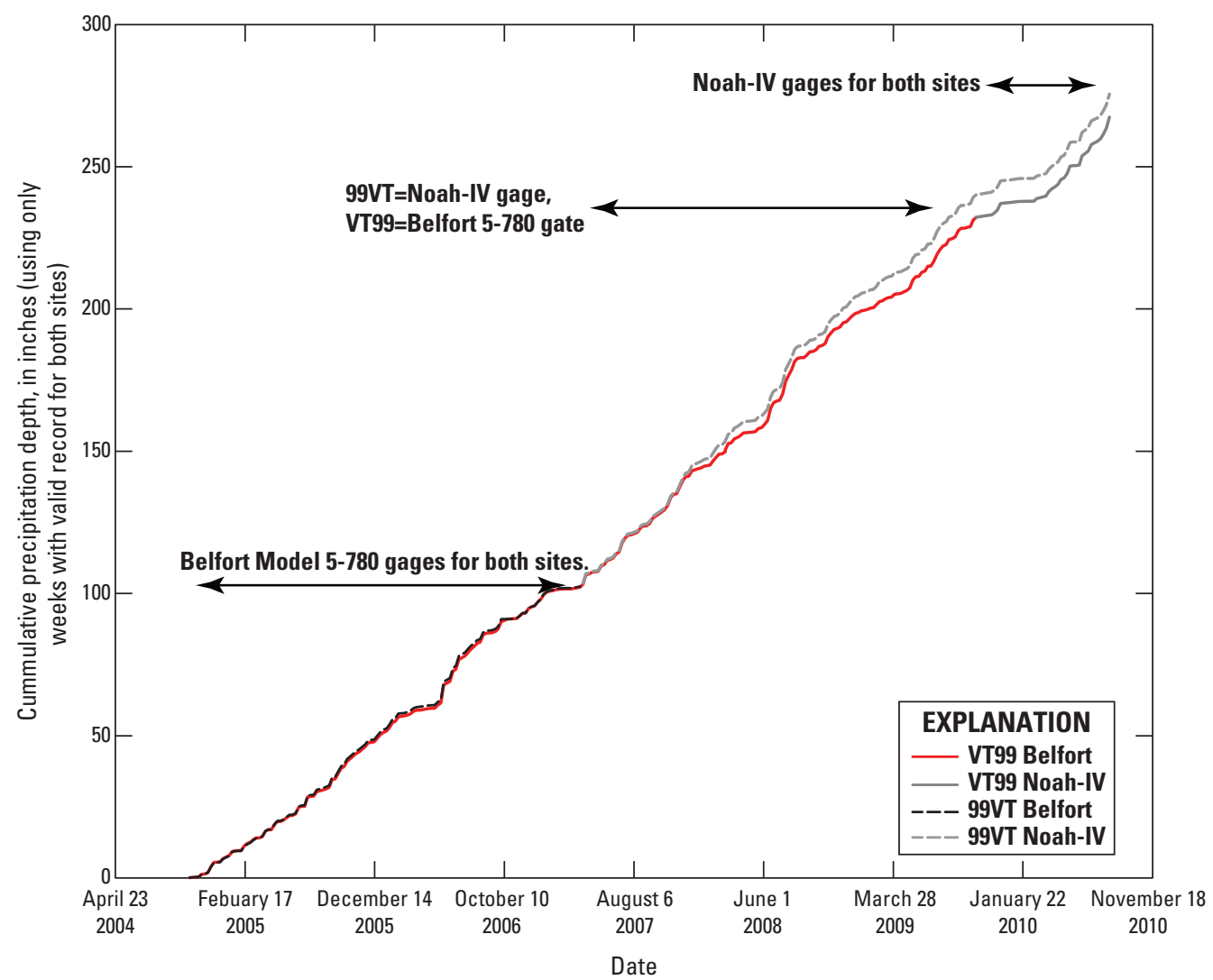

Figure 14. Cumulative weekly precipitation depth during water years 2005-10 for paired ETI Noah-IV precipitation gages at co-located National Trends Network sites VT99 and 99VT, Underhill, Vermont.

Because annual summaries of NTN data describe wetdeposition chemistry in terms of concentration and deposition (National Atmospheric Deposition Program, 2011), statistical summaries for both the concentration and deposition of constituents are provided. The weekly precipitation depth associated with each recording rain gage was used to calculate deposition values at the co-located sites by multiplying analyte concentration in $\mathrm{mg} / \mathrm{L}$ by 0.10 times the precipitation depth in centimeters $(\mathrm{cm})$ to yield deposition in kilograms per hectare $(\mathrm{kg} / \mathrm{ha})$.

\section{IL11/96IL and VT99/99VT}

Two N-CON collectors were co-located with existing ACM collectors at NADP/NTN sites VT99, Underhill Center, VT, and IL11, Bondville, Champaign, Ill. Site identifiers for the N-CON sites are 99VT and 96IL, which are co-located with ACM sites VT99 and IL11, respectively. The period of record (that is, the study period) for the IL11/96IL collectors is November 11, 2008-August 3, 2010. The period of record for the VT99/99VT collectors is October 7, 2008-August 3, 2010.

Co-located N-CON collectors were installed within 5 to 15 meters of the original ACM collectors such that the paired collectors had similar fetch and bucket orifice elevation as the existing ACM collectors. Photographs of the N-CON collectors are shown in figure 13. Both sets of co-located collectors were operated using NADP/NTN protocols (Dossett and Bowersox, 1999).

\section{Mechanical Performance Comparison}

Reliability and durability of the N-CON collectors is related to the quality and types of samples collected. A comparison of sample types collected by the co-located ACM and $\mathrm{N}-\mathrm{CON}$ collectors is shown in table 9. The combined number of missed and bulk/undefined samples suggests that the performance of the N-CON collector at VT99 was superior to that of the ACM. At IL11, the same comparison favors the ACM, partly because the $\mathrm{N}-\mathrm{CON}$ was taken out of service several times to repair and modify the lid mechanism to meet performance requirements. The collector lid was lengthened to cover the bucket more effectively, and the guide arm axle was lengthened to prevent the guide arms from interfering with the bucket handle. The N-CON was not redeployed to $96 \mathrm{IL}$ immediately after the upgrades were completed. Even with these down times, 66 percent of the samples collected by the N-CON at $96 \mathrm{IL}$ were valid per NADP criteria. Because the N-CON was removed from service for repair and upgrades during the study period, the performance evaluation of the N-CON at IL11 is biased in comparison to the evaluation at VT99/99VT. 

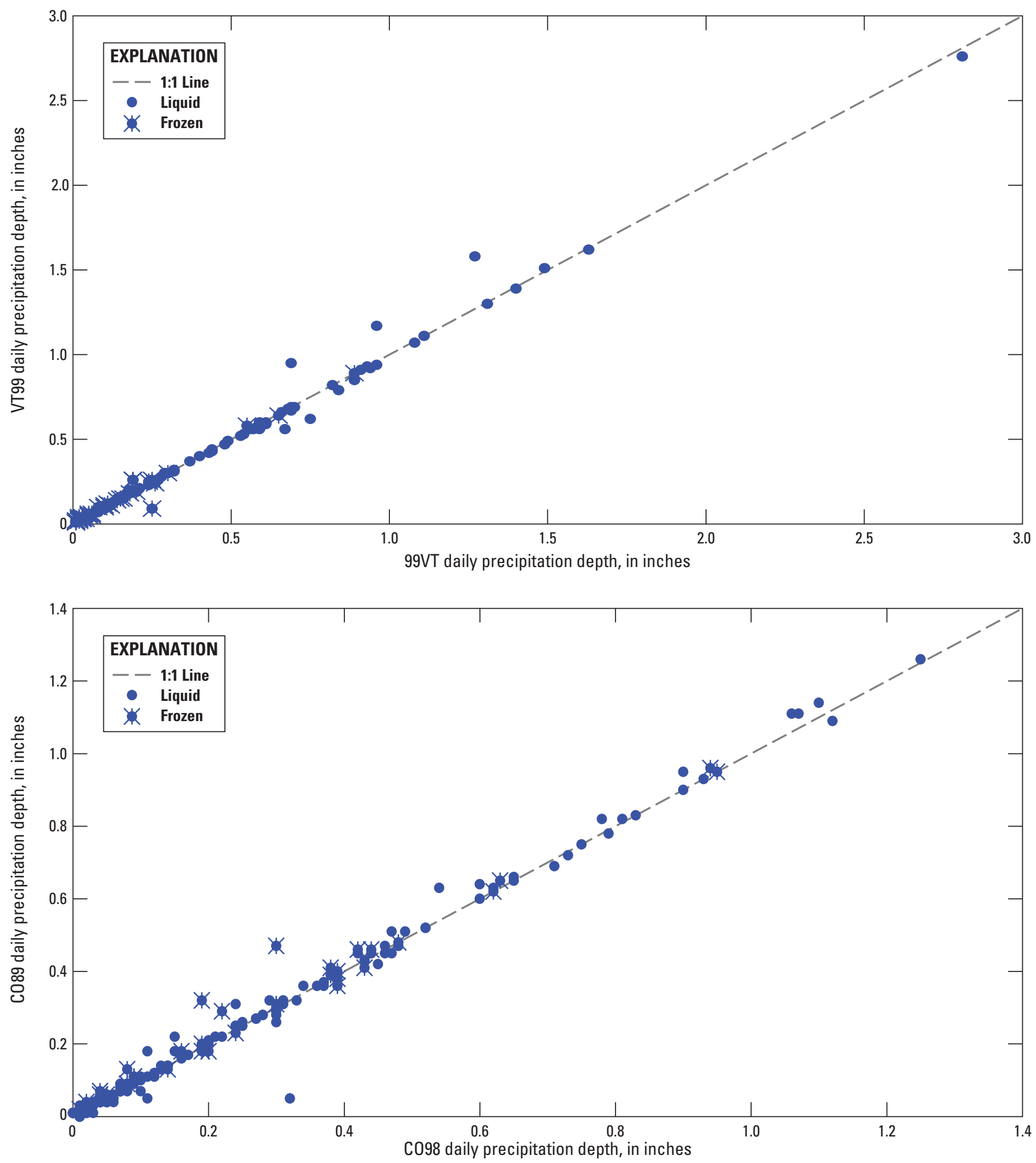

Figure 15. Daily precipitation depth measurements for paired ETI Noah-IV precipitation gages at co-located National Trends Network sites C098/C089, Loch Vale, Colorado and VT99/99VT, Underhill, Vermont, water year 2010. 
During the entire study period, the N-CON collector failed to operate correctly only once at 99VT; the cause of which is unclear. Following the failure, the collector performed as well as it had prior to the outage and required no service or replacement of the sensor or motor. By contrast, the ACM collector at VT99 operated in a continuously open configuration during both wet and dry periods (bulk mode) for many weeks during the winter due to motor failures.

\section{Precipitation Sensor Comparison}

The N-CON collector has an optical, Thies-brand infrared light sensor (Clima Precipitation Monitor model) to detect the onset and termination of precipitation events. This sensor was approved by NADP in 2009, and it has been used with N-CON MDN collectors since late 2006 as documented in the 2006 NADP Network Operations Subcommittee meeting minutes (http://nadp.sws.uiuc.edu/committees/minutes/ fall06/tc2006.pdf). The sensor triggers the collector motor to open the lid and expose the bucket to the atmosphere for sample collection, and then closes the lid when precipitation is no longer detected. Co-located collector lid openings (events) were recorded by the datalogger in the ETI Noah-IV electronic raingage at VT99/99VT. The dataloggers record the number of seconds within each 15-minute interval for the following events.

- Number of open-close collector-lid cycles,
- Wet exposure time: Time when the wet-side bucket is exposed to the atmosphere during periods when precipitation is measured by the rain gage,

- Dry exposure time: Time when the wet-side bucket is exposed to the atmosphere during periods when no precipitation is measured by the rain gage, and

- Missed exposure time: Time when the wet-side bucket lid is closed during periods when precipitation is measured by the rain gage.

At the IL11/96IL co-located sites, there was no open channel on the IL11 OTT Pluvio-N rain gage for recording 96IL collector lid events. Attempts to collect the 96IL collector-lid event data using another type of datalogger were unsuccessful.

Results in table 10 indicate that the N-CON had approximately 2 to 20 times more wet exposure time than the ACM and two to three times more dry exposure than the ACM. The $\mathrm{N}-\mathrm{CON}$ opens earlier than the ACM, and it typically stays open longer, especially during light precipitation events such as snow flurries. These data indicate that the N-CON's optical sensor is more sensitive than the ACM grid sensor in terms of opening and closing the collector.

Data in figure 17 indicate that the N-CON typically catches slightly more precipitation than the ACM because a majority of points in the scatter plot are located on the N-CON side of the 1-to-1 line. These results are consistent with those in table 10 as more wet exposure time is consistent with increased catch.

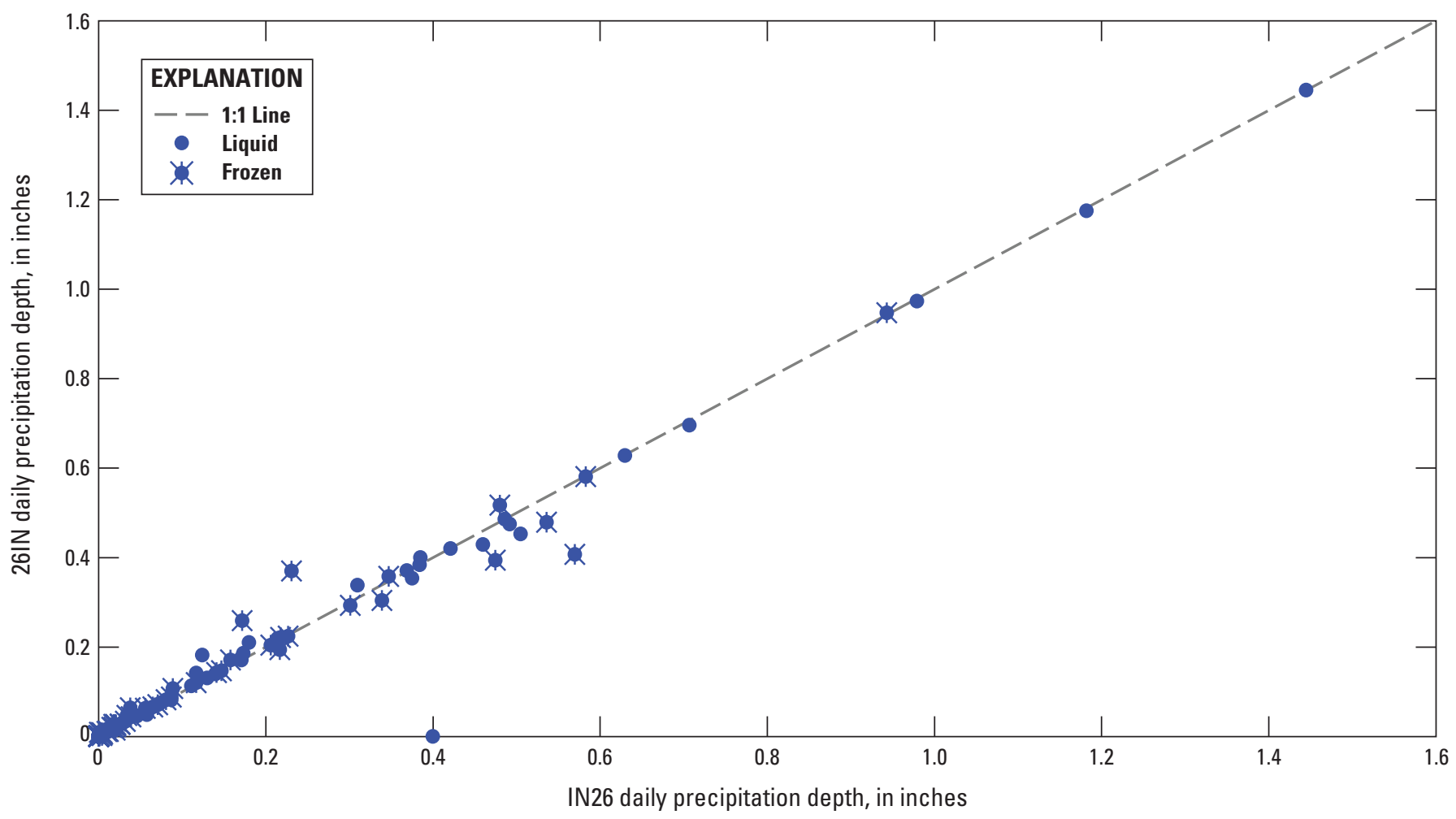

Figure 16. Daily precipitation depth measurements at co-located Mercury Deposition Network sites IN26 and 26IN, Harrison State Park, Indiana, water year 2010 [ACM, Aerochem Metrics; MDN, Mercury Deposition Network]. 
Table 9. Sample types for co-located AeroChem Metrics (ACM) and N-CON NTN (N-CON) collectors at sites VT99/99VT and IL11/96IL during water years 2009-2010.

[ACM, Aerochem Metrics collector; N-CON, N-CON Systems collector; W-coded, samples with sufficient volume for analysis without dilution; Missed, precipitation occurred but was not sampled; WD-coded: samples diluted to obtain sufficient volume for analysis; T-coded, trace amount samples not analyzed due to insufficient volume for complete analysis; D-coded, dry week; Bulk, collector open to atmosphere continuously during both wet and dry periods; Undefined, status of collector unknown; Valid samples, not bulk, undefined, contaminated, or missed.]

\begin{tabular}{|c|c|c|c|c|}
\hline \multirow[b]{4}{*}{ Sample Types } & \multicolumn{4}{|c|}{ Periods of record } \\
\hline & \multicolumn{2}{|c|}{ 10/07/08-08/10/10 } & \multicolumn{2}{|c|}{$11 / 11 / 08-08 / 10 / 10$} \\
\hline & \multicolumn{4}{|c|}{ Numbers of samples by site ID/collector type } \\
\hline & VT99/ACM & 99VT/N-CON & IL11/ACM & 96IL/N-CON \\
\hline $\begin{array}{l}\text { W-coded, \& } \\
\text { Non-contaminated }\end{array}$ & 26 & 46 & 55 & 45 \\
\hline W-coded, contaminated & 42 & 45 & 12 & 20 \\
\hline Missed & 17 & 1 & 0 & 2 \\
\hline $\begin{array}{l}\text { WD- \& T-coded, } \\
\text { Non-contaminated }\end{array}$ & 1 & 0 & 9 & 8 \\
\hline Bulk/Undefined & 3 & 1 & 1 & 5 \\
\hline D-Coded & 4 & 2 & 3 & 0 \\
\hline T-coded, contaminated & 3 & 1 & 2 & 2 \\
\hline Percent valid samples & 39 & 51 & 82 & 66 \\
\hline
\end{tabular}

Table 10. Comparison of event data for co-located ACM and N-CON NTN precipitation collectors at co-located sites VT99/99VT during water years 2009-10.

[ACM, Aerochem Metrics Model 301 precipitation collector; N-CON, $\mathrm{N}-\mathrm{CON}$ Systems, Inc. bucket-type precipitation collector]

\begin{tabular}{ccccc}
\hline & \multicolumn{3}{c}{ Water Year 20091 } & \multicolumn{2}{c}{ Water Year 2010' } \\
\cline { 2 - 5 } & \multicolumn{4}{c}{ Site/collector type } \\
\cline { 2 - 5 } Event data & $\begin{array}{r}\text { VT99 } \\
\text { ACM }\end{array}$ & $\begin{array}{c}\text { 99VT } \\
\text { N-CON }\end{array}$ & $\begin{array}{c}\text { VT99 } \\
\text { ACM }\end{array}$ & $\begin{array}{c}\text { 99VT } \\
\text { N-CON }\end{array}$ \\
\hline $\begin{array}{c}\text { Collector lid cycles } \\
\text { (open and close) }\end{array}$ & 1,425 & 6,934 & 1,273 & 8,072 \\
$\begin{array}{c}\text { Wet exposure time } \\
\text { (hours) }\end{array}$ & 50 & 1,064 & 498 & 879 \\
$\begin{array}{c}\text { Dry exposure time } \\
\text { (hours) }\end{array}$ & 30.3 & 107 & 45.5 & 94 \\
\hline
\end{tabular}

${ }^{1} 12$-month period beginning October 1 and ending September 30 of the designated year.

\section{Sample Chemistry Comparison}

\section{Weekly Values}

To limit potential variability in the chemical data from uncontrolled conditions, sample chemistry concentrations from the co-located ACM and N-CON collectors were compared only for paired, weekly, W-coded samples; this yielded 131 sample pairs out of the $291 \mathrm{~W}$-coded samples from the four collectors at the Vermont and Illinois sites (table 9). Of the $131 \mathrm{~W}$-coded sample pairs, 65 sample pairs had no visible contamination. The non-contaminated subset was analyzed separately because contamination can increase variability in co-located sampler results (Gordon, 1999). All other sample types were excluded from analysis.

Precipitation at the beginning of an event often has higher constituent concentrations due to washout of materials from the atmosphere. Subsequent precipitation becomes more dilute as events progress (Aikawa and others, 2009; Schroder and others, 1986), but not always (Lynch and others, 1989; Colin and others, 1987). Therefore, the effect of the collector lid opening early or late can be important to comparison of sample chemistry results obtained for each collector.

Paired weekly ACM-minus-N-CON concentration differences were evaluated for bias. Median ACM-minus-N-CON weekly concentration differences are all negatively signed except for hydrogen-ion concentration, which indicates that $\mathrm{N}-\mathrm{CON}$ concentrations generally are higher than ACM concentrations (table 11). Median weekly concentration differences 
Table 11. Median weekly ACM-minus-N-CON concentration differences and results of sign test for bias between Aerochem Metrics and N-CON precipitation collectors.

[Shaded values are significant at $\alpha=0.10 ; \mathrm{mg} / \mathrm{L}$, milligrams per liter; $\mu \mathrm{Eq} / \mathrm{L}$, micro-equivalents per liter; $\mathrm{mS} / \mathrm{cm}$, micro-Siemens per centimeter; $\mathrm{mL}$, milliliters]

\begin{tabular}{|c|c|c|c|}
\hline $\begin{array}{c}\text { Sample type } \\
\text { Censoring }^{1}\end{array}$ & Analyte & $\begin{array}{c}\text { Median weekly } \\
\text { concentration differences } \\
\text { (mg/L unless otherwise shown) }\end{array}$ & $\begin{array}{c}\text { Sign test } \\
p \text {-value }\end{array}$ \\
\hline \multirow{11}{*}{$\begin{array}{l}\text { All W-Coded } \\
\quad \mathrm{N}=131\end{array}$} & Calcium & -0.017 & $<0.0001$ \\
\hline & Magnesium & -0.003 & $<0.0001$ \\
\hline & Sodium & -0.003 & $<0.0001$ \\
\hline & Potassium & -0.003 & $<0.0001$ \\
\hline & Ammonium & -0.050 & $<0.0001$ \\
\hline & Chloride & -0.007 & $<0.0001$ \\
\hline & Nitrate & -0.103 & $<0.0001$ \\
\hline & Sulfate & -0.090 & $<0.0001$ \\
\hline & Hydrogen-Ion $(\mu \mathrm{Eq} / \mathrm{L})$ & 0.180 & 0.2831 \\
\hline & Specific Conductance $(\mathrm{mS} / \mathrm{cm})$ & -0.640 & $<0.0001$ \\
\hline & Sample Volume (mL) & -43.4 & $<0.0001$ \\
\hline \multirow{11}{*}{$\begin{array}{l}\text { Non-Contaminated } \\
\text { W-Coded Only } \\
\mathrm{N}=65\end{array}$} & Calcium & -0.019 & $<0.0001$ \\
\hline & Magnesium & -0.003 & $<0.0001$ \\
\hline & Sodium & -0.004 & $<0.0001$ \\
\hline & Potassium & -0.003 & $<0.0001$ \\
\hline & Ammonium & -0.060 & $<0.0001$ \\
\hline & Chloride & -0.008 & $<0.0001$ \\
\hline & Nitrate & -0.110 & $<0.0001$ \\
\hline & Sulfate & -0.093 & $<0.0001$ \\
\hline & Hydrogen-Ion $(\mu \mathrm{Eq} / \mathrm{L})$ & 0.181 & 0.7035 \\
\hline & Specific Conductance (mS/cm) & -0.710 & $<0.0001$ \\
\hline & Sample Volume $(\mathrm{mL})$ & -37.0 & 0.0026 \\
\hline
\end{tabular}

${ }^{1} \mathrm{All} \mathrm{W}$-coded samples and non-contaminated W-coded samples analyzed separately.

${ }^{2} p$-value: probability of incorrectly rejecting the null hypothtesis, $H o$ : The median weekly ACM-minus-N-CON differences are not significantly different from zero, which is rejecting that no bias exists between collectors with respect to concentration.

between the ACM and N-CON were between 0.003 to $0.008 \mathrm{mg} / \mathrm{L}$ for magnesium, sodium, potassium, and chloride, and between 0.017 to $0.110 \mathrm{mg} / \mathrm{L}$ for calcium, ammonium, nitrate, and sulfate. This could be due to the N-CON collector opening earlier than the ACM at the onset of precipitation, thereby catching more washout at the beginning of precipitation events (Lynch and others, 1989). In addition, dry deposition of aerosols into the sample bucket is likely greater for the $\mathrm{N}-\mathrm{CON}$ because dry exposure time is much greater for the $\mathrm{N}-\mathrm{CON}$ than the ACM (table 10). Calcium- and magnesiumcontaining particles are commonly components of washout. When dissolved in the sample, these particles can buffer hydrogen ion concentrations and thus account for lower ACM hydrogen-ion concentrations. Sign test results for significant differences in the central location of all paired, W-coded samples and W-coded, non-contaminated samples indicate that concentrations of calcium, magnesium, sodium, potassium, ammonium, chloride, nitrate, and sulfate, and specific conductance and sample volume were significantly different between the two collectors at the $(\alpha=0.10)$ significance level $(90$ percent confidence level) regardless of whether the contaminated samples were censored or remained in the test data set (table 11). Hydrogen-ion concentrations were not significantly different between the two collectors at the $\alpha=0.10$ significance level.

The N-CON generally catches larger sample volumes than the ACM, and N-CON samples have higher specific conductance. The distributions of the concentrations, specific conductance, and sample volume differences are illustrated in figures 18 and 19. Boxplots in figures 18 and 19 indicate that the median ACM-minus-N-CON differences are nearly 

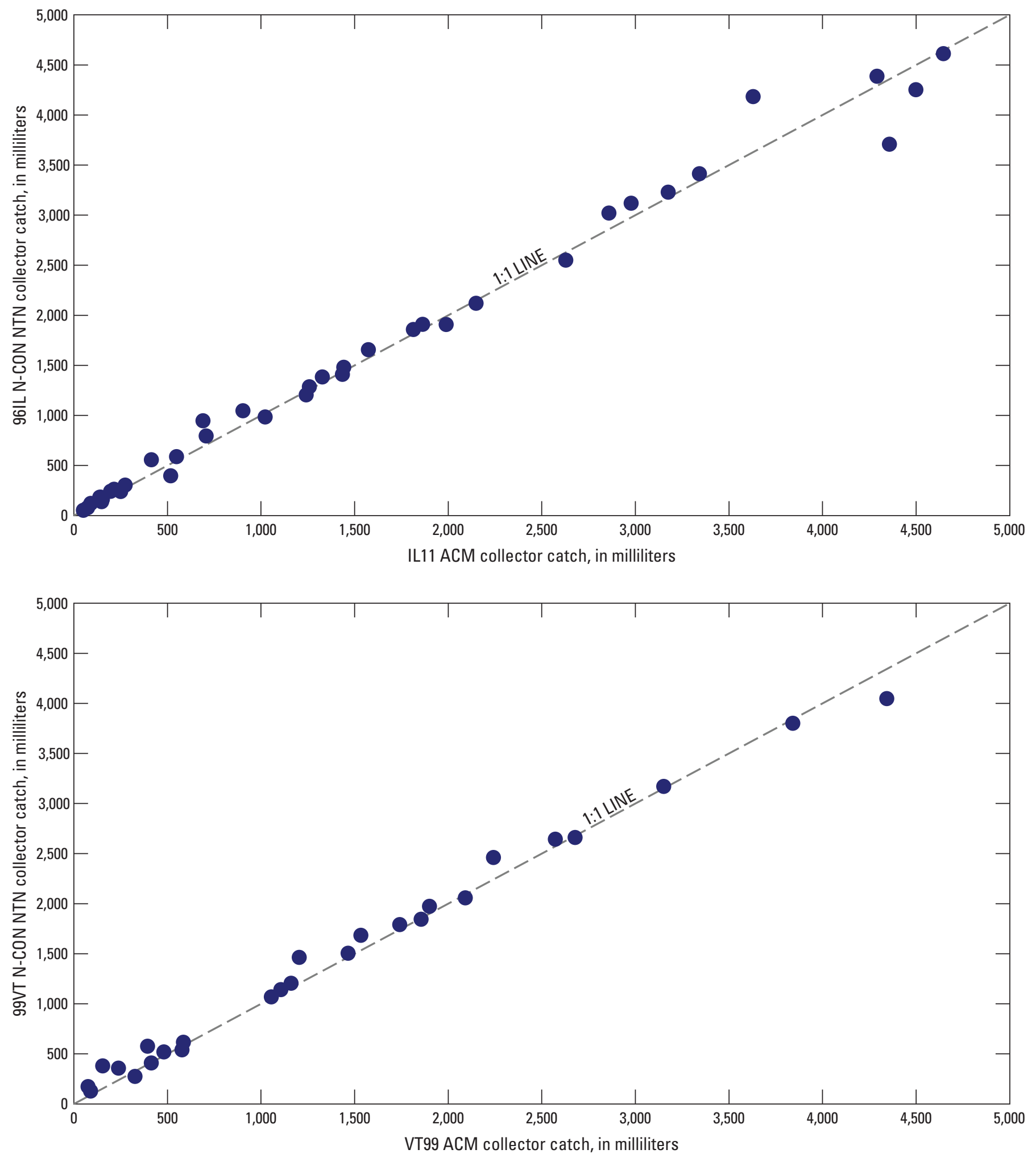

Figure 17. AeroChem Metrics (ACM) and N-CON NTN (N-CON) precipitation catch at co-located sites IL11/96IL and VT99/99VT during 2008-10. 


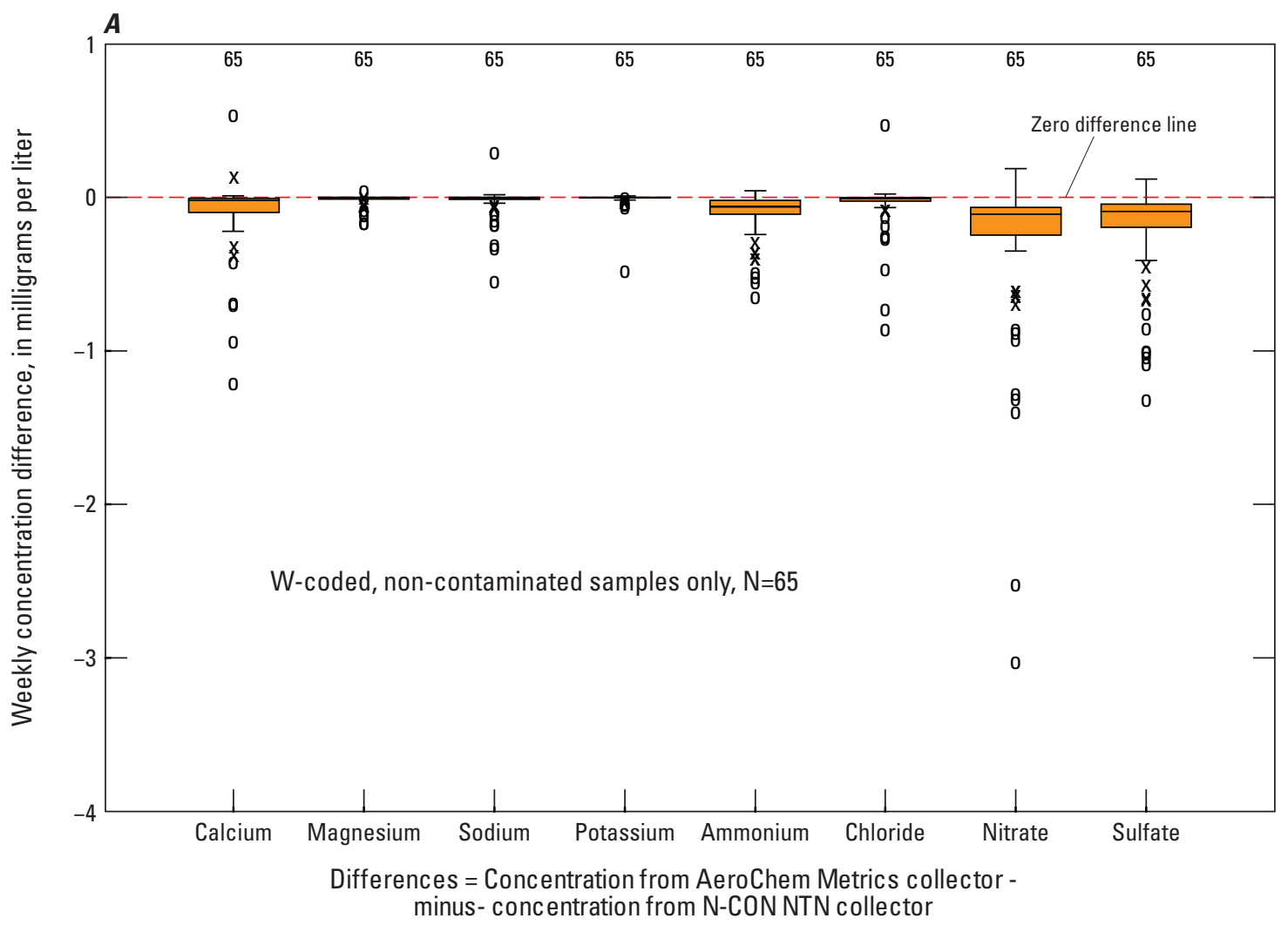

EXPLANATION

65 Number of values

- Upper detached

$x$ Upper outside

Tpper adjacent

75th percentile

Median

25th percentile

Lower adjacent

$x$ Lower outside

o Lower detached

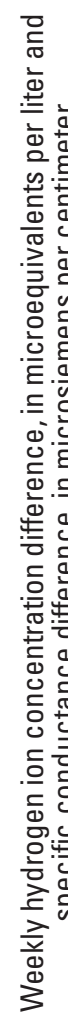

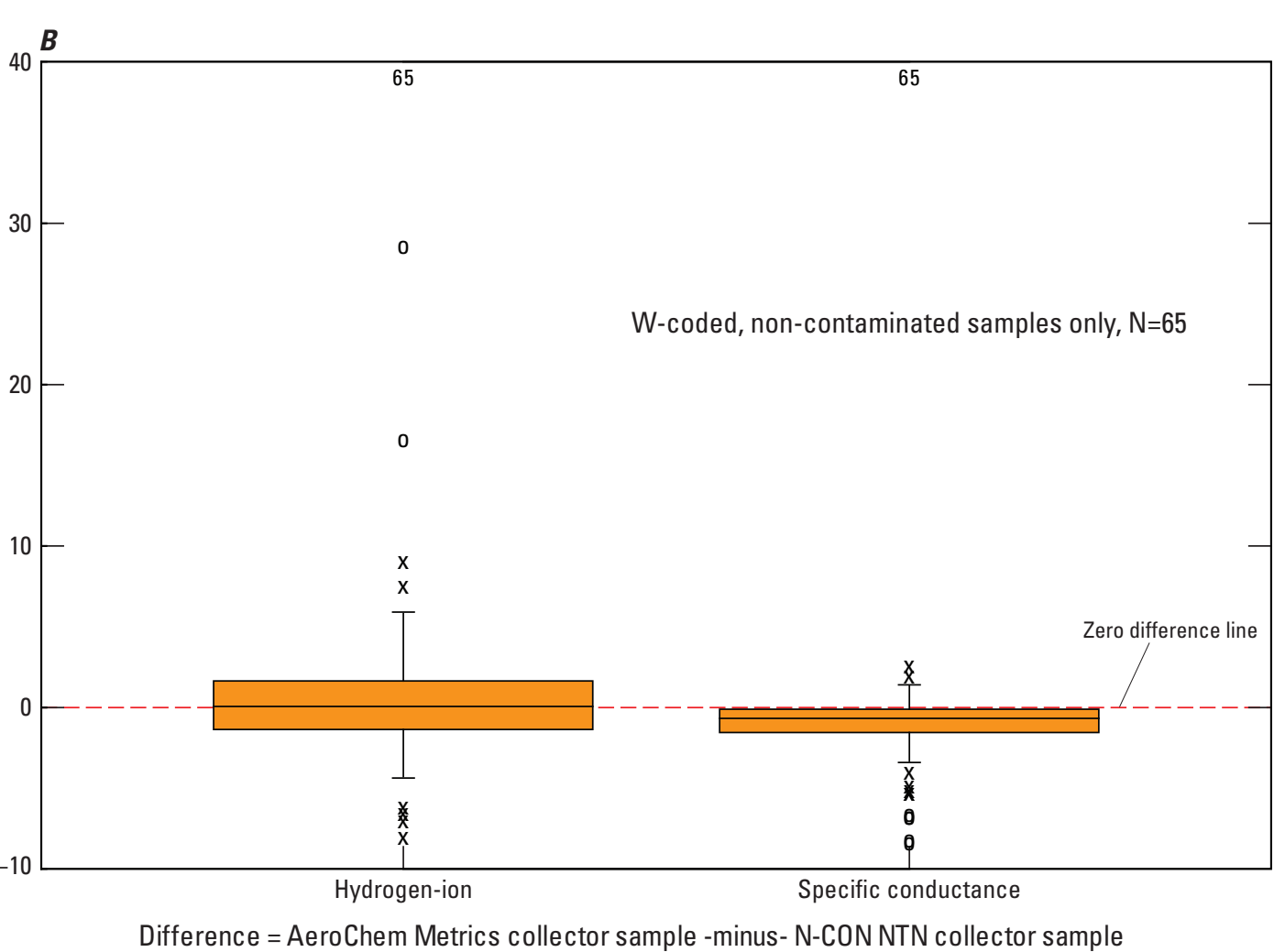

Figure 18. Aerochem Metrics-minus-N-CON weekly $A$, concentration and $B$, hydrogen-ion and specific-conductance differences for paired samples from co-located sites IL11/96IL and VT99/99VT during water years 2009-10. 


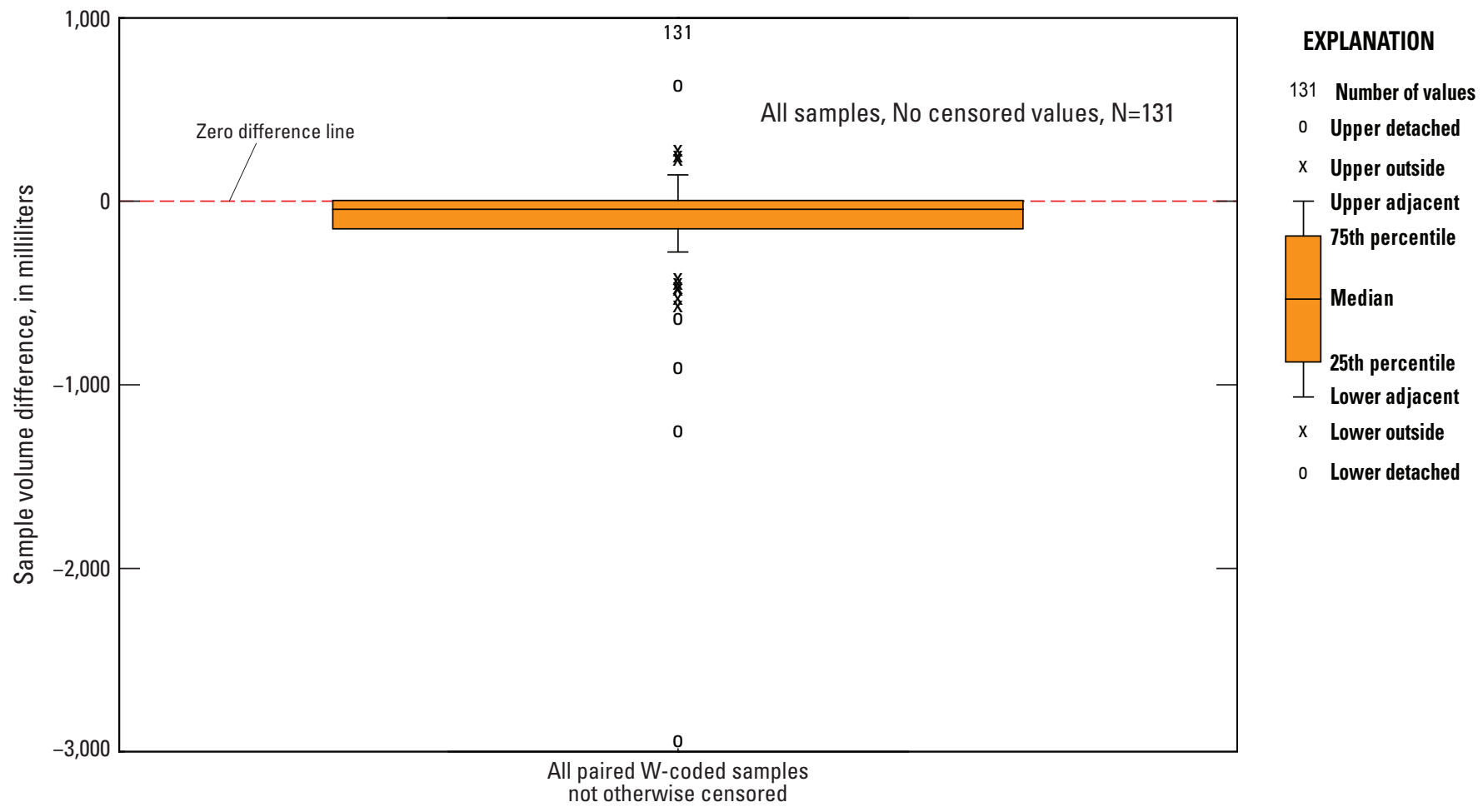

Difference $=$ AeroChem Metrics sample volume -minus- N-CON NTN sample volume

Figure 19. Aerochem Metrics-minus-N-CON weekly sample-volume differences for paired samples from co-located sites IL11/96IL and VT99/99VT during water years 2009-10.

Table 12. Annual precipitation-weighted mean concentration and deposition differences for co-located AeroChem Metrics and N-CON collectors at NADP sites VT99/99VT and IL11/96IL for weeks where data are available for both collectors during water year 2009.

[N=131 W-coded, co-located samples; except for Hydrogen Ion, where $\mathrm{N}=130 ; \mathrm{mg} / \mathrm{L}$, miiligrams per liter; $\mathrm{kg} / \mathrm{ha}$, kilograms per hectare; $\mathrm{N}-\mathrm{CON}$, N-CON Systems, Inc. bucket-type precipitation collector; Aerochem, Aerochem Metrics model 301 precipitation collector]

\begin{tabular}{|c|c|c|c|c|c|c|}
\hline \multirow[t]{2}{*}{ Analyte } & \multicolumn{2}{|c|}{$\begin{array}{l}\text { Annual precipitation- } \\
\text { weighted mean } \\
\text { concentration differences }{ }^{1} \\
\text { (mg/L) }\end{array}$} & \multicolumn{2}{|c|}{$\begin{array}{l}\text { Annual deposition } \\
\text { differences } \\
\text { (kg/ha) }\end{array}$} & \multicolumn{2}{|c|}{$\begin{array}{c}2009 \\
\text { Annual precipitation-weighted } \\
\text { mean concentration and } \\
\text { deposition percent differences }\end{array}$} \\
\hline & VT99/99VT & IL11/96IL & VT99/99VT & IL11/96IL & VT99/99VT & IL11/96IL \\
\hline Calcium & -0.017 & -0.033 & -0.19 & -0.43 & -30 & -16 \\
\hline Magnesium & -0.003 & -0.007 & -0.03 & -0.09 & -37 & -24 \\
\hline Sodium & -0.006 & -0.010 & -0.06 & -0.13 & -29 & -18 \\
\hline Potassium & -0.012 & -0.020 & -0.13 & -0.26 & -74 & -72 \\
\hline Ammonium & -0.030 & -0.023 & -0.32 & -0.30 & -14 & -5 \\
\hline Chloride & -0.012 & -0.013 & -0.13 & -0.17 & -28 & -13 \\
\hline Nitrate & -0.085 & -0.105 & -0.92 & -1.34 & -12 & -11 \\
\hline Sulfate & -0.071 & -0.095 & -0.77 & -1.21 & -10 & -7 \\
\hline Hydrogen Ion & -0.0004 & 0.0011 & -0.004 & 0.014 & -3 & 8 \\
\hline
\end{tabular}

${ }^{1}$ Difference $=$ Aerochem concentration minus N-CON concentration.

${ }^{2}$ Difference $=$ Aerochem deposition minus N-CON deposition.

${ }^{3}$ Percent difference $=100 \times[($ Aerochem value $-\mathrm{N}-\mathrm{CON}$ value $) /($ Aerochem value $)]$. 
zero for all chemical parameters, but the differences are predominantly negative, indicating that values for the $\mathrm{N}-\mathrm{CON}$ collector are higher than for the ACM collector.

\section{Annual Values}

Table 12 shows a comparison of annual precipitationweighted mean concentration and total deposition value differences. The annual values indicate that precipitation-weighted mean concentrations were higher for $\mathrm{N}-\mathrm{CON}$ collectors than for ACM collectors except for hydrogen ion at IL11/96IL. Annual precipitation-weighted mean concentration and deposition percent differences relative to the ACM collector range between -14 percent (ammonium, nitrate, and sulfate) and 8 percent (hydrogen ion); percent differences for calcium, magnesium, sodium, potassium, and chloride range from -74 percent to -13 percent.

\section{Precipitation-Type Bias}

A Wilcoxon-Signed Ranks test (table 13), which compared the central locations of two groups: (1) samples which were at least partly composed of frozen precipitation and (2) samples composed of no frozen precipitation, indicated

Table 13. Results of Wilcoxon Signed Ranks test for precipitation-type bias in wet-deposition solute concentrations, specific conductance, and sample volume with respect to precipitation collector type and presence of frozen versus liquid precipitation.

[N Frozen samples/N Liquid samples: numbers of samples containing frozen precipitation versus liquid only; Shading indicates rejection of $H o$ with 95 -percent confidence; $\mathrm{mg} / \mathrm{L}$, milligrams per liter; $\mu \mathrm{Eq} / \mathrm{L}$, micro-equivalents per liter; $\mathrm{mS} / \mathrm{cm}$, micro-Siemens per centimeter; $\mathrm{mL}$, milliliters]

\begin{tabular}{|c|c|c|c|c|}
\hline $\begin{array}{l}\text { Sample type } \\
\text { censoring }\end{array}$ & Analyte & $\begin{array}{c}\mathbf{N} \\
\text { Frozen samples }\end{array}$ & $\begin{array}{c}\mathbf{N} \\
\text { Liquid samples }\end{array}$ & $\begin{array}{c}\text { Wilcoxon signed ranks } \\
p \text {-value }\end{array}$ \\
\hline \multirow{11}{*}{$\begin{array}{l}\text { All W-Coded } \\
\text { Samples } \\
\text { Combined } \\
\mathrm{N}=131^{1}\end{array}$} & Calcium & 33 & 87 & $<0.0001$ \\
\hline & Magnesium & 33 & 87 & $<0.0001$ \\
\hline & Sodium & 33 & 87 & $<0.0001$ \\
\hline & Potassium & 33 & 87 & 0.0969 \\
\hline & Ammonium & 33 & 86 & 0.1455 \\
\hline & Chloride & 33 & 87 & $<0.0001$ \\
\hline & Nitrate & 33 & 87 & $<0.0001$ \\
\hline & Sulfate & 33 & 87 & $<0.0001$ \\
\hline & Hydrogen-Ion $(\mu \mathrm{Eq} / \mathrm{L})$ & 33 & 86 & 0.7896 \\
\hline & Specific Conductance (mS/cm) & 33 & 86 & 0.0003 \\
\hline & Sample Volume $(\mathrm{mL})$ & 33 & 87 & $<0.0001$ \\
\hline \multirow{11}{*}{$\begin{array}{l}\text { Non-Contaminated } \\
\text { W-Coded Only } \\
\text { Samples } \\
\text { Combined } \\
\mathrm{N}=65^{1}\end{array}$} & Calcium & 16 & 41 & 0.0007 \\
\hline & Magnesium & 16 & 41 & 0.0007 \\
\hline & Sodium & 16 & 41 & 0.0079 \\
\hline & Potassium & 16 & 41 & 0.1388 \\
\hline & Ammonium & 16 & 40 & 0.3497 \\
\hline & Chloride & 16 & 41 & 0.0118 \\
\hline & Nitrate & 16 & 41 & 0.0163 \\
\hline & Sulfate & 16 & 41 & 0.0170 \\
\hline & Hydrogen-Ion $(\mu \mathrm{Eq} / \mathrm{L})$ & 16 & 40 & 0.7935 \\
\hline & Specific Conductance (mS/cm) & 16 & 40 & 0.0009 \\
\hline & Sample Volume (mL) & 16 & 41 & 0.0415 \\
\hline
\end{tabular}

${ }^{1} \mathrm{~W}$-coded samples, samples with sufficient volume for analysis without dilution; Not all samples are represented by all analytes.

${ }^{2} p$-value: probability of incorrectly rejecting the null hypothtesis, $H o$ : There is no bias between the collectors with respect to absolute percent differences due to frozen precipitation. 
a significant $(\alpha=0.05)$ precipitation-type bias between the collectors for all parameters evaluated except for potassium, ammonium, and hydrogen-ion concentrations. At least twice as many pairs of liquid-only precipitation samples were collected by both collectors compared to pairs of samples containing frozen precipitation. This difference in the number of samples could affect the outcome of the hypothesis test. The N-CON was observed to be open for long periods while the ACM remained closed during light snow events (Mim Pendelton, VT99/99VT site operator, Proctor Maple Research Center, verbal commun., April 2009; table 13).

Catch efficiency is represented by the ratio of precipitation depth in the collector bucket to the precipitation depth in the rain gage. A catch efficiency of 1.0 indicates ideal collection of the entire amount of precipitation that was recorded by the rain gage during the week. Catch efficiencies less than 1.0 indicate incomplete collection of the weekly precipitation recorded by the rain gage, and catch efficiencies greater than 1.0 indicate problems with the rain gage or collection of precipitation blown or bounced into the bucket from nearby surfaces or other unusual conditions. Time series plots of ACM-minus-N-CON concentration differences shown in figure 20 indicate consistent patterns of decreased catch efficiency during winter months, but generally less so for the $\mathrm{N}-\mathrm{CON}$ than for the ACM.

The results suggest that retrofit of existing ACM collectors with new N-CON collectors would result in less than a -10 percent shift in annual precipitation-weighted mean concentrations and total annual deposition for hydrogen-ion, and a small positive shift (less than +15 percent) for ammonium, nitrate, and sulfate concentration. This has implications for selection of trend-analysis techniques for long-term trends that include changes in field instrumentation. These data, however, are indicative of specific conditions experienced by the co-located equipment. More data are needed from additional co-located sampling to further evaluate precipitation-type bias of N-CON collectors. The degree and sign of bias could be site specific and depend on the proportion of precipitation that occurred as light versus heavier frozen precipitation versus liquid precipitation.

\section{Mercury Deposition Network Quality Assurance Programs}

The USGS operated three QA programs for MDN during 2009-10: (1) a system-blank program, (2) an interlaboratory-comparison program, and (3) a blind-audit program. A brief history of these programs is provided by Wetherbee and others (2010). The MDN system-blank program is similar to the NTN field-audit program, whereby the effects of onsite, environmental exposure, handling, and shipping of samples are evaluated. The MDN interlaboratory-comparison program quantifies variability and bias of MDN analytical data provided by the Mercury (Hg) Analytical Laboratory (HAL), which is Frontier Global Sciences, Inc., formerly located in Seattle, Washington (Wash.), and now relocated to Bothell, Wash., in 2012. Potential bias in HAL sample analyses for total mercury concentrations was evaluated further by a blind-audit program. Protocols for the USGS external QA programs for MDN are described in detail by Latysh and Wetherbee (2007).

\section{System-Blank Program}

Each quarter during 2009-10, approximately 26 MDN site operators received a system-blank sample from USGS for processing and submission to HAL. After a week without wet deposition, site operators poured one-half of the volume of their system-blank solution through the sample train into the sample bottle. The solution that washed through the sample train is called the system-blank sample, and the solution remaining in the original sample bottle is called the bottle sample. Both system-blank and bottle samples were sent together to HAL for total $\mathrm{Hg}$ analysis. HAL provided the system-blank data to USGS, and system-sample minus bottlesample differences were calculated by USGS. The systemblank program is described by Wetherbee and others (2010).

Of 197 system-blank samples shipped to MDN sites during 2009-10, 114 (58 percent) responses were received during 2009-10. Of the system-blank samples received by the HAL during 2009-10, 15 sites reported that they did not have a dry week during their 6- to 12-month submission period, and an additional 15 sites reported problems with the sample (such as a cracked bottle or leaking sample). Responses for an additional 17 samples shipped in 2008 were processed in 2009 (included herein), and responses for 20 samples shipped in 2010 were processed in 2011(not included herein). System blanks submitted without a corresponding bottle sample were eliminated; this resulted in 104 paired system and bottle samples analyzed. In addition, 27 unopened bottle samples were returned to the HAL and analyzed, which were considered to be trip blanks.

\section{Network Maximum Contamination Levels for Mercury}

The 90-percent upper confidence limits (UCLs) were calculated for each percentile between the 5th and 99th percentiles of the system-sample minus bottle-sample differences using the binomial probability distribution function in SAS (SAS Institute, Inc., 2001). The UCLs for selected percentiles of these differences for 2009-10, are shown in figure 21. The 90-percent UCL for the 90th percentile of the concentration differences is interpreted as the maximum contamination in MDN samples with statistical confidence. For the 2009-10 study period, the maximum $\mathrm{Hg}$ contamination level was 1.545 nanograms per liter (ng/L). In other words, the maximum contamination in MDN samples during 2009-10 was not greater than $1.545 \mathrm{ng} / \mathrm{L}$ with 90 -percent confidence, and also, no more than 10 percent of the MDN samples had 


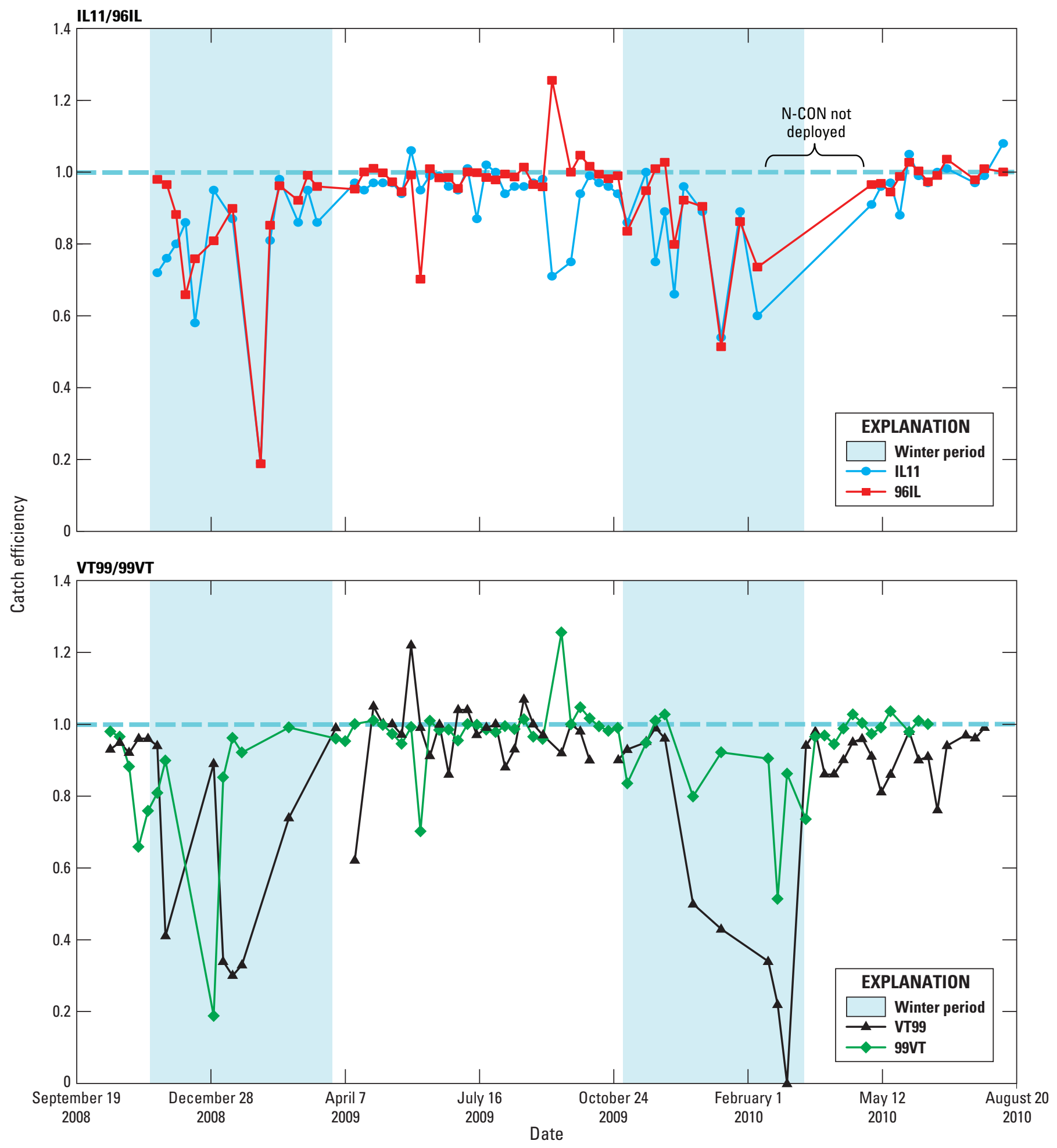

Figure 20. Weekly catch efficiency for co-located Aerochem Metrics (ACM) and N-CON precipitation collectors at IL11/96IL and VT99/99VT, water years 2009-10. 


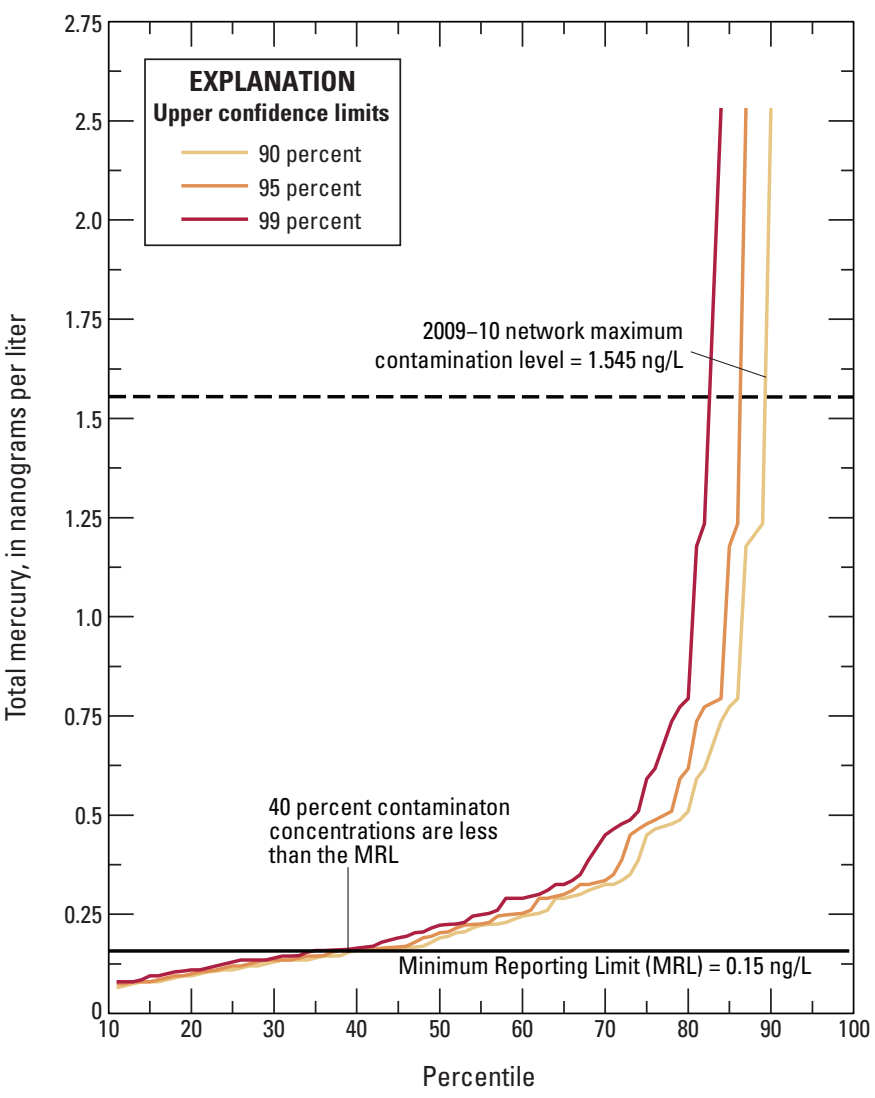

Figure 21. Upper confidence limits on mercury contamination concentration percentiles determined by 2009-10 system blank samples. (ng/L, nanograms per liter)

Table 14. Three-year moving average 90-percent upper confidence limits reported as network maximum contamination levels for total mercury $(\mathrm{Hg})$ concentration and mass estimated by system-blank samples, 2004-10.

[\%, percent; UCL, upper confidence limit; Hg, mercury; ng, nanograms; ng $\mathrm{Hg} / \mathrm{L}$, nanograms mercury per liter]

\begin{tabular}{ccc}
\hline \multirow{2}{*}{$\begin{array}{c}\text { 3-year } \\
\text { period }\end{array}$} & \multicolumn{2}{c}{ Network maximum contamination levels } \\
\cline { 2 - 3 } & $\begin{array}{c}\text { Concentration } \\
\text { (ng Hg/L) }\end{array}$ & $\begin{array}{c}\text { Mass } \\
\text { (ng Hg per } \\
\text { sample) }\end{array}$ \\
\hline $2004-06$ & 0.412 & 0.095 \\
$2005-07$ & 1.067 & 0.136 \\
$2006-08$ & 2.170 & 0.233 \\
$2007-09$ & 3.476 & 0.325 \\
$2008-10$ & 4.260 & 0.325 \\
\hline
\end{tabular}

190-percent UCL on 90th percentile of system-blank Hg contamination calculated on a concentration or mass basis. contamination concentrations exceeding $1.545 \mathrm{ng} / \mathrm{L}$ with 90 percent confidence. The 1.545 contamination concentration is approximately equal to the second percentile of all MDN field data, which is $1.620 \mathrm{ng} / \mathrm{L}$ (Mark Rhodes, NADP Program Office, written commun. July 12, 2012). Approximately 40 percent of the $\mathrm{Hg}$ contamination concentrations were less than the MRL (0.15 ng/L) during 2009-10; compared to over 85 percent in 2004 (Wetherbee and others, 2006).

The network maximum contamination level (NMCL) for total $\mathrm{Hg}$, as defined by this project, is the 3 -yr moving 90-percent UCL for the 90th percentile of the system-blank concentration differences. The MDN NMCL values dating back to the beginning of the program (2004) are shown in table 14. Values in table 14 have been updated from those previously by Wetherbee and others $(2009,2010)$, which were calculated with different time intervals rather than using a 3-yr moving average window. The NMCLs indicate that contamination in MDN samples steadily increased during 2004-10 when calculated on a concentration basis.

Evaluation of system-blank results collected to date revealed that NMCL's can be biased by sample volume. System-blank samples are shipped randomly to MDN sites in volumes of $125 \mathrm{~mL}, 500 \mathrm{~mL}$, and $1,000 \mathrm{~mL}$ to represent the middle 67 percent of MDN field sample volumes. When fewer $1,000 \mathrm{~mL}$-volume samples are processed and returned than $500 \mathrm{~mL}$ - or $125 \mathrm{~mL}$-volume samples, the resulting data set can become biased toward lower-volume samples. Therefore, it was determined that calculated NMCLs for Hg mass inherently have lower bias than NMCLs for $\mathrm{Hg}$ concentration.

Mass of $\mathrm{Hg}$ contamination was estimated for each sample. Because approximately half of the system-blank sample is poured into the collector, one-half of the total system-blank sample volume was multiplied by both the system-sample and bottle-sample concentrations to estimate the total $\mathrm{Hg}$ mass in each of the paired samples. Next, the UCLs of the percentiles of the system-minus-bottle sample Hg mass differences were calculated on a 3-yr moving basis. The results indicate that the 3-yr moving 90-percent UCLs on the 90th percentiles of $\mathrm{Hg}$ contamination mass (NMCL for Hg mass) in MDN samples has increased from 0.095 to $0.325 \mathrm{ng}$ per sample over 2004-10. Since 2006, there has been negligible change in the NMCLs for Hg mass (table 14).

Sources of the $\mathrm{Hg}$ contamination in MDN samples are likely in the field, not the laboratory. MDN interlaboratorycomparison program results for blanks, which are presented later in this report, indicate that laboratory $\mathrm{Hg}$ contamination is not problematic for the HAL. On the other hand, Wetherbee and others (2013) show how sample evaporation and associated $\mathrm{Hg}$ loss from MDN samples can occur in the field, especially for the modified-ACM MDN collectors. When evaporated sample condenses on the collector lid pad and the lid pad is not cleaned, then cross contamination between samples can occur. 


\section{MDN Interlaboratory-Comparison Program}

The objective of the MDN interlaboratory-comparison program is to estimate the variability and bias of HAL analytical data and to qualify these data for comparison with data from various monitoring networks, not accounting for the different onsite protocols used by different monitoring networks. Ten laboratories participated in the program during the study period: (1) ACZ Laboratories (ACZ), in Steamboat Springs, Colo.; (2) Atlantic Laboratory of Environmental Testing (ALET), in Moncton, New Brunswick, Canada; (3) Flett Research, Ltd., Winnipeg, Manitoba, Canada; (4) Frontier GeoSciences, Inc. (HAL), in Seattle, Wash.; (5) IVL-Swedish Environmental Institute (IVL), in Goteborg, Sweden; (6) Quebec Laboratory of Environmental Testing (LEEQ), in Montreal, Canada; (7) Northern Lake Service, Inc. (NLS), in Crandon, Wisconsin (Wisc.); (8) North Shore Analytical, Inc. (NSA), in Duluth, Minnesota; (9) Flemish Institute for Technological Research (VITO), in Mol, Belgium; and (10) USGS Wisconsin Mercury Laboratory (WML), in Middleton, Wisc.. ALET discontinued participation and LEEQ took its place in the program in April 2010. All laboratories analyze for low-level $\mathrm{Hg}$ in water using atomic fluorescence spectrometry methods similar to U.S. Environmental Protection Agency (USEPA) Method 1631 (U.S. Environmental Protection Agency, 2002).

During 2009-10, each participating laboratory received two samples per month consisting of 1-percent hydrochloric

Table 15. Most probable values for solutions used during 2009-10 for the U.S. Geological Survey Mercury Deposition Network interlaboratory-comparison program.

[Hg, mercury; MPV, most probable value; ng/L, nanograms per liter; \%, percent; $\mathrm{HCl}$, hydrochloric acid; MP1-MP5, mercuric nitrate standard diluted to target concentrations in $1 \% \mathrm{HCl}$ ]

\begin{tabular}{lcc}
\hline Solution Identifier & & $\begin{array}{c}\text { Total } \\
\text { Hg concentration } \\
\text { MPV } \\
\text { (ng/L) }\end{array}$ \\
\hline & 2009 & \\
\hline 1\% HCl BLANK & & 0.24 \\
MP1 & 6.10 \\
MP2 & 9.00 \\
MP3 & 15.2 \\
MP4 & 20.9 \\
\hline & & \\
\hline 1\% HCl BLANK & & 0.12 \\
MP1 & & 6.00 \\
MP2 & 9.20 \\
MP3 & 15.4 \\
MP4 & & 21.4 \\
\hline
\end{tabular}

acid $(\mathrm{HCl})$ blanks and mercuric nitrate spiked at five different concentrations in a 1-percent $\mathrm{HCl}$ matrix. These samples were identified as MP1, MP2, MP3, MP4, and MP5. The laboratories were instructed to analyze their samples as soon as they received them to promote accurate time representation of the data. All samples were single-blind samples, whereby the chemical analyst knows that the sample is a quality control sample but does not know the total $\mathrm{Hg}$ concentrations of the samples. The medians of all of the concentration values obtained from the participating laboratories were considered to be MPVs, which are listed in table 15 . Total $\mathrm{Hg}$ analysis data submitted by each laboratory were compared to MPVs for each solution, and differences between reported results and MPVs were plotted on control charts.

\section{Control Charts}

A visual comparison of interlaboratory differences between each laboratory's total Hg concentrations and MPVs are presented in the control charts shown in figures $22 A-22 B$. The warning limits are placed at plus or minus $2 f$-pseudosigma, and control limits are placed at plus or minus 3 $f$-pseudosigma from the zero difference line during the study period. The control chart for HAL in figure $22 A$ indicates negatively biased data compared to the MPVs during the study period with all results within statistical control limits during 2009-10.

\section{Interlaboratory Variability and Bias}

Methods for evaluation of the interlaboratory variability and bias for the MDN interlaboratory-comparison program are analogous to the evaluation of variability for the NTN interlaboratory-comparison program. The $f$-psig ratio was computed and expressed as a percentage for each laboratory using equation 2 , whereby an $f$-psig ratio larger than 100 percent indicates that the results provided by a laboratory had higher variability than the overall variability among the participating laboratories, and a ratio smaller than 100 percent indicates less variability than overall. The overall $f$-psig values were $0.52 \mathrm{ng} / \mathrm{L}$ and $0.55 \mathrm{ng} / \mathrm{L}$, for 2009 and 2010 respectively, over the concentration ranges shown in table 15. Interlaboratory bias was evaluated with the sign test for a median (Kanji, 1993). The arithmetic signs of the median differences indicated whether the reported total mercury analysis results were positively or negatively biased.

Results in table 16 indicate that HAL total Hg analyses had less variability than the overall variability with $f$-psig ratios of 96 percent and 57 percent for 2009 and 2010, respectively. The median MPV-minus-reported concentration difference for the HAL was the fifth largest among the participating laboratories during $2009(-0.21 \mathrm{ng} / \mathrm{L})$ and the third largest during $2010(-0.58 \mathrm{ng} / \mathrm{L})$ when evaluated on an absolute value basis. The negative bias observed for HAL during the study period was statistically significant $(\alpha=0.05)$ for both years. 
$A$

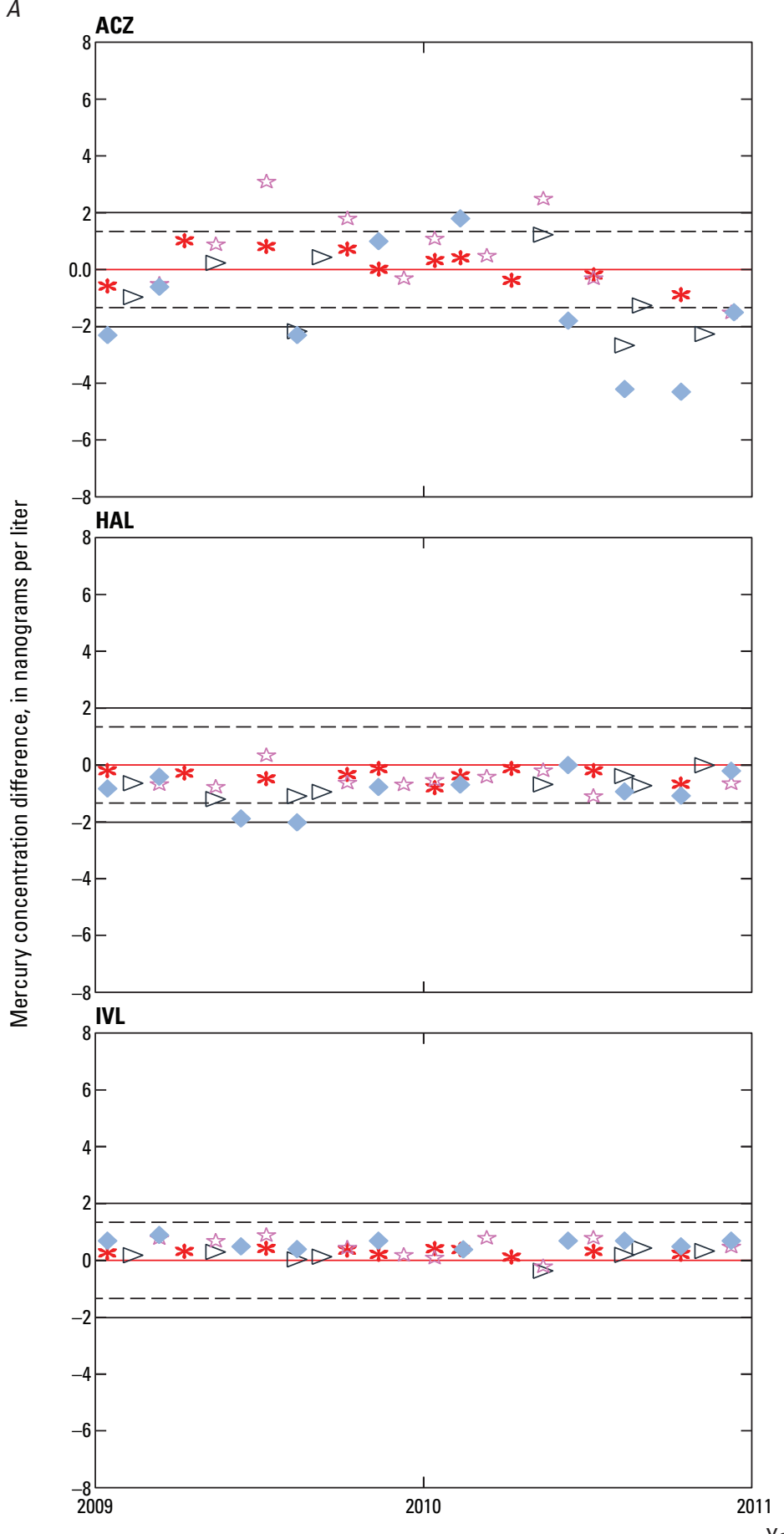

\section{EXPLANATION}

Control limit line -+3 and $-3 f$-pseudosigmas from zero difference Warning limit line -+2 and $-2 f$-pseudosigmas from zero difference Zero line

Solution-2009-2010 U.S. Geological Survey interlaboratory-comparison program, table 15 * MP1 is MP3

$\triangleright \quad$ MP2 $\diamond$ MP4

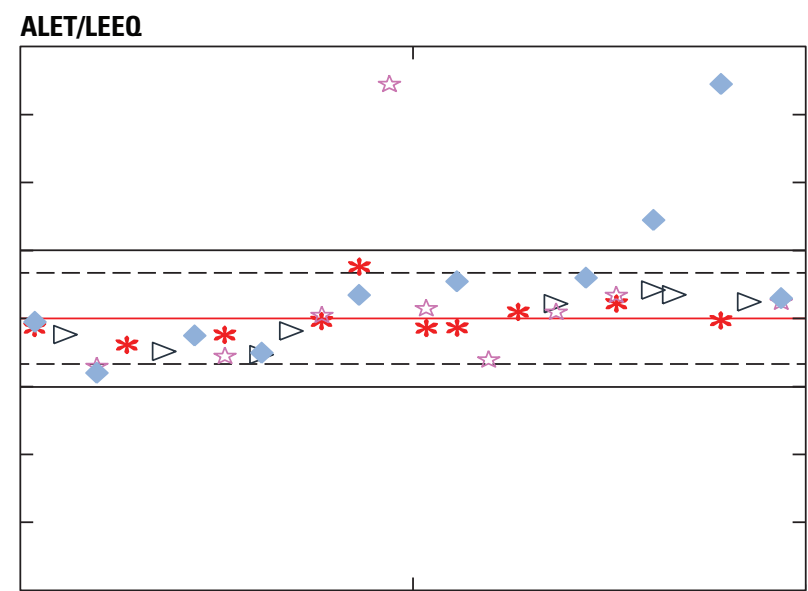

FRL

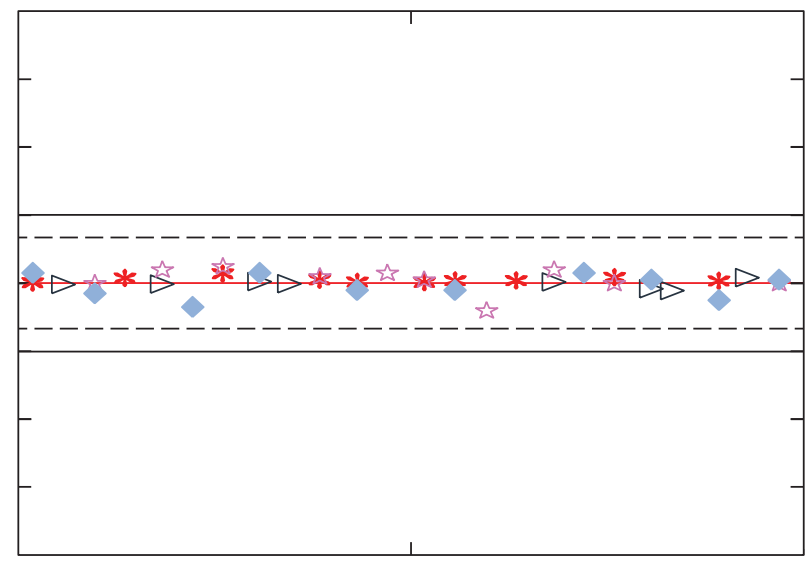

NLS

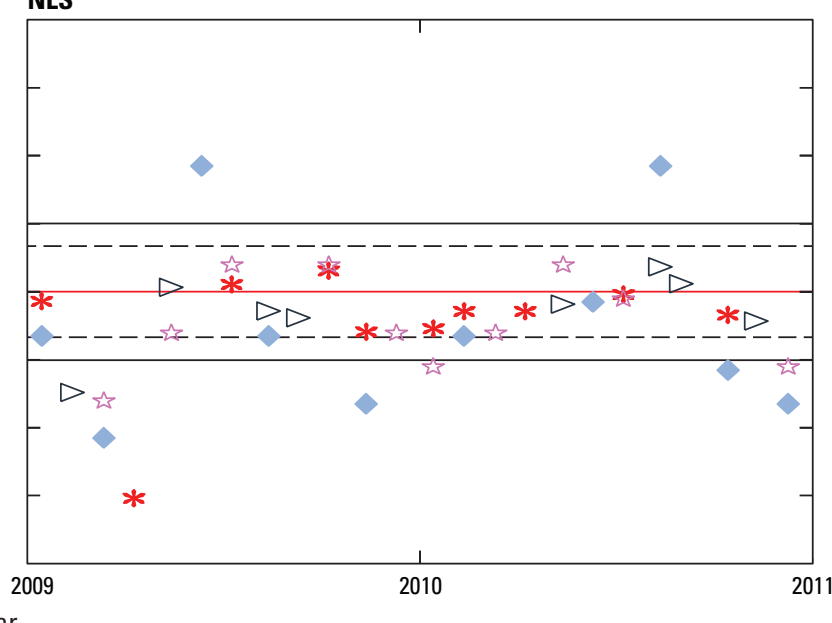

Laboratories:

ACZ-ACZ Laboratories, Steamboat Springs, Colorado

ALET/LEEQ -Atlantic/Quebec Laboratories of Environmental Testing, New Brunswick and Montreal, Canada

HAL-NADP Mercury Analytical Laboratory, Frontier Global Sciences, Inc., Seattle, Washington

FRL-Flett Research Limited, Winnipeg, Manitoba, Canada

IVL-Swedish Environmental Institute, Gotborg, Sweden

NLS—Northern Lake Service, Inc., Crandon, Wisconsin

Figure 22. A, Control charts for participating laboratories within the USGS interlaboratory-comparison program, Mercury Deposition Network, 2009-10. (NADP, National Atmospheric Deposition Program) 
B

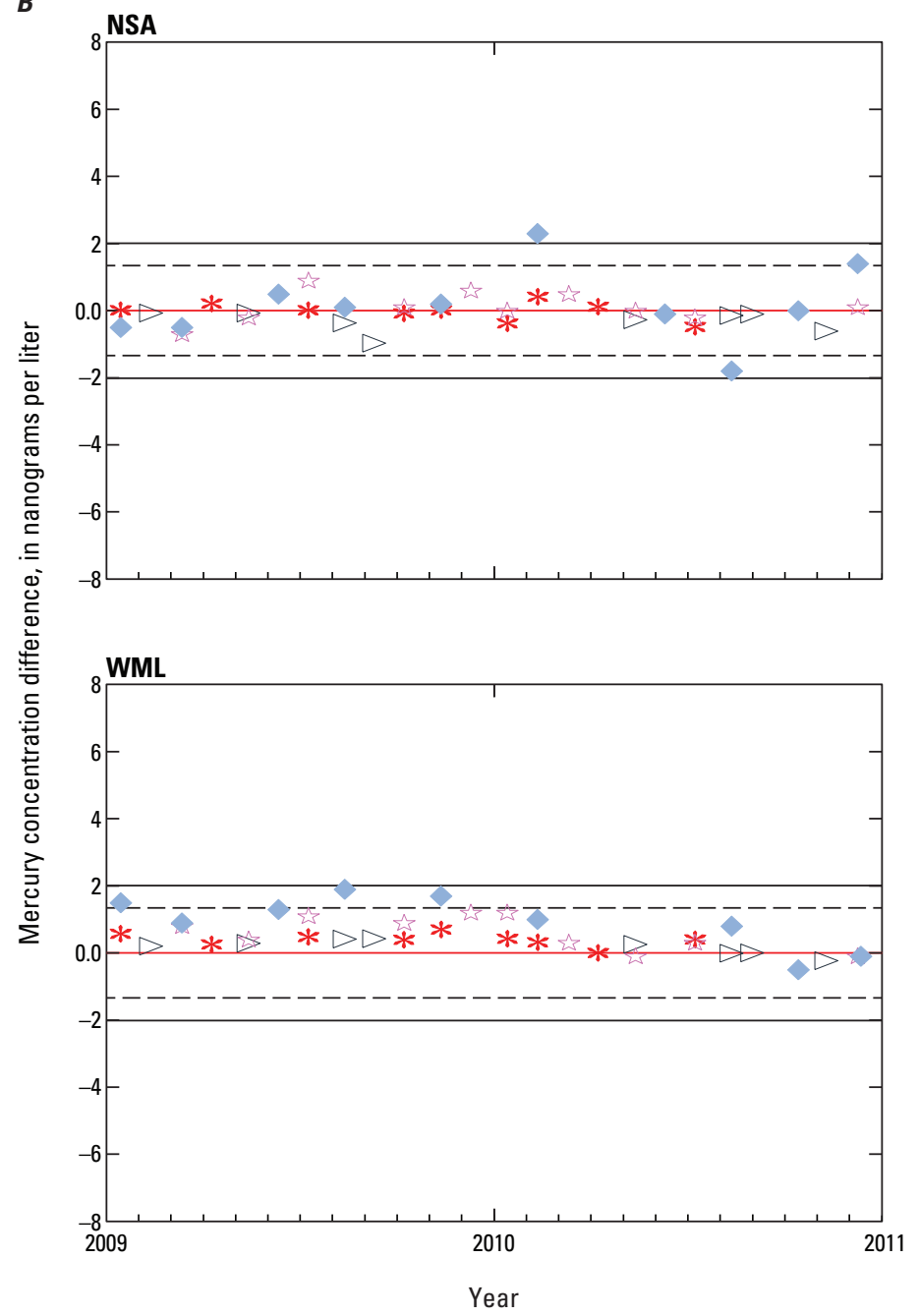

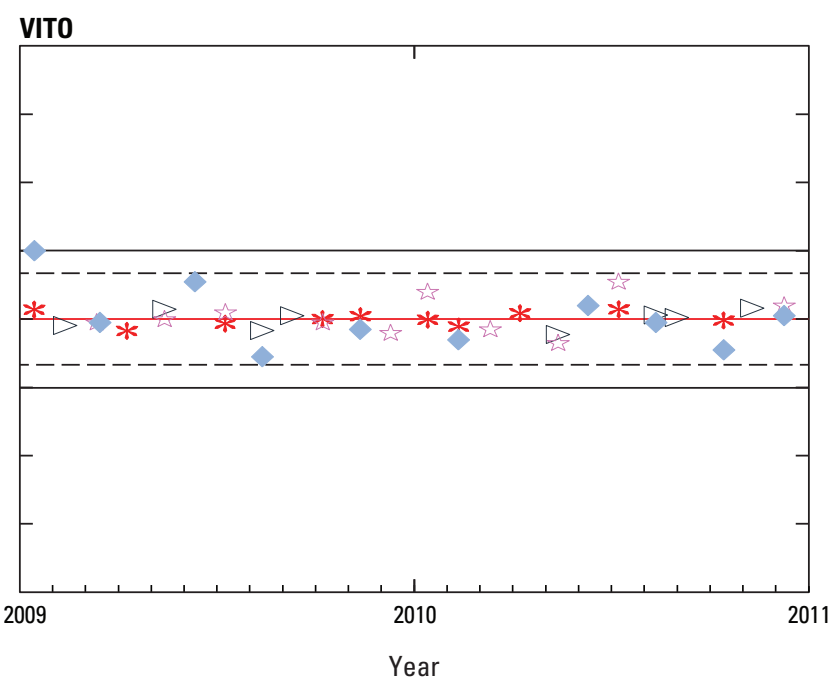

EXPLANATION

Control limit line -+3 and $-3 f$-pseudosigmas from zero difference Warning limit line -+2 and $-2 f$-pseudosigmas from zero difference Zero line

Solution-2009-2010 U.S. Geological Survey interlaboratorycomparison program, table 15

* MP1

$\triangleright \quad$ MP2

is $\quad \mathrm{MP} 3$

MP4

Laboratories:

NSA—North Shore Analytical, Inc., Duluth, Minnesota

VIT0_Flemish Technological Research Institute, Mol, Belgium

WML_USGS Wisconsin Mercury Laboratory, Middleton, Wisconsin

Figure 22. B, Control charts for USGS interlaboratory-comparison program for the Mercury Deposition Network, 2009-10. 
Table 16. Differences between reported mercury concentrations and most probable values for 2009-10 Mercury Deposition Network interlaboratory-comparison program samples.

[ng/L, nanograms per liter; shaded vales indicate statistically significant bias exceeding the method detection limit ( $\alpha=0.05$ ) (Kanji, 1993); \%, percent; ACZ, ACZ Laboratories, Inc.; ALET, Atlantic Laboratory of Environmental Testing; FRL, Flett Research, Ltd.; HAL, Mercury Analytical Laboratory, Frontier GeoSciences, Inc.; IVL, IVL-Swedish Environmental Institute; NLS, Northern Lake Service, Inc.; NSA, North Shore Analytical, Inc.; VITO, Flemish Institute for Technological Research; WML, U.S. Geological Survey Wisconsin Mercury Laboratory; LEEQ, Quebec Laboratory of Environmental Testing]

\begin{tabular}{|c|c|c|c|}
\hline Laboratory & $\begin{array}{c}\text { Median } \\
\text { difference }^{1} \\
\text { (ng/L) }\end{array}$ & $\begin{array}{c}\text { Sign } \\
\text { test } \\
p \text {-value }\end{array}$ & $\begin{array}{c}\text { f-psig } \\
\text { ratio } \\
(\%)\end{array}$ \\
\hline \multicolumn{4}{|c|}{2009} \\
\hline $\mathrm{ACZ}$ & 0.10 & 0.6291 & 212 \\
\hline ALET & -0.28 & 0.0963 & 141 \\
\hline FRL & 0.05 & 0.0352 & 58 \\
\hline HAL & -0.21 & 0.0003 & 96 \\
\hline IVL & 0.30 & 0.0005 & 59 \\
\hline NLS & -0.58 & 0.0213 & 388 \\
\hline NSA & 0.00 & 0.8036 & 43 \\
\hline VITO & 0.00 & 1.0000 & 77 \\
\hline WML & 0.47 & $<0.0001$ & 104 \\
\hline \multicolumn{4}{|c|}{2010} \\
\hline $\mathrm{ACZ}$ & -0.46 & 0.3593 & 365 \\
\hline ALET/LEEQ & 0.61 & 0.0213 & 81 \\
\hline FRL & 0.00 & 0.3877 & 18 \\
\hline HAL & -0.58 & 0.0003 & 57 \\
\hline IVL & 0.43 & 0.0023 & 76 \\
\hline NLS & -0.66 & 0.0192 & 215 \\
\hline NSA & -0.05 & 0.8036 & 73 \\
\hline VITO & 0.01 & 0.7905 & 51 \\
\hline WML & 0.24 & 0.4240 & 77 \\
\hline
\end{tabular}

${ }^{1}$ Median difference, median of differences between each laboratory's individual results and the most probable value (MPV), which is defined as the median of all results from all participating laboratories during 2009-10.

${ }^{2}$ Sign test $p$-value, probability of rejecting the null hypothesis: The true median of the differences between laboratory results and the most probable value is zero, when true.

${ }^{3} f$-psig ratio, ratio of each individual laboratory's $f$-pseudosigma to the overall $f$-pseudosigma ( $0.52 \mathrm{ng} / \mathrm{L}$ for 2009 and $0.55 \mathrm{ng} / \mathrm{L}$ for 2010), expressed as a percentage.
Table 17. Mercury Deposition Network interlaboratory comparison program results for blank samples, 2009-10.

[ng/L, nanograms per liter; < less than; Hg, mercury; ACZ, ACZ Laboratories, Inc.; ALET, Atlantic Laboratory of Environmental Testing; FRL, Flett Research, Ltd.; HAL, Mercury Analytical Laboratory, Frontier GeoSciences, Inc.; IVL, IVL-Swedish Environmental Institute; LEEQ, Quebec Laboratory of Environmental Testing; NLS, Northern Lake Service, Inc.; NSA, North Shore Analytical, Inc.; VITO, Flemish Institute for Technological Research; WML, U.S. Geological Survey Wisconsin Mercury Laboratory]

\begin{tabular}{lcc}
\hline Laboratory & $\mathbf{2 0 0 9}$ & $\mathbf{2 0 1 0}$ \\
\cline { 2 - 3 } & $\begin{array}{c}\text { Median total Hg } \\
\text { concentration } \\
\text { (ng/L) }\end{array}$ & $\begin{array}{c}\text { Median total Hg } \\
\text { concentration } \\
\text { (ng/L) }\end{array}$ \\
\hline ACZ & 0.3 & $<0.2$ \\
ALET & $<0.3$ & $<0.3$ \\
FRL & 0.14 & 0.12 \\
HAL & 0.2 & 0.17 \\
IVL & 0.12 & 0.09 \\
LEEQ & not participating & 0.06 \\
NLS & 0.28 & 0.22 \\
NSA & $<0.5$ & 0.08 \\
VITO & $<0.2$ & $<0.2$ \\
WML & 0.14 & 0.09 \\
\hline
\end{tabular}

\section{Results for MDN Interlaboratory-Comparison Program Blanks}

MDN interlaboratory-comparison results for 2009-10 blank samples shown in table 17. Minimum reporting levels (MRLs) vary between laboratories. MRLs were less than or equal to $0.5 \mathrm{ng} / \mathrm{L}$ during 2009-10. Median total $\mathrm{Hg}$ concentrations obtained for interlaboratory-comparison program blanks were less than $0.5 \mathrm{ng} / \mathrm{L}$ for both years. HAL blank results were similar to those from the other participating laboratories.

\section{Mercury Deposition Network Blind-Audit Program}

The MDN blind-audit program evaluated potential bias of HAL total mercury concentration data. For this program, USGS prepared and shipped deionized water blanks to 20 selected MDN sites, each accompanied by either a laboratory-created rain-gage chart or E-gage precipitation-depth value(s) to report to the NADP Program Office for the week. After a dry week, the site operators submitted the blind-audit samples and temporary, synthetic rain gage data to HAL in the place of the actual dry-week sample as if it were a real sample. The program is described in more detail by Latysh and Wetherbee (2007).

Percent recovery for each blind-audit $\mathrm{Hg}$ analysis was calculated by dividing the result obtained for the sample by the most probable value for $\mathrm{Hg}$ concentration in the solution 
as determined by the interlaboratory-comparison program (table 15) and multiplying by 100. In equation form,

$$
\left(\begin{array}{c}
\text { Percent recovery }= \\
\text { Hg concentration for blind }- \text { auditsample } \\
\text { Most probable Hg concentration for solution }
\end{array}\right) \times 100
$$

Fifteen MDN sites participated in the 2009 blind-audit program, and $16 \mathrm{MDN}$ sites participated in the 2010 blindaudit program. The median percent recovery for the study period was 96 percent in 2009 and 93 percent in 2010. Percent recovery was evaluated with respect to residence time between sample preparation and analysis and with respect to sample volume, but no relation between percent recovery and field residence time or sample volume was evident.

\section{Summary}

The U.S. Geological Survey (USGS) used three programs to provide external quality-assurance monitoring for the National Atmospheric Deposition Program/National Trends Network (NTN) and three programs to provide external quality-assurance monitoring for the NADP/Mercury Deposition Network (MDN) during 2009-10 (study period). The field-audit program assessed the effects of onsite exposure, sample handling, and shipping on the chemistry of NTN samples, and a system-blank program assessed the same effects for MDN. Two interlaboratorycomparison programs assessed the bias and variability of the chemical-analysis data from the Central Analytical Laboratory (CAL), Mercury (Hg) Analytical Laboratory (HAL), and 16 other participating laboratories for NTN and MDN programs combined. A co-located-sampler program was used to identify and quantify potential shifts in NADP data resulting from retrofit of network instrumentation with new electronic recording rain gages and precipitation collectors with optical sensors. A blind-audit program was implemented for the MDN to evaluate analytical bias in total mercury $(\mathrm{Hg})$ concentration data.

\section{National Trends Network}

\section{Contamination and Stability of NTN Samples}

Field-audit results for 2009-10 indicate that percentages of NTN sample concentrations less than the Network Maximum Contamination Limits (NMCLs) decreased from 2006-08 levels for all analytes except ammonium and nitrate. During 2008-10, ammonium and nitrate losses from NTN samples were greater than 2006-08 losses by approximately 0.40 and $0.01 \mathrm{mg} / \mathrm{L}$, respectively. Hydrogen-ion loss also increased slightly during 2008-10.

\section{Laboratory Analysis of NTN Samples}

Sodium and sulfate concentrations were slightly biased high, and nitrate concentrations were slightly biased low for
CAL interlaboratory-comparison program results during 2009, but no bias was indicated during 2010. Results for the CAL had the lowest overall variability of the eight participating laboratories during 2009-10; CAL results also indicated no detections of analytes greater than the MDLs for deionized water blanks during 2009-10.

Control charts for CAL show more values outside the statistical control limits than typically observed in the recent past, especially for calcium, magnesium, sodium, and potassium results for natural precipitation samples. However, CAL data were within statistical control during at least 90 percent of the study period. High variability among all of the participating laboratories for ammonium and nitrate results for natural samples might indicate instability of the nitrogen species that varied among the samples for each laboratory.

\section{Evaluation of Rain Gages and Precipitation- Collectors}

The USGS compared the Belfort Model 5-780 rain gages with the ETI Noah-IV and and OTT Pluvio-N rain gages during 2007-09. During water years 2007-09, median weekly precipitation depths measured with Noah-IV rain gages were 5.7 to 8.0 percent greater than co-located Belfort 5-780 rain gage depths. The OTT Pluvio-N rain gage measured approximately 4.1 percent greater precipitation depth than the Belfort rain gage at IL11 during 2009. Identical Noah-IV and OTT Pluvio-N gages were compared at co-located sites during 2010. Co-located, identical Noah-IV gages recorded precipitaiton depths within 0.6 to 2.2 absolute percent error during 2010. Co-located, identical OTT Pluvio-N gages recorded precipitation depths within 1.7 absolute percent error at IN26 during 2010. Bias between identical, paired rain gages with respect to precipitation phase (frozen versus liquid) was statistically significant, but the magnitudes of the measured biases were small enough to be practically negligible.

Co-located Aerochem Metrics model 301 (ACM) and $\mathrm{N}-\mathrm{CON}$ NTN bucket precipitation collectors were operated at VT99/99VT and IL11/96IL during 2009-10. The N-CON collectors were mechanically more reliable than the ACM collectors, especially during winter conditions. The N-CON collectors cycled more and exposed the buckets to the atmosphere more frequently than the ACM collectors. This prompted the conclusion that N-CON collectors obtain larger sample volumes and generally higher analyte concentrations and annual deposition estimates, except for hydrogen ion. Median weekly concentration differences between the ACM and N-CON were between 0.003 to $0.008 \mathrm{mg} / \mathrm{L}$ for magnesium, sodium, potassium, and chloride, and between 0.017 to $0.110 \mathrm{mg} / \mathrm{L}$ for calcium, ammonium, nitrate, and sulfate. The N-CON collected significantly ( $\alpha=0.05,95$ percent confidence) higher concentrations than the ACM. Significant differences $(\alpha=0.05)$ in precipitation-type bias were also observed for the N-CON collector for all analytes except potassium, ammonium, and hydrogen ion. 


\section{Mercury Deposition Network}

\section{Contamination and Stability of MDN Samples}

Results of the 2009-10 USGS system-blank program were used to compute a NMCL for total $\mathrm{Hg}$ of $1.545 \mathrm{ng} / \mathrm{L}$ for the study period. In other words, the maximum contamination in MDN samples during 2009-10 was not greater than $1.545 \mathrm{ng} / \mathrm{L}$ with 90-percent confidence, and also, no more than 10 percent of the MDN samples had contamination concentrations exceeding $1.545 \mathrm{ng} / \mathrm{L}$ with 90 -percent confidence. This level is approximately equal to the 2 nd percentile of all MDN weekly Hg concentrations. Approximately 40 percent of the $\mathrm{Hg}$ contamination concentrations were less than the MRL (0.15 ng/L). The 90-percent UCLs on the 3-yr moving average 90th percentiles of $\mathrm{Hg}$ contamination mass per MDN sample increased steadily from 0.095 nanograms per sample (ng/sample) during 2004-06 to $0.325 \mathrm{ng} /$ sample during 2007-09 and 2008-10.

\section{Laboratory Analysis of MDN Samples}

Total $\mathrm{Hg}$ analyses done at HAL had less variability than the overall interlaboratory variability, with $f$-psig ratios of 96 percent and 57 percent for 2009 and 2010, respectively. The median MPV-minus-reported concentration difference for the HAL was the fifth largest among the participating laboratories during $2009(-0.21 \mathrm{ng} / \mathrm{L})$ and the third largest during $2010(-0.58 \mathrm{ng} / \mathrm{L})$ when evaluated on an absolutevalue basis. The negative bias observed for HAL during the study period was statistically significant $(\alpha=0.05)$ for both years. Median total $\mathrm{Hg}$ concentrations obtained for interlaboratory-comparison program blanks were less than $0.5 \mathrm{ng} / \mathrm{L}$ for both years. HAL blank results were similar to those from the other participating laboratories.

Fifteen MDN sites participated in the 2009 blind-audit program, and $16 \mathrm{MDN}$ sites participated in the 2010 blindaudit program. The median percent recovery for the study period was 96 percent in 2009 and 93 percent in 2010. No relation between percent recovery and field-residence time or sample volume was evident.

\section{References Cited}

Aikawa, M. and Hiraki, T., 2009, Washout/rainout contribution in wet deposition estimated by $0.5 \mathrm{~mm}$ precipitation sampling/analysis, Atmospheric Environment, no. 43, p 4935-4939.

Berthouex, P.M., and Brown, L.C., 1995, Statistics for environmental engineers: Boca Raton, Fla., CRC Press, Inc., $489 \mathrm{p}$.

Chambers, J.M., Cleveland, W.S., Kleiner, B., and Tukey, P.A., 1983, Graphical methods for data analysis: Boston, Mass., Duxbury Press, 395 p.
Colin, J.L., Jaffrezo, J.L., Pinart, J., and Roulette-Cadene, S., 1987, Sequential sampling of snow in a rural area Experimentation and identification of the acidifying agents: Atmospheric Environment, v. 21, no. 5, p 1147-1157.

Dossett, S.R., and Bowersox, V.C., 1999, National Trends Network site operation manual: Champaign, Ill., National

Atmospheric Deposition Program Office at the Illinois State Water Survey, NADP Manual 1999-01, 94 p., accessed December 27, 2007 at http://nadp.sws.uiuc.edu/lib/manuals/ opman.pdf.

Fishman, M.J., Schroder, L.J., and Schockey, M.W., 1986, Evaluation of methods for preservation of water samples for nutrient analysis: International Journal of Environmental Studies, v. 26, p. 231-238.

Gibbons, R.D., and Coleman, D.E., 2001, Statistical methods for detection and quantification of environmental contamination: New York, John Wiley \& Sons, 87 p.

Gordon, J.D., 1999, External quality-assurance results for the National Atmospheric Deposition Program/National Trends Network, 1995-96: U.S. Geological Survey WaterResources Investigations Report 99-4072, 69 p.

Hahn, G.J., and Meeker, W.Q., 1991, Statistical intervalsA guide for practitioners: New York, John Wiley \& Sons, $392 \mathrm{p}$.

Helsel, D.R., 2012, Statistics for censored environmental data using Minitab and R ( $2 \mathrm{~d}$ ed.): Hoboken, New Jersey, John Wiley and Sons, p 70-77.

Helsel, D.R., and Hirsch, R.M., 1992, Statistical methods in water resources: New York, Elsevier Science Publishing Company, Inc., $522 \mathrm{p}$.

Hoaglin, D.C., Mosteller, F., and Tukey, J.W., 1983, Understanding robust and exploratory data analysis: New York, John Wiley and Sons, $432 \mathrm{p}$.

Hollander, M., and Wolfe, D.A, 1999, Nonparametric statistical methods (2d ed.): New York, John Wiley \& Sons, 787 p.

Kanji, H.K., 1993, 100 statistical tests: Newbury Park, Calif, Sage Publications Inc., 216 p.

Latysh, N.E., and Wetherbee, G.A., 2005, External quality assurance programs and procedures managed by the U.S. Geological Survey in support of the National Atmospheric Deposition Program/National Trends Network: U.S. Geological Survey Open-File Report 2005-1024, 66 p.

Latysh, N.E., and Wetherbee, G.A., 2007, External quality assurance programs and procedures managed by the U.S. Geological Survey in support of the National Atmospheric Deposition Program/Mercury Deposition Network: U.S. Geological Survey Open-File Report 2007-1170, 33 p. 
Lynch, J., DeWalle, D., and Horner, K., 1989, Impact of NADP/NTN sampling protocols on winter storm estimates of wet deposition in Central Pennsylvania: Environmental Protection Agency Report ER8905, University Park, Penn., Penn State, Environmental Resources Research Institute, $8 \mathrm{p}$.

National Atmospheric Deposition Program, 2009, Network Operations Subcommittee (NOS)/Data Management and Analysis Subcommittee (DMAS) meeting minutes, Tuesday, April 28, 2009: Pensacola, Florida, National Atmospheric Deposition Program, 8 p., accessed July 12, 2013 at http://nadp.isws.illinois.edu/committees/minutes.aspx.

National Atmospheric Deposition Program, 2011, National Atmospheric Deposition Program 2010 Annual Summary: University of Illinois at Urbana-Champaign, Ill., Illinois State Water Survey, NADP Data Report 2011-01, 23 p.

Nilles, M.A., 2001, Changes in the chemistry of precipitation in the United States, 1981-1998: Water, Air, \& Soil Pollution, v. 130 , no. 1, p. 409-414.

Nilles, M.A., See, R.B., Willoughby, T.C., and Gordon, J.D., 1991, Variability in wet atmospheric deposition data determined with co-located samplers: U.S. Geological Survey Water-Resources Investigations Report 914143, 30 p.

SAS Institute, Inc., 2001, SAS STAT users guide, version 8.2: Cary, N.C., SAS Institute, Inc.

Schroder, L.J. and Hedley, A.G., 1986, Variation in precipitation quality during a 40-hour snowstorm in an urban environment-Denver, Colorado: International Journal of Environmental Studies, v. 28, pp 131-138.

Tchobanoglous, G., and Schroeder, E.D., 1987, Water quality: Reading, Mass., Addison-Wesley Publishing Company, Inc., $768 \mathrm{p}$.

U.S. Environmental Protection Agency, 2002, Method 1631 revision E, mercury in water by oxidation, purge and trap, and cold vapor atomic fluorescence spectrometry: Washington D.C., U.S. Environmental Protection Agency, Office of Water, EPA-821-R-02-019, 38 p., accessed July 12, 2013 at http://water.epa.gov/scitech/methods/cwa/metals/mercury/ index.cfm/2007_07_10_methods_method_mercury_1631.pdf.

Wetherbee, G.A., Latysh, N.E., and Gordon, J.D., 2005a, Spatial and temporal variability of the overall error of National Atmospheric Deposition Program measurements determined by the USGS co-located-sampler program, water years 1989-2001: Environmental Pollution, v. 135, p. $407-418$.

Wetherbee, G.A., Latysh, N.E., and Burke, K.P., 2005b, External quality assurance results for the National Atmospheric Deposition Program/National Trends Network, 2002-03: U.S. Geological Survey Scientific Investigations Report 2005-5061, $59 \mathrm{p}$.
Wetherbee, G.A., Latysh, N.E., and Greene, S.M., 2006, External quality assurance results for the National Atmospheric Deposition Program/National Trends Network, 2004: U.S. Geological Survey Scientific Investigations Report 2005-5067, 52 p.

Wetherbee, G.A., Latysh, N.E., Greene, S.M., and Chesney, T., 2009, U.S. Geological Survey External Quality-Assurance Program Results reported to the National Atmospheric

Deposition Program/National Trends Network and Mercury Deposition Network for 2005-06: Champaign, Ill., Illinois State Water Survey, NADP Quality Assurance Report 200901, Illinois State Water Survey Data/Case Study 2009-01, 54 p. [PDF File (5.08 MB) http:/nadp.sws.uiuc.edu/lib/qa/ nadp_qa0506.pdf].

Wetherbee, G.A., Latysh, N.E., Greene, S.M., and Chesney, T., 2010, U.S. Geological Survey External Quality-Assurance Program Results reported to the National Atmospheric Deposition Program/National Trends Network and Mercury Deposition Network for 2007-08: Champaign, Ill., Illinois State Water Survey, NADP Quality Assurance Report 201001, Illinois State Water Survey Data/Miscellaneous Publication 190, 82 p. [PDF File (7.65 MB) http:/nadp.sws.uiuc. edu/lib/qa/nadp_qa0708.pdf].

Wetherbee, G.A., Latysh, N.E., and Gordon, J.D., 2004, External quality assurance results for the National Atmospheric Deposition Program/National Trends Network, 2000-01: U.S. Geological Survey Scientific Investigations Report 2004-5034, 59 p.

Wetherbee, G.A., and Rhodes, M.F., 2013, Effects of equipment performance on data quality from national atmospheric deposition program/national trends network and mercury deposition network: U.S. Geological Survey OpenFile Report 2013-1031, 53 p.

Wilde, F.D., Radtke, D.B., Gibs, J., and Iwatsubo, R.T., 1998, Selection of equipment for water sampling, in National field manual for the collection of water-quality data-Collection of water samples: U.S. Geological Survey Techniques of Water Resources Investigations, book 9, chap. A2, p. 57.

Publishing support provided by:

Denver Publishing Service Center

For more information concerning this publication, contact:

Chief, USGS Branch of Quality Systems

Box 25046, Mail Stop 401

Denver, CO 80225

(303) 236-1835

Or visit the Branch of Quality Systems Web site at: http://bqs.usgs.gov/ 


\section{$\frac{\mathbb{2}}{3}$}

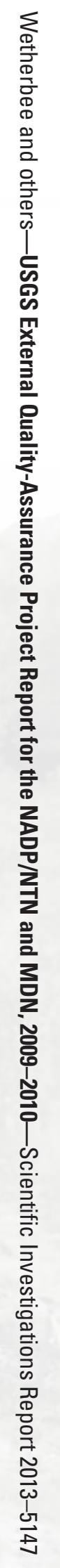

